\title{
The impact of pain-related fear and hypermobility on physical functioning in adolescents with chronic musculoskeletal pain
}

Citation for published version (APA):

van Meulenbroek, T. (2021). The impact of pain-related fear and hypermobility on physical functioning in adolescents with chronic musculoskeletal pain. [Doctoral Thesis, Maastricht University]. Maastricht University. https://doi.org/10.26481/dis.20210423tm

Document status and date:

Published: 01/01/2021

DOI:

10.26481/dis.20210423tm

Document Version:

Publisher's PDF, also known as Version of record

Please check the document version of this publication:

- A submitted manuscript is the version of the article upon submission and before peer-review. There can be important differences between the submitted version and the official published version of record.

People interested in the research are advised to contact the author for the final version of the publication, or visit the DOI to the publisher's website.

- The final author version and the galley proof are versions of the publication after peer review.

- The final published version features the final layout of the paper including the volume, issue and page numbers.

Link to publication

\footnotetext{
General rights rights.

- You may freely distribute the URL identifying the publication in the public portal. please follow below link for the End User Agreement:

www.umlib.nl/taverne-license

Take down policy

If you believe that this document breaches copyright please contact us at:

repository@maastrichtuniversity.nl

providing details and we will investigate your claim.
}

Copyright and moral rights for the publications made accessible in the public portal are retained by the authors and/or other copyright owners and it is a condition of accessing publications that users recognise and abide by the legal requirements associated with these

- Users may download and print one copy of any publication from the public portal for the purpose of private study or research.

- You may not further distribute the material or use it for any profit-making activity or commercial gain

If the publication is distributed under the terms of Article $25 \mathrm{fa}$ of the Dutch Copyright Act, indicated by the "Taverne" license above, 
The impact of pain-related fear and hypermobility on physical functioning in adolescents with chronic musculoskeletal pain

Thijs van Meulenbroek 
ISBN: 978-94-6361-483-2

Cover design: Marly Thissen - Peters

Layout: $\quad$ Tiny Wouters - Lenssen

Printed by: Prinstudio INDRUK

(C) 2021 T. van Meulenbroek, Urmond, the Netherlands

All rights reserved. No part of this thesis may be reproduced or transmitted in any form or by any means, electronical or mechanical, including photocopy, recording or any other information storage or retrieval system, without written permission of the copyright owner.

The printing of this thesis was financially supported by de Vereniging van Ehlers-Danlos patiënten (VED). 


\title{
The impact of pain-related fear and hypermobility on physical functioning in adolescents with chronic musculoskeletal pain
}

\author{
Dissertation \\ to obtain the degree of Doctor at the Maastricht University, \\ on the authority of the Rector Magnificus, Prof. dr. R.M. Letschert \\ in accordance with the decision of the Board of Deans, \\ to be defended in public on Friday, April 23, 2021, at 14:00 hours
}

by

Thijs van Meulenbroek

Born on the $11^{\text {th }}$ of February 1986 in Heerlen, the Netherlands 


\section{Promotores}

Prof. Dr. J.A. Verbunt

Prof. Dr. R.H.H. Engelbert (Universiteit van Amsterdam, Amsterdam)

\section{Copromotor}

Dr. I.P.J. Huijnen

\section{Beoordelingscommissie}

Prof. Dr. R.J.E.M. Smeets (voorzitter)

Prof. Dr. R.A. de Bie

Prof. Dr. A.I. Buizer (Vrije Universiteit, Amsterdam)

Dr. L. Rombaut (Universiteit Gent, Gent, België)

Prof. Dr. J.W.S. Vlaeyen

The research presented in this thesis was performed at the department of Rehabilitation Medicine (Care and Public Health Research Institute CAPHRI), Maastricht University, the Netherlands. This project was funded by Adelante, Centre of Expertise in Rehabilitation and Audiology Hoensbroek; Fonds Nuts Ohra and Stichting Vooruit. 
"Stoer sterk

omringd alleen

knoestig fragiel

ontredderd geknapt"

"Nat intens koud

kale takken drukkende knopjes

warme zonnestralen

bewegende groei"

"Gesteund gewerkt

mooie nerf

stevig zelfstandig

volwaardig bestaan"

Marly Thissen - Peters, 2021 



\section{Table of contents}

$\begin{array}{lll}\text { Chapter } 1 \text { General introduction } & 9\end{array}$

Chapter 2 Exploring the underlying mechanism of pain-related disability in

hypermobile adolescents with chronic musculoskeletal pain

Chapter 3 Pain-related fear and its disabling impact in hypermobile adolescents with chronic musculoskeletal pain

Chapter 4 Generalized joint hypermobility and perceived harmfulness in healthy adolescents; impact on muscle strength, motor performance, and physical activity level

Chapter 5 Are chronic musculoskeletal pain and generalized joint hypermobility disabling contributors to physical functioning?

Chapter 6 Multidisciplinary treatment for hypermobile adolescents with chronic musculoskeletal pain

Chapter 7 General discussion

Summary

Samenvatting

Impact paragraph

Dankwoord

About the author 



\section{Chapter 1}

General introduction 



\section{General introduction}

Pain is one of the most common human experiences and the ability of feeling pain is vital to protect the human body for injury, harm or tissue damage. ${ }^{1}$ Pain serves as an adaptive function in the acute phase, since it enhances the body to promote healing with a defensive response to minimize contact with damaging stimuli or by creating a situation that discourages physical contact and movement. ${ }^{2}$ The definition of pain according to the International Association for the Study of Pain (IASP) is "an unpleasant sensory and emotional experience associated with, or resembling that associated with, actual or potential tissue damage". ${ }^{3}$ However, when pain persists after the normal healing time and has no acute warning function of physiological nociception, it is defined as chronic pain. ${ }^{4}$ According to the International Classification of Diseases (ICD-11) chronic pain may be divided into primary, a health condition in its own right, vs secondary, in which pain may be considered a symptom of some other underlying disease. ${ }^{5}$ Chronic primary pain is defined as pain that persists or reoccurs for more than 3 months and characterized by disability and emotional distress. ${ }^{6}$ People from all ages, from babies to elderly, may experience chronic pain. Globally, it has been estimated that $20 \%$ of the adults suffer from chronic pain and that another $10 \%$ is annually diagnosed with chronic pain. ${ }^{7,8}$ In recent years, chronic pain in children and adolescents has gained attention. In this group, pain is also a serious health condition with a potential large impact on daily functioning, development, and participation in society. However, the children and adolescent population has important different characteristics as compared to the adult population, such as growing and developing body and mind with different stages (e.g. puberty) and the influence of parental and family factors during the varying developmental phases (infancy, toddler, childhood, adolescence, and young adults). ${ }^{9}$ Based on that, adolescents with chronic pain have important characteristics that highlight the need for specialised care. ${ }^{10}$ Therefore, this dissertation focusses on the adolescent population with an age range of 12-21 years. ${ }^{11}$ Based on location chronic pain can be divided in subgroups. Along with abdominal pain and headache, chronic musculoskeletal pain (CMP) is one of the most frequently reported pain complaints in adolescents. ${ }^{12,13}$ CMP is defined as persistent or recurrent pain directly affecting bones, joints, muscles or related soft tissue. ${ }^{4}$ Although a specific somatic cause of CMP could be identified, most CMP is defined as non-specific. ${ }^{14}$ Because in these cases, mostly no solution is available to cure CMP, a biopsychosocial approach in the management of CMP is recommended. In this approach, the complex relation between the pain experience and its impact on daily life and participation in society is unravelled and focusses on the complex interaction between physiological, psychological, and social factors that influence CMP and accompanying disability. ${ }^{14-16}$ 
Two important factors which might contribute to understand the complexity and explain the disabling impact of CMP are the presence of pain-related fear and generalized joint hypermobility (GJH). In paediatric chronic pain patients, pain-related fear has shown to be a predictor of pain-related disability ${ }^{17,18}$ and physical activity limitations. ${ }^{19}$ Two longitudinal cohort studies showed that during adolescence, GJH represents a risk factor for the development of CMP. ${ }^{20,21}$ In addition, the prevalence of GJH in children with CMP is striking (40-48\%). ${ }^{22,23}$ The aim of this dissertation is to further unravel the role of painrelated fear and GJH on physical functioning in adolescents with CMP. Physical functioning is part of the multi-dimensional concept functional status, defined as a patient-oriented health outcome which contains aspects of daily functioning, including physical, psychological, and social factors. ${ }^{24}$ Better understanding might lead to better tailored and personalised interventions.

\section{Epidemiology and impact of CMP in childhood and adolescence}

In paediatric CMP prevalence rates ranged from $4-40 \%$ in children and adolescents and the prevalence increased with age, with a peak in CMP at age of 16 years. Furthermore, CMP is more common in girls, than in boys. ${ }^{25}$ Longitudinal studies showed that CMP is not self-limiting; persistence rates of pain complaints between $30 \%$ and $64 \%$ have been reported in schoolchildren aged 10-12 years after 4 years follow-up. ${ }^{12,26}$ In addition, another study even showed a persistence rate of pain of $20 \%$ after 13 years from young schoolchildren into early adulthood. ${ }^{27}$ Furthermore, in approximately $40 \%$ of children and adolescents with CMP, pain has a disabling impact. ${ }^{28,29} \mathrm{CMP}$ has a negative impact on physical and psychological functioning, interferes with school attendance, performance in social activities, and family engagement, reducing the child's and adolescent's quality of life. ${ }^{15,29-31}$ In addition, in children with CMP increased rates of use of health care services, such as seeking for medical attention, referral for diagnostics, and treatments are reported. ${ }^{32,33}$ Furthermore, studies estimated direct costs in health care expenditure as well as indirect costs such as productivity loss of parents due to work absence of $£ 8000$ in the UK ( $€ 8880$ when converted to 2020 EUR) and \$11.787 in the US (€9901 when converted to 2020 EUR) per adolescent per year. Based on that the economic burden of CMP in adolescents on society has been estimated to be substantial. ${ }^{34,35}$

\section{Generalized joint hypermobility}

Generalized joint hypermobility $(\mathrm{GJH})$ is defined as an increased range of motion in most of the joints. ${ }^{36}$ The prevalence of GJH in children and adolescents varies from 2-55\%, depending on ethnicity, gender, age, and the tests and criteria applied..$^{37,38}$ The Beighton 
score (BS) (Figure 1.1) is widely used in clinical practice and is considered to be the golden standard for assessing the presence of $\mathrm{GJH}^{39}$ Although the $\mathrm{BS}$ is a reliable assessment, the validity for identifying GJH is questionable. ${ }^{40,41}$ Throughout the years different cut-off points have been used for the BS. Especially in children and adolescents there was no consensus. ${ }^{42}$ However, in 2017 a consensus statement was published with unified criteria for the use of BS and recommended in adults a cut-off point of 5 out of 9 , while in children and adolescents the cut-off point is 6 out of $9 .^{42}$

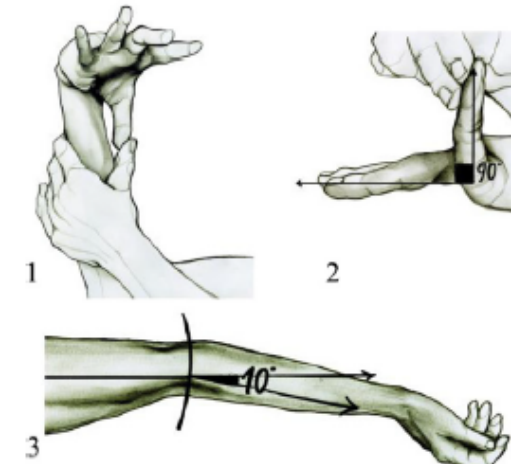

Figure 1.1 The functional tests of the Beighton score. Adapted from Folci and Capsoni, $2016^{43}$, with permission.

Earlier studies indicated that adolescents with GJH had decreased daily physical activities ${ }^{44}$ and lower muscle strength. ${ }^{45,46}$ In addition, adolescents and young adults with GJH participated less during sports and outdoor activities, but spent more time on walking and cycling during leisure time. ${ }^{45}$ However, other studies could not confirm the reduced muscle strength ${ }^{47}$ and altered physical activity level ${ }^{48}$ in adolescents with GJH compared to non-hypermobile controls. Thus, it remains unclear what the consequences are of having GJH on physical functioning and societal participation. These contradicting results are in line with the wide variety of presentation in daily life functioning of individuals with $\mathrm{GJH}$. For many individuals with $\mathrm{GJH}$, flexible joints deliver beneficial effects in the performance of sport activities, such as dancing or gymnastics. ${ }^{49}$ Also performing artists like musicians, who require especially joint flexibility in their hands to maximize the control of their instruments, have advantage of $\mathrm{GJH} .{ }^{50}$ However, for others, GJH can be disabling since it is related to various symptoms such as CMP. 


\section{Symptomatic generalized joint hypermobility}

In 2017 a consensus statement with new nosology according to unified criteria is published and GJH related to symptoms such as CMP, joint instability, disturbed proprioception, and soft tissue injuries is now referred to as generalized hypermobility spectrum disorder (G-HSD) ${ }^{51}$, the former joint hypermobility syndrome. ${ }^{52}$ In addition, GJH is also frequently observed in conditions belonging to the group of hereditary diseases of the connective tissue (HDCT), such as Ehlers-Danlos syndrome, osteogenesis imperfecta, and Marfan syndrome. ${ }^{53}$ These rare serious connective tissues diseases have specific genetic profiles and clinical characteristics. However, within the Ehlers-Danlos syndrome spectrum there is a hypermobility subtype; characterised by GJH and CMP, in combination with a positive family history, and systematic manifestations of a more generalized connective tissue disorder, such as skin laxity. However, this subtype is also lacking a specific genetic profile and is known as hypermobile Ehlers-Danlos syndrome (hEDS) according to the new nosology ${ }^{54}$, the former hypermobility type of Ehlers-Danlos syndrome/Ehlers-Danlos syndrome type $11{ }^{55}$ Thus, G-HSD and hEDS are both symptomatic GJH-related disorders lacking a specific genetic profile with an overlap in the clinical presentation and in this dissertation they are referred to as G-HSD/hEDS (Figure 1.2). In addition, many studies referred to in this dissertation are conducted before the new diagnostic criteria and used the old nomenclature. Therefore, it should be noted that the consequences of these new criteria for patient demographics and disease characteristics are unknown yet. However, we still decided to refer to G-HSD/hEDS in our studies who were conducted after the published consensus statement.

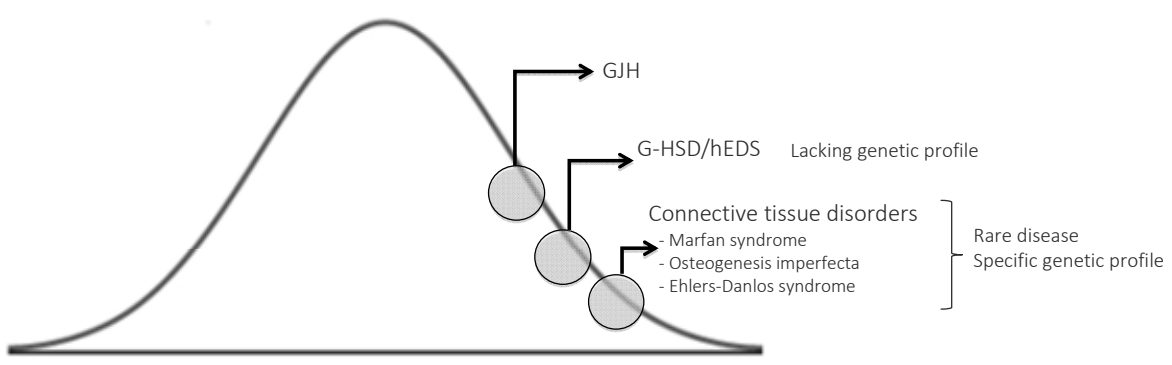

Figure 1.2 The hypermobility spectrum. Adapted from Scheper, $2019^{56}$, with permission.

Abbreviations: GJH = generalized joint hypermobility; G-HSD = generalized hypermobility spectrum disorder; hEDS = hypermobile Ehlers-Danlos syndrome. 
Common physical symptoms in children and adolescents with G-HSD/hEDS are muscle weakness ${ }^{57}$, decreased exercise tolerance ${ }^{58,59}$, impaired proprioception ${ }^{57}$, and reduced balance ${ }^{60}$ compared to pain-free children and adolescents. In addition, children and adolescents with G-HSD/hEDS reported decreased levels of participation in activities ${ }^{39,60}$, difficulties in gross motor skills ${ }^{60}$, and impaired gait. ${ }^{61}$ Furthermore, multi-systemic complaints (including incontinence, constipation, autonomic and cardiac dysfunction) ${ }^{62,63}$, sleep disturbance ${ }^{64}$, and fatigue ${ }^{65}$ are prevalent. Recently, the presence of generalized hyperalgesia is reported in individuals with G-HSD/hEDS ${ }^{6,67}$ suggesting hypersensitivity of the central nervous system. In addition, psychological involvement of pain-related fear ${ }^{68,69}$, more generalized anxiety ${ }^{70,71}$, and increased incidence of psychiatric conditions, such as panic disorders and depression ${ }^{72}$ are also present in individuals with G-HSD/hEDS. Thus, children and adolescents with G-HSD/hEDS may have a large variability of complaints and severity leading to disabilities in daily living, physical, psychological, and social functioning.

\section{Treatment of adolescent with CMP and GJH}

The current evidence-base treatment and management of children and adolescents with G-HSD/hEDS is limited in size and quality. ${ }^{73,74}$ Several studies showed that monodisciplinary physical therapy leads to significant improvements in disability and pain intensity. ${ }^{75-77}$ In addition, a physiotherapist-prescribed exercise programme for children with G-HSD/hEDS and hypermobile knees led to improvements in knee muscle strength, pain reduction, and increased self-esteem. ${ }^{78}$ Other studies evaluating multidisciplinary treatments combining physical and cognitive rehabilitation found improvements in disability, muscle strength, and psychological factors, such as kinesiophobia and catastrophizing thoughts. ${ }^{79,80}$ In addition, a randomised controlled trial compared multidisciplinary treatment (physiotherapy and occupational therapy) with standard care. However, no additional significant benefit was seen from the intervention compared to the standard care. Both groups showed improvements in pain intensity by the child, parents' proxy reports of pain intensity, coordination, and grip strength. $^{81}$

However, in most of the interventions, there are several different treatments with a wide variety of elements lacking a specific theoretical concept. Furthermore, many studies focused on a single area of the body instead of 'whole body' management and had no long-term follow-up. ${ }^{74}$ Since pain-related fear serves as a risk factor for the persistence of chronic disabling pain ${ }^{82}$, specific treatment targeting disabling psychological factors such as pain-related fear or anxiety should be conducted and may improve the long-term effectiveness. 


\section{Role of pain-related fear}

Pain-related fear is common in adolescents with CMP. ${ }^{17,18}$ In adults with CMP, the fearavoidance model (FAM) (Figure 1.3), explains the disabling role of pain-related fear. ${ }^{83}$ As a result of the growing evidence on the role of pain-related fear in youth, this framework has also been applied to paediatric chronic pain. ${ }^{84,85}$ Exposure in vivo is a cognitivebehavioural treatment that targets to restore the normal pattern of daily functioning by reducing pain-related fear and catastrophic thinking, through exposing the individual to fear-provoking daily life activities and movements. ${ }^{86}$ Several studies showed that exposure in vivo was effective in reducing disability and pain-related fear in different adult and adolescent chronic pain populations. ${ }^{86-89}$

It is now hypothesized that the FAM could also be applied to adolescents with GHSD/hEDS and exposure in vivo might be a possible treatment specifically targeting pain and disability-related problems for this group. It is hypothesized that pain-related fear and $\mathrm{GJH}$, two frequently encountered phenomena in adolescents with CMP, are important factors and can even strengthen each other in the development and maintenance of CMP leading to disabling pain and diminished daily functioning.

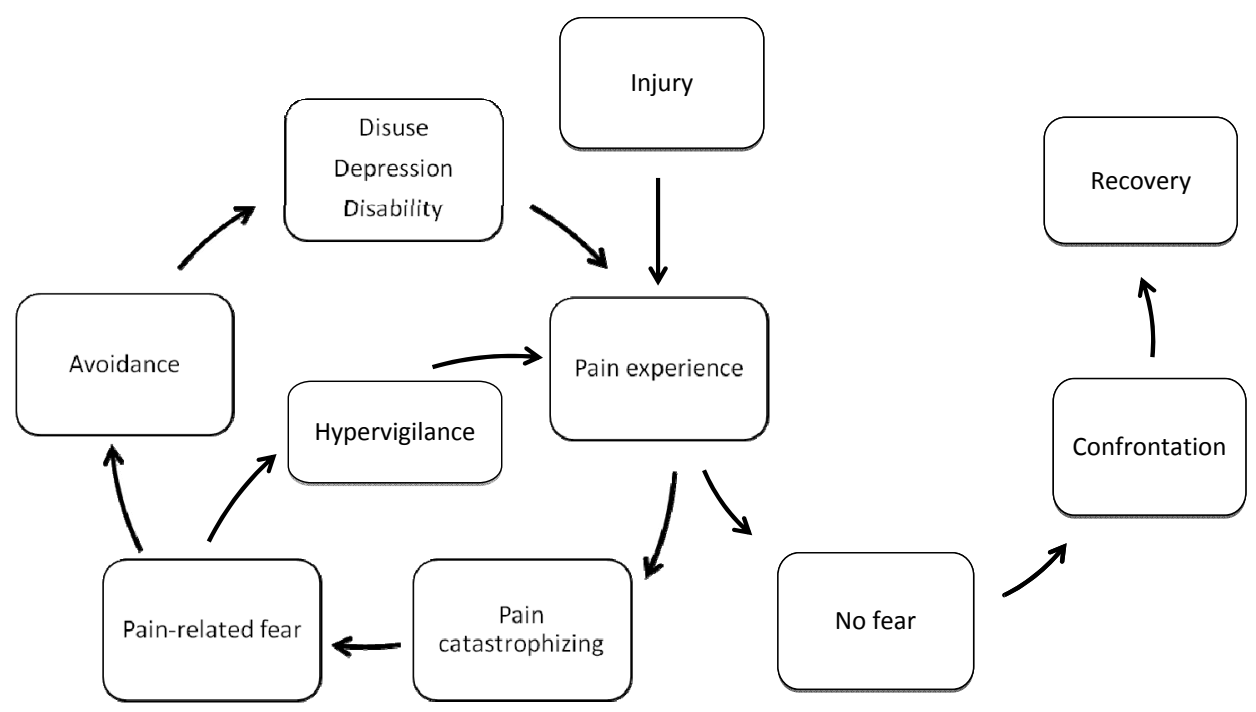

Figure 1.3 The fear-avoidance model according to Vlaeyen and Linton, $2000 .^{83}$ 


\section{Aims and outlines of this dissertation}

The objective of this dissertation is to evaluate the specific influence of both pain-related fear and GJH on physical functioning in adolescents with CMP. Knowledge of this potential negative impact of CMP and pain-related fear enables to further unravel the mechanism of disabling G-HSD/hEDS and might lead to better tailored interventions in adolescents with GHSD/hEDS.

After the introduction in chapter 1 , in chapter 2, a topical review is presented in which the current state of evidence is summarized and presented using the individual components of the fear-avoidance model as a framework. Based on that, an applied fear-avoidance model for disability in G-HSD/hEDS, is proposed as possible underlying mechanism in the relations between GJH, pain-related fear, and disability. Chapter 3 describes a cross-sectional study to evaluate whether pain-related fear has a negative influence on disability in hypermobile compared to non-hypermobile adolescents with CMP. Chapters 4 and 5 are focussing on the impact of GJH and pain-related fear on physical functioning. In chapter 4, the influence of GJH and pain-related fear on physical functioning (muscle strength, motor performance, and physical activity level [PAL]) is investigated in pain-free adolescents. Chapter 5, a case-control study, explores the influence of GJH and CMP on physical functioning (muscle strength, motor performance, and $\mathrm{PAL}$ ) in adolescents. Furthermore, the specific role of pain-related fear on physical functioning is evaluated. Chapter 6 describes an outcome study with a pre-post-test design of a multidisciplinary rehabilitation treatment consisted of a combination of physical training and exposure in vivo in hypermobile adolescents with CMP. In the final chapter 7, a general discussion reflects on the findings of the different chapters, conclusions are formulated, and recommendations for further research are proposed. 


\section{References}

1. McMahon SB, Koltzenburg M, Tracey I, Turk D. Wall and Melzack's Textbook of Pain. 6th ed: Saunders; 2013. p.

2. Woolf CJ. What is this thing called pain? J Clin Invest. 2010;120(11):3742-4.

3. Raja SN, Carr DB, Cohen M, Finnerup NB, Flor H, Gibson S, et al. The revised International Association for the Study of Pain definition of pain: concepts, challenges, and compromises. Pain. 2020;161(9):1976-82.

4. Treede RD, Rief W, Barke A, Aziz Q, Bennett MI, Benoliel R, et al. A classification of chronic pain for ICD11. Pain. 2015;156(6):1003-7.

5. Treede RD, Rief W, Barke A, Aziz Q, Bennett MI, Benoliel R, et al. Chronic pain as a symptom or a disease: the IASP Classification of Chronic Pain for the International Classification of Diseases (ICD-11). Pain. 2019;160(1):19-27.

6. Nicholas M, Vlaeyen JWS, Rief W, Barke A, Aziz Q, Benoliel R, et al. The IASP classification of chronic pain for ICD-11: chronic primary pain. Pain. 2019;160(1):28-37.

7. Breivik H, Collett B, Ventafridda V, Cohen R, Gallacher D. Survey of chronic pain in Europe: prevalence, impact on daily life, and treatment. Eur J Pain. 2006;10(4):287-333.

8. Goldberg DS, McGee SJ. Pain as a global public health priority. BMC Public Health. 2011;11:770.

9. Palermo TM, Valrie CR, Karlson CW. Family and parent influences on pediatric chronic pain: a developmental perspective. Am Psychol. 2014;69(2):142-52.

10. Finley GA, MacLaren Chorney J, Campbell L. Not small adults: the emerging role of pediatric pain services. Can J Anaesth. 2014;61(2):180-7.

11. Kaplan P. Adolescence. Boston: Houghton Miffin Company; 2004.

12. El-Metwally A, Salminen JJ, Auvinen A, Kautiainen H, Mikkelsson M. Prognosis of non-specific musculoskeletal pain in preadolescents: a prospective 4-year follow-up study till adolescence. Pain. 2004;110(3):550-9.

13. Perquin CW, Hazebroek-Kampschreur AA, Hunfeld JA, Bohnen AM, van Suijlekom-Smit LW, Passchier J, et al. Pain in children and adolescents: a common experience. Pain. 2000;87(1):51-8.

14. Kashikar-Zuck S. Treatment of children with unexplained chronic pain. Lancet. 2006;367(9508):380-2.

15. O'Sullivan P, Beales D, Jensen L, Murray K, Myers T. Characteristics of chronic non-specific musculoskeletal pain in children and adolescents attending a rheumatology outpatients clinic: a crosssectional study. Pediatr Rheumatol Online J. 2011;9(1):3.

16. Gatchel RJ, Peng YB, Peters ML, Fuchs PN, Turk DC. The biopsychosocial approach to chronic pain: scientific advances and future directions. Psychol Bull. 2007;133(4):581-624.

17. Martin AL, McGrath PA, Brown SC, Katz J. Anxiety sensitivity, fear of pain and pain-related disability in children and adolescents with chronic pain. Pain Research and Management. 2007;12(4):267-72.

18. Simons LE, Sieberg CB, Carpino E, Logan D, Berde C. The Fear of Pain Questionnaire (FOPQ): assessment of pain-related fear among children and adolescents with chronic pain. J Pain. 2011;12(6):677-86.

19. Wilson AC, Lewandowski AS, Palermo TM. Fear-avoidance beliefs and parental responses to pain in adolescents with chronic pain. Pain Res Manag. 2011;16(3):178-82.

20. Sohrbeck-Nohr O, Kristensen JH, Boyle E, Remvig L, Juul-Kristensen B. Generalized joint hypermobility in childhood is a possible risk for the development of joint pain in adolescence: a cohort study. BMC Pediatr. 2014;14:302.

21. Tobias JH, Deere K, Palmer S, Clark EM, Clinch J. Joint hypermobility is a risk factor for musculoskeletal pain during adolescence: findings of a prospective cohort study. Arthritis Rheum. 2013;65(4):1107-15.

22. Siegel DM, Janeway D, Baum J. Fibromyalgia syndrome in children and adolescents: clinical features at presentation and status at follow-up. Pediatrics. 1998;101(3 Pt 1):377-82.

23. Ting TV, Hashkes PJ, Schikler K, Desai AM, Spalding S, Kashikar-Zuck S. The role of benign joint hypermobility in the pain experience in Juvenile Fibromyalgia: an observational study. Pediatr Rheumatol Online J. 2012;10(1):16.

24. Wang TJ. Concept analysis of functional status. Int J Nurs Stud. 2004;41(4):457-62.

25. King S, Chambers CT, Huguet A, MacNevin RC, McGrath PJ, Parker L, et al. The epidemiology of chronic pain in children and adolescents revisited: a systematic review. Pain. 2011;152(12):2729-38. 
26. Mikkelsson M, El-Metwally A, Kautiainen H, Auvinen A, Macfarlane GJ, Salminen JJ. Onset, prognosis and risk factors for widespread pain in schoolchildren: a prospective 4-year follow-up study. Pain. 2008;138(3):681-7.

27. Brattberg G. Do pain problems in young school children persist into early adulthood? A 13-year followup. Eur J Pain. 2004;8(3):187-99.

28. Konijnenberg AY, Uiterwaal CS, Kimpen JL, van der Hoeven J, Buitelaar JK, de Graeff-Meeder ER. Children with unexplained chronic pain: substantial impairment in everyday life. Arch Dis Child. 2005;90(7):680-6.

29. Huguet A, Miro J. The severity of chronic pediatric pain: an epidemiological study. J Pain. 2008;9(3): 226-36.

30. Hunfeld JA, Perquin CW, Duivenvoorden HJ, Hazebroek-Kampschreur AA, Passchier J, van Suijlekom-Smit LW, et al. Chronic pain and its impact on quality of life in adolescents and their families. J Pediatr Psychol. 2001;26(3):145-53.

31. Kashikar-Zuck S, Goldschneider KR, Powers SW, Vaught MH, Hershey AD. Depression and functional disability in chronic pediatric pain. Clin J Pain. 2001;17(4):341-9.

32. Perquin CW, Hazebroek-Kampschreur AA, Hunfeld JA, van Suijlekom-Smit LW, Passchier J, van der Wouden JC. Chronic pain among children and adolescents: physician consultation and medication use. Clin J Pain. 2000;16(3):229-35.

33. Masiero S, Carraro E, Sarto D, Bonaldo L, Ferraro C. Healthcare service use in adolescents with nonspecific musculoskeletal pain. Acta Paediatr. 2010;99(8):1224-8.

34. Sleed M, Eccleston C, Beecham J, Knapp M, Jordan A. The economic impact of chronic pain in adolescence: methodological considerations and a preliminary costs-of-illness study. Pain. 2005;119(13):183-90.

35. Groenewald CB, Essner BS, Wright D, Fesinmeyer MD, Palermo TM. The economic costs of chronic pain among a cohort of treatment-seeking adolescents in the United States. J Pain. 2014;15(9):925-33.

36. Beighton P, Grahame R, Bird H. Musculoskeletal Features of Hypermobility and Their Management. . Hypermobility of Joints. 4th ed. London: Springer; 2012. p. 65-99.

37. Murray KJ. Hypermobility disorders in children and adolescents. Best Practice \& Research Clinical Rheumatology. 2006;20(2):329-51.

38. Grahame R. Joint hypermobility syndrome pain. Curr Pain Headache Rep. 2009;13(6):427-33.

39. Adib N, Davies K, Grahame R, Woo P, Murray KJ. Joint hypermobility syndrome in childhood. A not so benign multisystem disorder? Rheumatology (Oxford). 2005;44(6):744-50.

40. Meyer KJ, Chan C, Hopper L, Nicholson LL. Identifying lower limb specific and generalised joint hypermobility in adults: validation of the Lower Limb Assessment Score. BMC Musculoskelet Disord. 2017;18(1):514.

41. Nicholson LL, Chan C. The Upper Limb Hypermobility Assessment Tool: A novel validated measure of adult joint mobility. Musculoskelet Sci Pract. 2018;35:38-45.

42. Juul-Kristensen B, Schmedling K, Rombaut L, Lund H, Engelbert RH. Measurement properties of clinical assessment methods for classifying generalized joint hypermobility-A systematic review. Am J Med Genet C Semin Med Genet. 2017;175(1):116-47.

43. Folci M, Capsoni F. Arthralgias, fatigue, paresthesias and visceral pain: can joint hypermobility solve the puzzle? A case report. BMC Musculoskelet Disord. 2016;17:58.

44. Scheper MC, de Vries JE, Juul-Kristensen B, Nollet F, Engelbert RH. The functional consequences of generalized joint hypermobility: a cross-sectional study. BMC Musculoskeletal Disorders. 2014;15:243.

45. Scheper M, de Vries J, Beelen A, de Vos R, Nollet F, Engelbert R. Generalized joint hypermobility, muscle strength and physical function in healthy adolescents and young adults. Curr Rheumatol Rev. 2014; 10(2):117-25.

46. Jindal P, Narayan A, Ganesan S, MacDermid JC. Muscle strength differences in healthy young adults with and without generalized joint hypermobility: a cross-sectional study. BMC Sports Sci Med Rehabil. 2016; 8:12.

47. Jensen BR, Olesen AT, Pedersen MT, Kristensen JH, Remvig L, Simonsen EB, et al. Effect of generalized joint hypermobility on knee function and muscle activation in children and adults. Muscle Nerve. 2013;48(5):762-9. 
48. Clinch J, Deere K, Sayers A, Palmer S, Riddoch C, Tobias JH, et al. Epidemiology of generalized joint laxity (hypermobility) in fourteen-year-old children from the UK: a population-based evaluation. Arthritis Rheum. 2011;63(9):2819-27.

49. Scheper MC, de Vries JE, de Vos R, Verbunt J, Nollet F, Engelbert RH. Generalized joint hypermobility in professional dancers: a sign of talent or vulnerability? Rheumatology (Oxford). 2013;52(4):651-8.

50. Larsson LG, Baum J, Mudholkar GS, Kollia GD. Benefits and disadvantages of joint hypermobility among musicians. N Engl J Med. 1993;329(15):1079-82.

51. Castori M, Tinkle B, Levy H, Grahame R, Malfait F, Hakim A. A framework for the classification of joint hypermobility and related conditions. Am J Med Genet C Semin Med Genet. 2017;175(1):148-57.

52. Grahame R, Bird HA, Child A. The revised (Brighton 1998) criteria for the diagnosis of benign joint hypermobility syndrome (BJHS). J Rheumatol. 2000;27(7):1777-9.

53. Malfait F, Hakim AJ, De Paepe A, Grahame R. The genetic basis of the joint hypermobility syndromes. Rheumatology (Oxford). 2006;45(5):502-7.

54. Malfait F, Francomano C, Byers P, Belmont J, Berglund B, Black J, et al. The 2017 international classification of the Ehlers-Danlos syndromes. Am J Med Genet C Semin Med Genet. 2017;175(1):8-26.

55. Beighton P, De Paepe A, Steinmann B, Tsipouras P, Wenstrup RJ. Ehlers-Danlos syndromes: revised nosology, Villefranche, 1997. Ehlers-Danlos National Foundation (USA) and Ehlers-Danlos Support Group (UK). Am J Med Genet. 1998;77(1):31-7.

56. Scheper MC. Hypermobility Syndrome and Ehlers-Danlos Syndrome (Hypermobility type): Unraveling the concepts of disability during childhood: University of Amsterdam; 2019.

57. Fatoye F, Palmer S, Macmillan F, Rowe P, van der Linden M. Proprioception and muscle torque deficits in children with hypermobility syndrome. Rheumatology (Oxford). 2009;48(2):152-7.

58. Hanewinkel-van Kleef YB, Helders PJ, Takken T, Engelbert RH. Motor performance in children with generalized hypermobility: the influence of muscle strength and exercise capacity. Pediatr Phys Ther. 2009;21(2):194-200.

59. Engelbert RH, van Bergen M, Henneken T, Helders PJ, Takken T. Exercise tolerance in children and adolescents with musculoskeletal pain in joint hypermobility and joint hypomobility syndrome. Pediatrics. 2006;118(3):690-6.

60. Schubert-Hjalmarsson E, Ohman A, Kyllerman M, Beckung E. Pain, balance, activity, and participation in children with hypermobility syndrome. Pediatr Phys Ther. 2012;24(4):339-44.

61. Scheper MC, de Vries JE, Verbunt J, Engelbert RH. Chronic pain in hypermobility syndrome and EhlersDanlos syndrome (hypermobility type): it is a challenge. J Pain Res. 2015;8:591-601.

62. Engelbert RH, Bank RA, Sakkers RJ, Helders PJ, Beemer FA, Uiterwaal CS. Pediatric generalized joint hypermobility with and without musculoskeletal complaints: a localized or systemic disorder? Pediatrics. 2003;111(3):248-54.

63. Pacey V, Tofts L, Adams RD, Munns CF, Nicholson LL. Quality of life prediction in children with joint hypermobility syndrome. J Paediatr Child Health. 2015;51(7):689-95.

64. Tinkle BT. Symptomatic joint hypermobility. Best Pract Res Clin Rheumatol. 2020:101508.

65. Voermans NC, Knoop H. Both pain and fatigue are important possible determinants of disability in patients with the Ehlers-Danlos syndrome hypermobility type. Disabil Rehabil. 2011;33(8):706-7.

66. Di Stefano G, Celletti C, Baron R, Castori M, Di Franco M, La Cesa S, et al. Central sensitization as the mechanism underlying pain in joint hypermobility syndrome/Ehlers-Danlos syndrome, hypermobility type. Eur J Pain. 2016;20(8):1319-25.

67. Scheper MC, Pacey V, Rombaut L, Adams RD, Tofts L, Calders P, et al. Generalized Hyperalgesia in Children and Adults Diagnosed With Hypermobility Syndrome and Ehlers-Danlos Syndrome Hypermobility Type: A Discriminative Analysis. Arthritis Care Res (Hoboken). 2017;69(3):421-9.

68. Baeza-Velasco C, Bourdon C, Montalescot L, de Cazotte C, Pailhez G, Bulbena A, et al. Low- and highanxious hypermobile Ehlers-Danlos syndrome patients: comparison of psychosocial and health variables. Rheumatol Int. 2018;38(5):871-8.

69. Celletti C, Castori M, La Torre G, Camerota F. Evaluation of kinesiophobia and its correlations with pain and fatigue in joint hypermobility syndrome/Ehlers-Danlos syndrome hypermobility type. Biomed Res Int. 2013;2013:580460. 
70. Bulbena A, Gago J, Pailhez G, Sperry L, Fullana MA, Vilarroya O. Joint hypermobility syndrome is a risk factor trait for anxiety disorders: a 15-year follow-up cohort study. Gen Hosp Psychiatry. 2011;33(4): 363-70.

71. Javadi Parvaneh V, Modaress S, Zahed G, Rahmani K, Shiari R. Prevalence of generalized joint hypermobility in children with anxiety disorders. BMC Musculoskelet Disord. 2020;21(1):337.

72. Smith TO, Easton V, Bacon H, Jerman E, Armon K, Poland F, et al. The relationship between benign joint hypermobility syndrome and psychological distress: a systematic review and meta-analysis. Rheumatology (Oxford). 2014;53(1):114-22.

73. Engelbert RH, Juul-Kristensen B, Pacey V, de Wandele I, Smeenk S, Woinarosky N, et al. The evidencebased rationale for physical therapy treatment of children, adolescents, and adults diagnosed with joint hypermobility syndrome/hypermobile Ehlers Danlos syndrome. Am J Med Genet C Semin Med Genet. 2017;175(1):158-67.

74. Palmer S, Davey I, Oliver L, Preece A, Sowerby L, House S. The effectiveness of conservative interventions for the management of syndromic hypermobility: a systematic literature review. Clin Rheumatol. 2020.

75. Sahin N, Baskent A, Cakmak A, Salli A, Ugurlu H, Berker E. Evaluation of knee proprioception and effects of proprioception exercise in patients with benign joint hypermobility syndrome. Rheumatol Int. 2008;28(10):995-1000.

76. Ferrell WR, Tennant N, Sturrock RD, Ashton L, Creed G, Brydson G, et al. Amelioration of symptoms by enhancement of proprioception in patients with joint hypermobility syndrome. Arthritis Rheum. 2004;50(10):3323-8.

77. Barton LM, Bird HA. Improving pain by the stabilization of hyperlax joints. J Orthop Rheumatol. 1996;9:46-51.

78. Pacey V, Tofts L, Adams RD, Munns CF, Nicholson LL. Exercise in children with joint hypermobility syndrome and knee pain: a randomised controlled trial comparing exercise into hypermobile versus neutral knee extension. Pediatr Rheumatol Online J. 2013;11(1):30.

79. Bathen $T$, Hangmann AB, Hoff M, Andersen LO, Rand-Hendriksen S. Multidisciplinary treatment of disability in ehlers-danlos syndrome hypermobility type/hypermobility syndrome: A pilot study using a combination of physical and cognitive-behavioral therapy on 12 women. Am J Med Genet A. 2013;161(12):3005-11.

80. Rahman A, Daniel C, Grahame R. Efficacy of an out-patient pain management programme for people with joint hypermobility syndrome. Clin Rheumatol. 2014;33(11):1665-9.

81. Bale $P$, Easton V, Bacon H, Jerman E, Watts L, Barton $G$, et al. The effectiveness of a multidisciplinary intervention strategy for the treatment of symptomatic joint hypermobility in childhood: a randomised, single Centre parallel group trial (The Bendy Study). Pediatr Rheumatol Online J. 2019;17(1):2.

82. Turk DC, Wilson HD. Fear of pain as a prognostic factor in chronic pain: conceptual models, assessment, and treatment implications. Curr Pain Headache Rep. 2010;14(2):88-95.

83. Vlaeyen JW, Linton SJ. Fear-avoidance and its consequences in chronic musculoskeletal pain: a state of the art. Pain. 2000;85(3):317-32.

84. Simons LE, Kaczynski KJ. The Fear Avoidance model of chronic pain: examination for pediatric application. J Pain. 2012;13(9):827-35.

85. Asmundson GJ, Noel M, Petter M, Parkerson HA. Pediatric fear-avoidance model of chronic pain: foundation, application and future directions. Pain Res Manag. 2012;17(6):397-405.

86. den Hollander M, Goossens M, de Jong J, Ruijgrok J, Oosterhof J, Onghena P, et al. Expose or protect? A randomized controlled trial of exposure in vivo vs pain-contingent treatment as usual in patients with complex regional pain syndrome type 1. Pain. 2016;157(10):2318-29.

87. Leeuw M, Goossens ME, van Breukelen GJ, de Jong JR, Heuts PH, Smeets RJ, et al. Exposure in vivo versus operant graded activity in chronic low back pain patients: results of a randomized controlled trial. Pain. 2008;138(1):192-207.

88. van den Bogert - Dekker C. Adolescent Chronic Pain. Rehabilitation treatment targeting pain-related fear [dissertation]. Heerlen (NL): Maastricht University; 2018.

89. de Jong JR, Vangronsveld K, Peters ML, Goossens ME, Onghena P, Bulte I, et al. Reduction of pain-related fear and disability in post-traumatic neck pain: a replicated single-case experimental study of exposure in vivo. J Pain. 2008;9(12):1123-34. 



\section{Chapter 2}

Exploring the underlying mechanism of pain-related disability in hypermobile adolescents with chronic

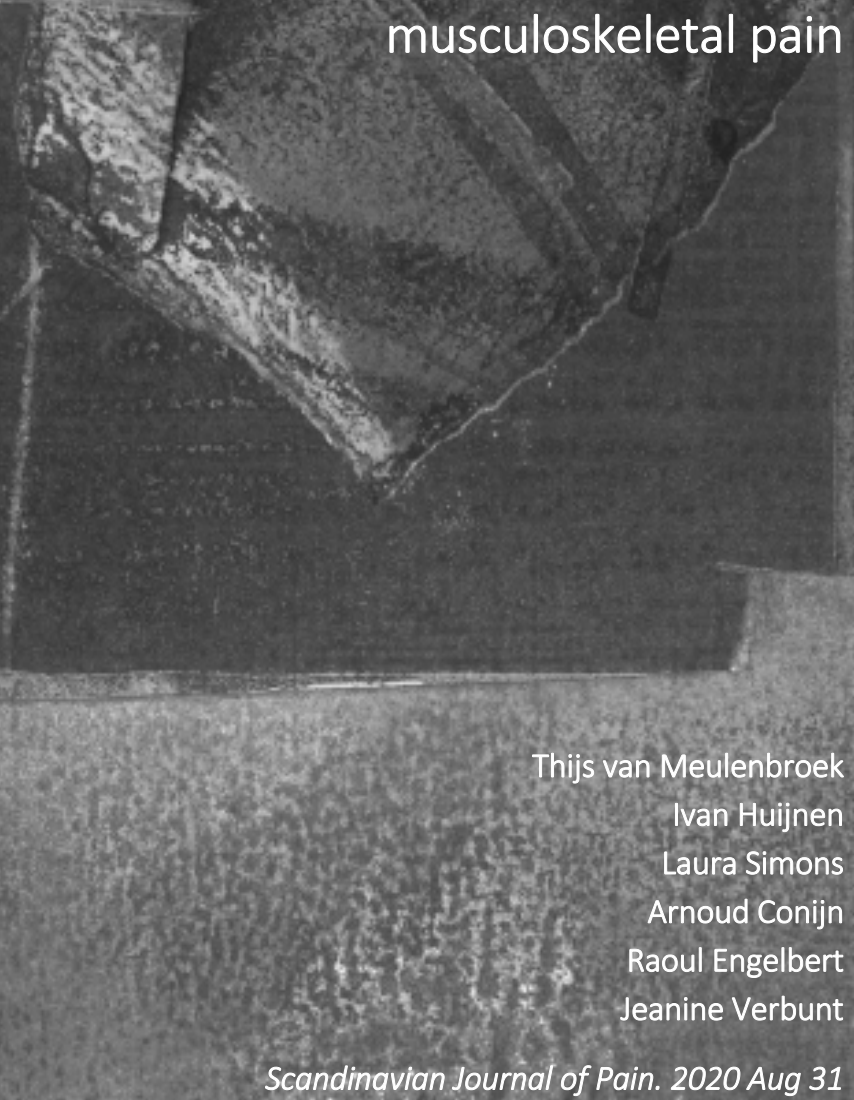




\section{Abstract}

\section{Objectives}

A significant proportion of adolescents with chronic musculoskeletal pain (CMP) experience difficulties in physical functioning, mood, and social functioning, contributing to diminished quality of life. Generalized joint hypermobility (GJH) is a risk factor for developing CMP with a striking 35-48\% of patients with CMP reporting GJH. In case GJH occurs with one or more musculoskeletal manifestations such as chronic pain, trauma, disturbed proprioception, and joint instability, it is referred to as generalized hypermobility spectrum disorder (G-HSD). Similar characteristics have been reported in children and adolescents with the hypermobile Ehlers-Danlos syndrome (hEDS). In the management of CMP, a biopsychosocial approach is recommended as several studies have confirmed the impact of psychosocial factors in the development and maintenance of CMP. The fear-avoidance model (FAM) is a cognitive-behavioural framework that describes the role of pain-related fear as a determinant of CMP-related disability.

\section{Content}

Pubmed was used to identify existing relevant literature focusing on chronic musculoskeletal pain, generalized joint hypermobility, pain-related fear, and disability. Relevant articles were cross-referenced to identify articles possibly missed during the primary screening. In this paper the current state of scientific evidence is presented for each individual component of the FAM in hypermobile adolescents with and without CMP. Based on this overview, the FAM is proposed explaining a possible underlying mechanism in the relations between GJH, pain-related fear, and disability.

\section{Summary and outlook}

It is assumed that GJH seems to make you more vulnerable for injury and experiencing more frequent musculoskeletal pain. But in addition, a vulnerability for heightened painrelated fear is proposed as an underlying mechanism explaining the relationship between GJH and disability. Further scientific confirmation of this applied FAM is warranted to further unravel the underlying mechanism.

In explaining disability in individuals with G-HSD/hEDS, it is important to focus on both the physical components related to joint hypermobility, in tandem with the psychological components such as pain-related fear, catastrophizing thoughts, and generalized anxiety. 


\section{Introduction}

In the Netherlands, $25 \%$ of schoolchildren and adolescents have chronic pain. ${ }^{1}$ Internationally, prevalence rates for chronic musculoskeletal pain (CMP) in schoolchildren and adolescents vary between 4 and $40 \%{ }^{2}$ CMP refers to pain lasting longer than 3 months and it is localized in muscular, ligamentous, and bony structures. ${ }^{3}$ In approximately $40 \%$ of adolescents with CMP, pain has a disabling impact on physical functioning, mood, and social functioning, with concomitant reductions in quality of life. . $^{3,4}$

Generalized joint hypermobility $(\mathrm{GJH})$ is a non-symptomatic condition characterized by increased range of motion in multiple joints. The prevalence of GJH in the general Dutch child population (10-16 years) is $25 \%$ (cut-off Beighton score $\geq 4$ ) , whereas $35 \%$ of Dutch adolescents (12-21 years) with disabling CMP have GJH (cut-off Beighton score $\geq 5$ ). ${ }^{6} \mathrm{GJH}$ associated with soft tissue injury, joint instability, and CMP was referred to as joint hypermobility syndrome (JHS). ${ }^{7}$ However, a lack of clarity existed on the exact labelling of hypermobility and pain, as JHS showed overlap with the hypermobility type of EhlersDanlos syndrome (EDS-HT). ${ }^{8}$ Therefore, a recently consensus statement has been published with a unified set of criteria. JHS is now referred to as generalized hypermobility spectrum disorder (G-HSD) ${ }^{9}$ and EDS-HT is now referred to as hypermobile EDS (hEDS) ${ }^{10}$ (Figure 2.1). Both conditions have no specific genetic profile and in the present article it is referred to as G-HSD/hEDS. Although the increased prevalence of GJH in disabled adolescents with CMP is striking, the underlying mechanism explaining the relation between G-HSD/hEDS and pain-related disability is currently still unclear.

In adults, pain-related fear plays a disabling role in the development and maintenance of CMP. ${ }^{11}$ The fear-avoidance model (FAM) is a biopsychosocial model (Figure 2.2), which explains this disabling role of pain-related fear. ${ }^{12}$ It states that pain experience can be interpreted in two different ways, often in response to an injury; although, it can also begin spontaneously. First, pain can be experienced as non-threatening and pain is considered as part of a life experience. As a consequence, a person will confront and accept pain and perform activities as usual thus maintaining his/her habitual activity pattern. Secondly, pain can be interpreted as threatening based on catastrophic thoughts causing pain-related fear, which in turn leads to avoidance behaviour and hypervigilance. Long-term avoidance may lead to disuse, depression, and disability, and these long-term consequences may decrease the pain threshold at which ensuing pain will be experienced ${ }^{11}$, thus causing a vicious circle. ${ }^{12}$ Recently, this framework has been applied to paediatric chronic pain ${ }^{13,14}$ and extended to incorporate parents fear and behaviours. ${ }^{15}$ Pain-related fear is also present in individuals with G-HSD/hEDS. ${ }^{16,17}$ It can be hypothesized that GJH and pain-related fear, two frequently encountered 
phenomena in adolescents with CMP, are important factors in the development and maintenance of CMP leading to disabling pain.

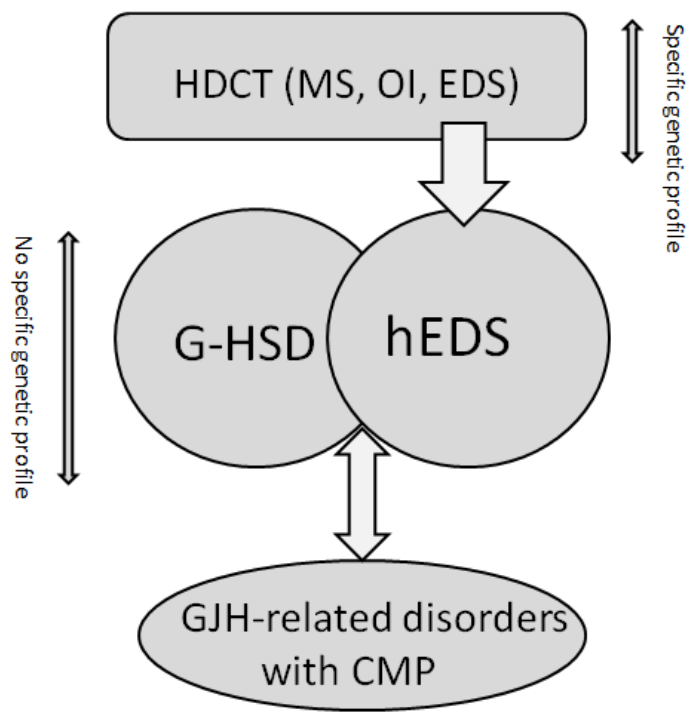

Figure 2.1 Different types of symptomatic GJH-related disorders.

Abbreviations: $\mathrm{HDCT}$ = hereditary diseases of the connective tissue; $\mathrm{MS}=$ Marfan syndrome; $\mathrm{OI}=$ osteogenesis imperfecta; EDS = Ehlers-Danlos syndrome; G-HSD = generalized hypermobility spectrum disorder; hEDS = hypermobile Ehlers-Danlos syndrome; GJH-related = generalized joint hypermobility-related; $\mathrm{CMP}=$ chronic musculoskeletal pain.

\section{Aim}

The aim of this topical review is to integrate knowledge from different research fields of expertise in CMP, hypermobility-related disorders, and rehabilitation medicine. The current state of evidence on physical and psychological functioning in G-HSD/hEDS is summarized and presented using the individual components of the FAM as a framework. Based on this, a vulnerability for heightened pain-related fear and generalized anxiety among individuals with G-HSD/hEDS as a potential underlying mechanism explaining the relation between GJH and disability is discussed and a FAM applied to G-HSD/hEDS in adolescents is proposed. 


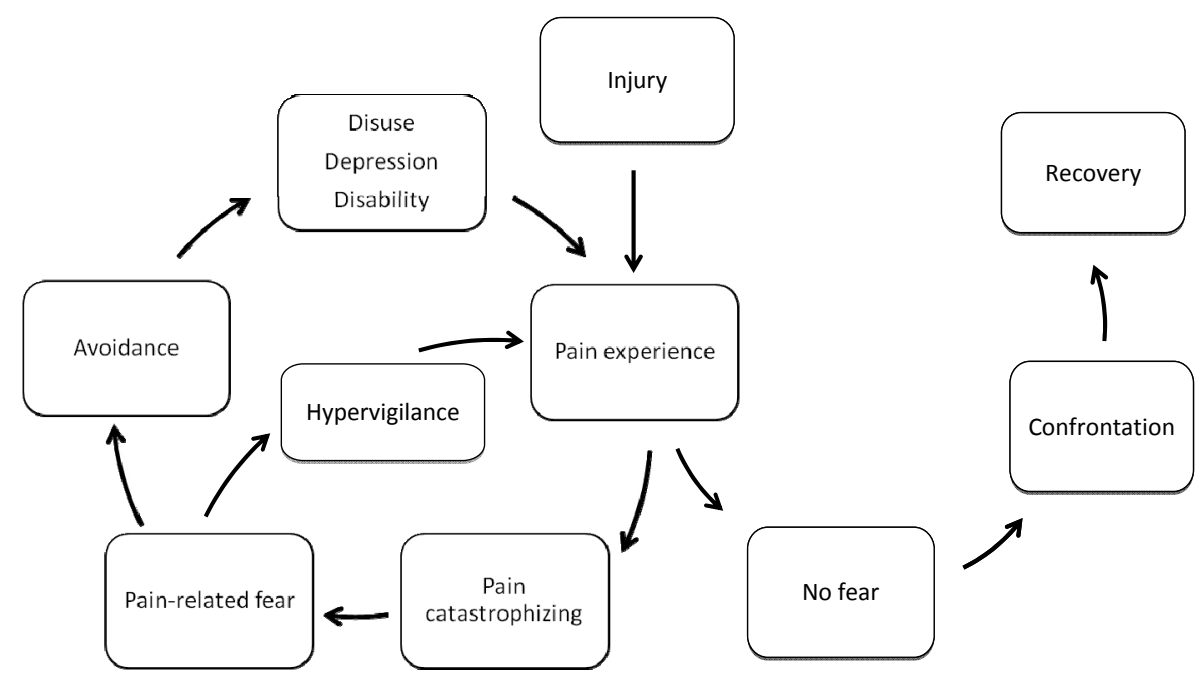

Figure 2.2 The fear-avoidance model according to Vlaeyen and Linton, 2000. ${ }^{12}$

\section{Methods}

Pubmed (https://www.ncib.nlm.nih.gov/pubmed/) was used to identify existing literature on the different topics "generalized joint hypermobility, hypermobile EhlersDanlos syndrome, generalized hypermobility spectrum disorder, joint hypermobility syndrome, and chronic musculoskeletal pain, fatigue, proprioception, balance, muscle strength, disability, psychological functioning, depression, anxiety, pain-related fear, activity limitations, physical activity". Relevant publications (original articles, case reports, editorial letters, reviews, and meta-analyses) published in English were included if existing evidence were related to the individuals components of the FAM. In addition, references of the included articles were cross-referenced to identify more articles of interest. There were no restraints for the year of publication.

\section{Results}

\section{Evidence for the fear-avoidance model components related to G-HSD/hEDS}

In the next paragraphs, the current scientific evidence for the different components of the FAM (Figure 2.2) to explain functioning among adolescents with G-HSD/hEDS will be discussed. 


\section{Injury}

Individuals with G-HSD/hEDS have higher incidence of injuries, such as sprain, tendon injuries or dislocation, compared to non-hypermobile pain-free peers. ${ }^{18-20}$ In addition, micro-traumas (due to joint instability or connective tissue fragility) might occur as a result of repetitive injuries on joint surfaces, which may lead to adaptations and compensation of movement patterns, resulting in overload in other areas of the musculoskeletal system. ${ }^{21}$ Recovery from injury seems more prolonged in individuals with G-HSD/hEDS compared to non-hypermobile pain-free peers. In a 5-year follow up study in ballet dancers G-HSD/hEDS was associated with a significant greater risk of a prolonged recovery period after injury compared to ballet dancers without GHSD/hEDS. ${ }^{20}$ Furthermore, individuals with asymptomatic GJH have an increased risk of knee injury during contact sports, such as soccer or basketball. ${ }^{22}$ In conclusion, individuals with asymptomatic GJH and G-HSD/hEDS as compared to others have an increased risk of injury. Furthermore, individuals with G-HSD/hEDS have delayed recovery after injury compared to non-hypermobile pain-free peers.

\section{Pain experience}

In G-HSD/hEDS joint pain is one of the most common complaints ${ }^{23,24}$ and recurrent musculoskeletal pain is an essential part of the classification of G-HSD/hEDS., ${ }^{9,10}$ The most commonly reported pain areas are the neck, shoulders, knees, and ankles. ${ }^{25,26}$ Pain severity is one of two important potential determinants of disability. ${ }^{27}$ Studies also showed that exercise-induced muscle pain is a distinctive symptom in individuals with $\mathrm{G}$ HSD/hEDS, mostly in the knee and the shoulder. ${ }^{25,28}$ Recently, the presence of generalized hyperalgesia has been documented in individuals with G-HSD/hEDS compared to healthy controls. ${ }^{29,30}$ This could suggest that pain experience is a result of abnormal central processing of the nociceptive input, possibly associated with descending modulatory system dysfunction and peripheral involvement such as a reduction in the pain threshold. As a result, pain could occur even in response to nonpainful stimuli. ${ }^{31}$ Two longitudinal cohort studies showed that during adolescence, GJH represents a risk factor for the development of musculoskeletal pain. Tobias et al. ${ }^{32}$ found that GJH had a 2-fold increased risk factor of musculoskeletal pain in shoulder, knee or ankle/foot. In addition, Sohrbeck-Nohr et al. ${ }^{33}$ found that GJH had a 3 times higher risk of developing joint pain in adolescence. These findings are consistent with those in previous studies showing that GJH was a predictor of pain recurrence and pain persistence in adolescence. $^{34,35}$

In summary, individuals with G-HSD/hEDS experience more frequent pain and generalized hyperalgesia might contribute to the heightened occurrence of pain. 
Furthermore, GJH represents a risk factor for the development of persistent musculoskeletal pain.

\section{Pain catastrophizing}

Pain catastrophizing is defined as an exaggerated negative interpretation of actual and anticipated pain experience. ${ }^{36,37}$ For example, individuals with high levels of catastrophizing are convinced that pain is threatening, which means that their body is vulnerable and needs careful protection to prevent further harm. In a recent crosssectional study in adults with hEDS half of patients reported high levels of pain catastrophizing (Pain Catastrophizing Scale [PCS] >30). ${ }^{16}$ In addition, a study of Rahman et al. $^{38}$ performed in adults with G-HSD showed high levels of pain catastrophizing (average PCS score of 27.9; scale 0-42). Thus, maladaptive cognitions about pain are present in adults with G-HSD/hEDS. No study in adolescents with G-HSD/hEDS are present in the literature yet.

\section{Pain-related fear}

Pain-related fear is defined as fear that emerges when stimuli that are related to pain are perceived as a main threat, such as fear of movement, fear of (re)injury or fear of pain. ${ }^{11}$ In a general population of individuals with G-HSD/hEDS aged 15-61 years, painrelated fear is a common characteristic. ${ }^{16,17}$ In previous cross-sectional studies an overwhelming majority of individuals with G-HSD/hEDS met the cut-off point for heightened fear of movement $\left(75 \%{ }^{16}\right.$ and $93 \%{ }^{17}$; Tampa Scale for Kinesiophobia [TSK] score $>37$ ). It has also been shown that fear of injury is a barrier to exercise in patients with G-HSD/hEDS. ${ }^{39}$ This finding was confirmed by the results of a qualitative semistructured interview study ${ }^{40}$, in which women with G-HSD reported that fear of pain or (re)injury reduced or altered particular activities. In a recent cross-sectional study $61 \%$ of adolescents with CMP had clinically elevated levels of pain-related fear, assessed with the Fear of Pain Questionnaire-Child report (FOPQ-C >50). ${ }^{6}$ However, there were no significant differences on levels of pain-related fear between hypermobile and nonhypermobile adolescents with CMP. ${ }^{6}$ Recent published data in adolescents with asymptomatic GJH showed that the negative impact of pain-related fear, measured as perceived harmfulness, was not more pronounced in asymptomatic GJH compared to non-hypermobile pain-free peers. ${ }^{41}$ In summary, pain-related fear is a common symptom in G-HSD/hEDS, but was not higher in adolescents with asymptomatic GJH compared to non-hypermobile pain-free peers. 


\section{Hypervigilance}

Hypervigilance is increased attention toward the source of the threat, such as pain or somatic sensations. ${ }^{11}$ To the best of our knowledge, no studies have investigated the relationship between hypervigilance and G-HSD/hEDS in adolescents and adults. The pain vigilance and awareness questionnaire (PVAC) was developed as a broad measure of attention to pain and could be used in further research in adolescents with G-HSD/hEDS. ${ }^{37}$

\section{Avoidance behaviour}

Avoidance behaviour refers to behaviour aimed at postponing or preventing a potentially aversive situation from occurring ${ }^{42}$ and might be influenced by the beliefs and recommendations of healthcare professionals. ${ }^{43}$ In some situations avoidance behaviour could be a strategy for a specific medical condition influencing daily life functioning, such as acute musculoskeletal pain. However, in children and adolescents with G-HSD/hEDS avoidance behaviour is maladaptive. For instance, children with G-HSD/hEDS report a tendency to avoid dynamic activities, such as sports or outdoor games requiring more joint stability. In addition, children with G-HSD/hEDS also reported a higher need to rest, which may reduce the possibility of being active in daily life activities and might be a result of avoidance behaviour. ${ }^{44}$ Furthermore, as previously mentioned, activity-triggered pain is often seen in children and adolescents with G-HSD/hEDS. ${ }^{25}$ In addition, pain (87\%), fatigue (78\%), and fear of injury (50\%) are common reported barriers to exercise in individuals with G-HSD/hEDS. ${ }^{39}$ Adolescents and young adults with asymptomatic GJH tend to avoid dynamic activities (for instance sports) and prefer static activities (such as cycling and walking during leisure time), which require less balance and stress on ligamentous joints. ${ }^{45}$ In summary, avoidance behaviour is reported in both children and adolescents with asymptomatic GJH and with G-HSD/hEDS.

\section{Disuse}

The term disuse can be described as performing at a reduced level of physical activity (PA) in daily life and according to the FAM is a result of avoiding movements and activities (avoidance behaviour). ${ }^{46}$ In individuals with G-HSD/hEDS several cross-sectional studies demonstrate reduced levels of PA when compared to pain-free controls. ${ }^{25,44,47}$ Children and adolescents with G-HSD/hEDS report more withdrawal from physical education, sports or outdoor games, and physical related activities in their social time. ${ }^{25,44}$ Furthermore, children and adolescents with G-HSD/hEDS report playing more non-sports (such as board games or electronic games) and report needing more time to 
rest. ${ }^{44}$ In cross-sectional studies, no differences in total PA levels appeared to be present between asymptomatic GJH and non-hypermobile pain-free controls (as assessed based on the Short QUestionnaire to ASess Health-enhancing physical activity ${ }^{45}$ nor accelerometry. ${ }^{41}$ However, adolescents with GJH tend to prefer walking and cycling over sports and outdoor activities when compared to non-hypermobile pain-free peers. ${ }^{45}$

A long-term consequence of physical inactivity is physical deconditioning ${ }^{46}$, which can be expressed in decreased muscle strength and reduced cardiorespiratory fitness. Individuals with G-HSD/hEDS showed a decrease in lower and upper extremity muscle strength as compared to non-hypermobile pain-free peers. ${ }^{47-49}$ It is important to note that these results might be influenced by pain experienced during testing and psychological factors, such as pain catastrophizing and pain-related fear. Individuals might decide to stop or perform submaximally, resulting in a lower score. ${ }^{50}$ Decreased muscle strength is also found to be associated with more activity limitations in adults with G-HSD/hEDS. ${ }^{49}$ In addition, reduced cardiorespiratory fitness has been observed in children and adolescents with G-HSD/hEDS compared to non-hypermobile pain-free peers. ${ }^{51,52}$ It remains unclear if decreased muscle strength and reduced cardiorespiratory fitness in individuals with G-HSD/hEDS may be long-term consequences of avoidance behaviour due to CMP or an inherent characteristic of having a symptomatic GJH-related disorder. Some studies performed in children and adolescents with asymptomatic GJH indeed showed decreased muscle strength ${ }^{45,53}$ and lower levels of cardiorespiratory fitness ${ }^{54}$ compared to non-hypermobile pain-free peers, while other studies could not confirm this. ${ }^{41,55,56}$

In summary, disuse is shown in individuals with G-HSD/hEDS, which might be a result of avoidance behaviour. Disuse will eventually result in physical deconditioning characterized by decreased muscle strength and reduced cardiorespiratory fitness which significantly influence each other. It can be hypothesized that decreased muscle strength and reduced cardiorespiratory fitness in individuals with G-HSD/hEDS are a result of deconditioning after pain has started or submaximal performance due to pain interference during testing and psychological factors, such as pain catastrophizing and pain-related fear.

\section{Depression}

Depression refers to a condition that negatively affects mood, self-concept, and selfesteem $^{57}$ and is more common in adults with G-HSD/hEDS compared to nonhypermobile pain-free peers. ${ }^{58,59}$ However, a cross-sectional study in young adults with and without asymptomatic GJH using multiple linear regression models showed no significant association between $\mathrm{GJH}$ and depression as measured with the HADS depression subscale. ${ }^{54}$ 
In conclusion, depression among adults with G-HSD/hEDS is common compared to nonhypermobile pain-free individuals. However, there is a notable lack of evidence in childhood populations with G-HSD/hEDS regarding depression. ${ }^{58}$

\section{Disability and quality of life}

Disability is an umbrella term that encompasses not only physical impairments, but also activity and participation limitations across home and school. ${ }^{11,60}$ Within the FAM, disability is conceptualized as a long-term consequence of prolonged avoidance behaviour. In studies of adolescents and adults with G-HSD/hEDS both pain and fatigue are associated with disability. ${ }^{27,60}$ Furthermore, a study reported that children and adolescents with G-HSD/hEDS have general difficulties at school and specifically handwriting problems. ${ }^{25}$ When compared to non-hypermobile adolescents with CMP, no differences in the level of disability emerged. ${ }^{6}$ A possible explanation for this finding might be that in both cohorts of CMP patients, persistent avoidance behaviour, regardless of the triggering mechanism, resulted in deterioration of daily functioning. $A$ recent longitudinal study in children with G-HSD/hEDS showed that multi-systemic dysfunction was found to be the most important construct for functional impairment measured in terms of performance and capacity. Furthermore, an increase of pain, fatigue, and loss of postural control were related to disability over time. ${ }^{61}$

Regarding Health-Related Quality of life (HRQoL), cross-sectional studies in individuals with G-HSD/hEDS found lower levels of HRQoL compared to non-hypermobile pain-free peers. ${ }^{26,62-65}$ In addition, a longitudinal study showed that severely affected children with G-HSD/hEDS in terms of functional impairment had consistently lower HRQoL over the course of 3 years. ${ }^{61}$ In addition, poorer emotional functioning levels measured with the Pediatric Quality of Life Inventory were observed in children with G-HSD/hEDS compared to pain-free peers. ${ }^{66}$ Lower levels of HRQoL in children with G-HSD/hEDS were associated with pain ${ }^{63,65,66}$, fatigue ${ }^{64-66}$, and stress incontinence symptoms. ${ }^{66}$

In conclusion, disability and reduced quality of life are common in children and adults with G-HSD/hEDS. Pain, fatigue, reduced postural control, and multi-systemic disorders may potentially be important determinants of disability in individuals with G-HSD/hEDS.

\section{Discussion}

Many studies support the increased injury rate in adolescents with asymptomatic GJH and G-HSD and also pain experience in these adolescents is common. The psychological involvement of pain catastrophizing and pain-related fear is also known. As these factors might lead to avoidance behaviour, this is proposed to result in disuse, depression, 
disability, and reduced quality of life. Eventually, deconditioning may occur, further fuelling the vicious circle in adolescents with G-HSD/hEDS. Based on the evidence presented for the different components of the FAM it can be hypothesized that painrelated fear and GJH are important factors in the development and maintenance of CMP, eventually leading to disabling pain in adolescents with G-HSD/hEDS. According to this hypothesis, we would like to propose a FAM applied to adolescents with GHSD/hEDS (Figure 2.3).

Earlier studies showed that individuals with asymptomatic GJH and G-HSD/hEDS both have an increased risk of injuries ${ }^{18-20,22}$ and repetitive trauma, due to compromised structural integrity of connective tissue, might lead to (re)injury. ${ }^{21}$ In addition, individuals with G-HSD/hEDS experience more frequent pain ${ }^{23,24}$ and GJH represents a risk factor for developing musculoskeletal pain during adolescence. ${ }^{32,33}$ Therefore, it is hypothesized that being hypermobile makes you more vulnerable for developing CMP due to an increased risk of (re)injury and experiencing more frequent pain.

In addition it is proposed that individuals with G-HSD/hEDS have a vulnerability for heightened pain-related fear, which is confirmed in several studies. ${ }^{16,17,39}$ Literature also showed that more generalized anxiety is present in individuals with G-HSD/HEDS. ${ }^{67,68}$ Furthermore, an increased incidence of other psychiatric conditions, including panic disorders $^{58,69}$ and anxiety disorders ${ }^{67,68,70}$ were found to be associated with G-HSD/hEDS. It could even be hypothesized that the relationship between anxiety disorders and G-HSD/hEDS might also have a dysautonomic component, since several dysautonomia symptoms related to anxiety such as hyperventilation, nausea, and light-headedness were significantly more common in patients with G-HSD/hEDS. ${ }^{71}$ In addition, in adults with G-HSD/hEDS anxiety sensitivity is strongly associated with fearful appraisals of pain. ${ }^{72}$ Furthermore, a recent study in 9-year old children with asymptomatic GJH showed that high symptoms of GJH were associated with higher scores in anxiety symptoms. ${ }^{73}$ In conclusion, heightened pain-related fear and more generalized anxiety is common in individuals with G-HSD/hEDS. This might represent a general vulnerability for the cascade of cognitive/affective responses in the FAM.

It is now proposed that this vulnerability for heightened pain-related fear and anxiety causes avoidance behaviour. This avoidance behaviour seems to be a coping strategy to avoid (re)injury and to prevent musculoskeletal complaints. As a result, avoidance behaviour may lead to disability, depression, and disuse and eventually to physical deconditioning, further fuelling the vicious circle of CMP. It is hypothesized that physical deconditioning has additional negative consequences in individuals with G-HSD/hEDS. Their compensation mechanism, essential for joint stability to compensate for joint laxity, might fail. As a result of the failed compensation mechanism proprioception ${ }^{48,74-76}$ and balance ${ }^{44,77}$ can be impaired, which negatively influences body posture. An affected 
body posture, proprioceptive impairment, and decreased balance may lead to an increased risk for injury and affect gait patterns. ${ }^{77,78}$ This FAM applied to G-HSD/hEDS, describing a vulnerability for heightened pain-related fear and anxiety, might explain the higher prevalence of GJH in adolescents with functionally disabling CMP compared to the general population.

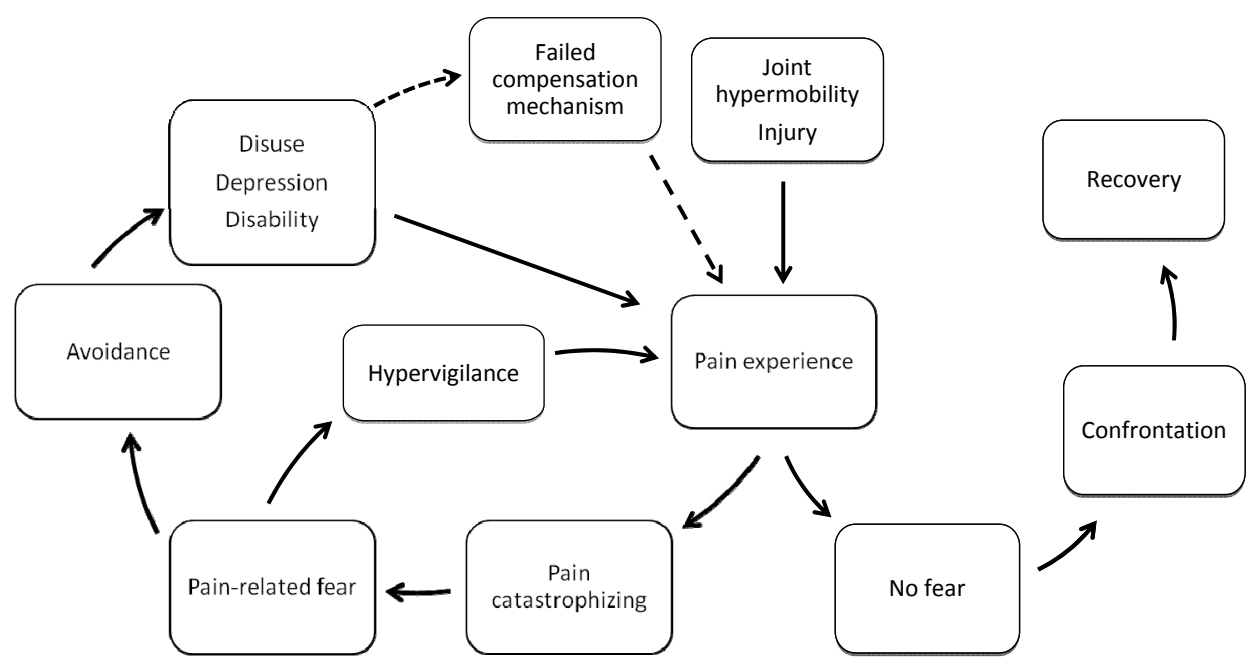

Figure 2.3 The adapted fear-avoidance model for adolescents with G-HSD/hEDS.

Currently there is some support in the literature for components of this hypothesized model, but are cross-sectional in nature, with only few evaluating more than one aspect of the FAM. We proposed that, both joint hypermobility and pain-related fear are important factors in the development and maintenance of CMP leading to disabling pain in adolescents with G-HSD/hEDS. Therefore, in explaining disability, it is important to focus on both the physical components related to joint hypermobility, in tandem with the psychological components such as pain-related fear, catastrophizing thoughts, and generalized anxiety in individuals with G-HSD/hEDS.

Future studies should further focus on identifying the underlying mechanism that causes functional impairment in adolescents with G-HSD/hEDS. A recent study already showed that children with G-HSD/hEDS who are severely affected have a high incidence of multisystemic complaints, in addition to high pain levels, fatigue, and poor postural control. ${ }^{61}$ Furthermore, there is a growing evidence of autonomic nervous system dysfunction in G-HSD/hEDS, including symptoms such as hypotension, orthostatic intolerance, presyncope, palpitations, postural orthostatic tachycardia, gastrointestinal dysfunction, and disturbed bladder function. ${ }^{71,79-82}$ Autonomic dysfunction contributes to disability 
and decreased quality of life in G-HSD/hEDS. ${ }^{79,81}$ In addition, increased autonomic dysfunctions were related to higher pain severity ${ }^{82,83}$ and autonomic dysfunction may result in increased interoceptive sensitivity. ${ }^{84}$ Higher pain severity in combination with increased interoceptive sensitivity may have additional emotional consequences, such as anxiety and fear, which also may contribute to higher disability levels. Furthermore, recent studies showed the potential role of neurodiversity, such as attentiondeficit/hyperactivity disorder (ADHD) and autism spectrum disorders (ASD) in GHSD/hEDS. ${ }^{85,86}$ The potential connection between these psychiatric disorders and GHSD/hEDS can be useful in clinical practice since it allows to better identify patients with ASD or ADHD who may be vulnerable for disabling pain. However, the causality between these disorders and G-HSD/hEDS remains questionable, due to the design of the studies. Despite the highly variable clinical presentation of G-HSD/hEDS the diagnosis is frequently overlooked and misdiagnosed. ${ }^{87}$ Therefore, in clinical practice and future studies these factors are important factors to take into account.

Furthermore, it is suggested to perform studies that evaluate the specific impact of hypermobility on catastrophizing, pain-related fear, hypervigilance, and avoidance behaviour, to further unravel the vulnerability for heightened pain-related fear and generalized anxiety. In addition, an experimental study may contribute to unravel the influence of both physiological (deconditioning) and psychological factors (pain-related fear) on physical performance in adolescents with G-HSD/hEDS. Verbunt et al. ${ }^{88}$ used an interpolated twitch technique during muscle testing. This technique alongside with surface EMG may distinguish the influence of both physiological and psychological factors during performance testing to confirm whether deconditioning or submaximal performance will influence physical test outcomes. Furthermore, it is important to examine associations over time, regarding physical and psychological functioning, in children with GJH before the onset of CMP to unravel the influence of GJH, physical, and psychological functioning in children who will or will not develop CMP.

\section{Conclusion}

This topical review integrates knowledge from different research fields of expertise in CMP, hypermobility related disorders, and rehabilitation medicine by presenting a FAM applied to adolescents with G-HSD/hEDS. It is proposed that both GJH and pain-related fear are important factors in the development and maintenance of CMP leading to disabling pain in adolescents with G-HSD/hEDS. Although scientific confirmation of this FAM applied to G-HSD/hEDS seems warranted, many studies are cross-sectional and 
Chapter 2

therefore the suggested mechanism should be interpreted with caution. Longitudinal studies are necessary to further unravel the mechanism of disabling G-HSD/hEDS. 


\section{References}

1. Perquin CW, Hazebroek-Kampschreur AA, Hunfeld JA, Bohnen AM, van Suijlekom-Smit LW, Passchier J, et al. Pain in children and adolescents: a common experience. Pain. 2000;87(1):51-8.

2. King S, Chambers CT, Huguet A, MacNevin RC, McGrath PJ, Parker L, et al. The epidemiology of chronic pain in children and adolescents revisited: a systematic review. Pain. 2011;152(12):2729-38.

3. Huguet A, Miro J. The severity of chronic pediatric pain: an epidemiological study. J Pain. 2008;9(3): 226-36.

4. Kashikar-Zuck S, Goldschneider KR, Powers SW, Vaught MH, Hershey AD. Depression and functional disability in chronic pediatric pain. Clin J Pain. 2001;17(4):341-9.

5. Jelsma LD, Geuze RH, Klerks MH, Niemeijer AS, Smits-Engelsman BC. The relationship between joint mobility and motor performance in children with and without the diagnosis of developmental coordination disorder. BMC Pediatr. 2013;13:35.

6. van Meulenbroek T, Huijnen IPJ, Wiertz CMH, Verbunt JA. Pain-Related Fear and Its Disabling Impact in Hypermobile Adolescents With Chronic Musculoskeletal Pain. J Orthop Sports Phys Ther. 2017;47(10): 775-81.

7. Grahame R, Bird HA, Child A. The revised (Brighton 1998) criteria for the diagnosis of benign joint hypermobility syndrome (BJHS). J Rheumatol. 2000;27(7):1777-9.

8. Remvig L, Engelbert RH, Berglund B, Bulbena A, Byers PH, Grahame R, et al. Need for a consensus on the methods by which to measure joint mobility and the definition of norms for hypermobility that reflect age, gender and ethnic-dependent variation: is revision of criteria for joint hypermobility syndrome and Ehlers-Danlos syndrome hypermobility type indicated? Rheumatology (Oxford). 2011;50(6):1169-71.

9. Castori M, Tinkle B, Levy H, Grahame R, Malfait F, Hakim A. A framework for the classification of joint hypermobility and related conditions. Am J Med Genet C Semin Med Genet. 2017;175(1):148-57.

10. Malfait F, Francomano C, Byers P, Belmont J, Berglund B, Black J, et al. The 2017 international classification of the Ehlers-Danlos syndromes. Am J Med Genet C Semin Med Genet. 2017;175(1):8-26.

11. Leeuw M, Goossens ME, Linton SJ, Crombez G, Boersma K, Vlaeyen JW. The fear-avoidance model of musculoskeletal pain: current state of scientific evidence. J Behav Med. 2007;30(1):77-94.

12. Vlaeyen JW, Linton SJ. Fear-avoidance and its consequences in chronic musculoskeletal pain: a state of the art. Pain. 2000;85(3):317-32.

13. Asmundson GJ, Noel M, Petter M, Parkerson HA. Pediatric fear-avoidance model of chronic pain: foundation, application and future directions. Pain Res Manag. 2012;17(6):397-405.

14. Simons LE, Kaczynski KJ. The Fear Avoidance model of chronic pain: examination for pediatric application. J Pain. 2012;13(9):827-35.

15. Goubert L, Simons LE. Cognitive styles and processes in paediatric pain. In: McGrath PA, Stevens BJ, Walker SM, T. ZW, editors. Oxford textbook paediatric Pain. Oxford: Oxford University Press; 2012.

16. Baeza-Velasco C, Bourdon C, Montalescot L, de Cazotte C, Pailhez G, Bulbena A, et al. Low- and highanxious hypermobile Ehlers-Danlos syndrome patients: comparison of psychosocial and health variables. Rheumatol Int. $2018 ; 38(5): 871-8$.

17. Celletti C, Castori M, La Torre G, Camerota F. Evaluation of kinesiophobia and its correlations with pain and fatigue in joint hypermobility syndrome/Ehlers-Danlos syndrome hypermobility type. Biomed Res Int. 2013;2013:580460.

18. Ruemper A, Watkins K. Correlations Between General Joint Hypermobility and Joint Hypermobility Syndrome and Injury in Contemporary Dance Students. J Dance Med Sci. 2012;16(4):161-6.

19. Russek LN, Errico DM. Prevalence, injury rate and, symptom frequency in generalized joint laxity and joint hypermobility syndrome in a "healthy" college population. Clin Rheumatol. 2016;35(4):1029-39.

20. Briggs J, McCormack M, Hakim AJ, Grahame R. Injury and joint hypermobility syndrome in ballet dancers-a 5-year follow-up. Rheumatology (Oxford). 2009;48(12):1613-4.

21. Ferrell WR, Tennant N, Sturrock RD, Ashton L, Creed G, Brydson G, et al. Amelioration of symptoms by enhancement of proprioception in patients with joint hypermobility syndrome. Arthritis Rheum. 2004; 50(10):3323-8. 
22. Pacey V, Nicholson LL, Adams RD, Munn J, Munns CF. Generalized joint hypermobility and risk of lower limb joint injury during sport: a systematic review with meta-analysis. Am J Sports Med. 2010;38(7): 1487-97.

23. Voermans NC, Knoop H, Bleijenberg G, van Engelen BG. Pain in ehlers-danlos syndrome is common, severe, and associated with functional impairment. J Pain Symptom Manage. 2010;40(3):370-8.

24. Castori M, Morlino S, Celletti C, Celli M, Morrone A, Colombi M, et al. Management of pain and fatigue in the joint hypermobility syndrome (a.k.a. Ehlers-Danlos syndrome, hypermobility type): principles and proposal for a multidisciplinary approach. Am J Med Genet A. 2012;158A(8):2055-70.

25. Adib N, Davies K, Grahame R, Woo P, Murray KJ. Joint hypermobility syndrome in childhood. A not so benign multisystem disorder? Rheumatology (Oxford). 2005;44(6):744-50.

26. Johannessen EC, Reiten HS, Lovaas H, Maeland S, Juul-Kristensen B. Shoulder function, pain and health related quality of life in adults with joint hypermobility syndrome/Ehlers-Danlos syndrome-hypermobility type. Disabil Rehabil. 2016;38(14):1382-90.

27. Voermans NC, Knoop $\mathrm{H}$. Both pain and fatigue are important possible determinants of disability in patients with the Ehlers-Danlos syndrome hypermobility type. Disabil Rehabil. 2011;33(8):706-7.

28. Pacey V, Adams RD, Tofts L, Munns CF, Nicholson LL. Joint hypermobility syndrome subclassification in paediatrics: a factor analytic approach. Arch Dis Child. 2015;100(1):8-13.

29. Scheper MC, Pacey V, Rombaut L, Adams RD, Tofts L, Calders P, et al. Generalized Hyperalgesia in Children and Adults Diagnosed With Hypermobility Syndrome and Ehlers-Danlos Syndrome Hypermobility Type: A Discriminative Analysis. Arthritis Care Res (Hoboken). 2017;69(3):421-9.

30. Di Stefano G, Celletti C, Baron R, Castori M, Di Franco M, La Cesa S, et al. Central sensitization as the mechanism underlying pain in joint hypermobility syndrome/Ehlers-Danlos syndrome, hypermobility type. Eur J Pain. 2016;20(8):1319-25.

31. English B. Neural and psychosocial mechanisms of pain sensitivity in fibromyalgia. Pain Manag Nurs. 2014;15(2):530-8.

32. Tobias JH, Deere K, Palmer S, Clark EM, Clinch J. Joint hypermobility is a risk factor for musculoskeletal pain during adolescence: findings of a prospective cohort study. Arthritis Rheum. 2013;65(4):1107-15.

33. Sohrbeck-Nohr O, Kristensen JH, Boyle E, Remvig L, Juul-Kristensen B. Generalized joint hypermobility in childhood is a possible risk for the development of joint pain in adolescence: a cohort study. BMC Pediatr. 2014;14:302.

34. El-Metwally A, Salminen JJ, Auvinen A, Kautiainen H, Mikkelsson M. Lower limb pain in a preadolescent population: prognosis and risk factors for chronicity--a prospective 1- and 4-year follow-up study. Pediatrics. 2005;116(3):673-81.

35. El-Metwally A, Salminen JJ, Auvinen A, Kautiainen H, Mikkelsson M. Risk factors for traumatic and nontraumatic lower limb pain among preadolescents: a population-based study of Finnish schoolchildren. BMC Musculoskelet Disord. 2006;7:3.

36. Goubert L, Crombez G, Van Damme S. The role of neuroticism, pain catastrophizing and pain-related fear in vigilance to pain: a structural equations approach. Pain. 2004;107(3):234-41.

37. Roelofs J, Peters ML, McCracken L, Vlaeyen JW. The pain vigilance and awareness questionnaire (PVAQ): further psychometric evaluation in fibromyalgia and other chronic pain syndromes. Pain. 2003; 101(3):299-306.

38. Rahman A, Daniel C, Grahame R. Efficacy of an out-patient pain management programme for people with joint hypermobility syndrome. Clin Rheumatol. 2014;33(11):1665-9.

39. Simmonds JV, Herbland A, Hakim A, Ninis N, Lever W, Aziz Q, et al. Exercise beliefs and behaviours of individuals with Joint Hypermobility syndrome/Ehlers-Danlos syndrome - hypermobility type. Disabil Rehabil. 2017:1-11.

40. Schmidt A, Corcoran K, Grahame R, de CWAC. How do people with chronically painful joint hypermobility syndrome make decisions about activity? Br J Pain. 2015;9(3):157-66.

41. Van Meulenbroek T, Huijnen I, Stappers N, Engelbert R, Verbunt J. Generalized joint hypermobility and perceived harmfulness in healthy adolescents; impact on muscle strength, motor performance and physical activity level. Physiother Theory Pract. 2020:1-10.

42. Kanfer F, Philips J. Learning foundations of behavior therapy. New York: John Wiley \& Son; 1970.

43. Linton SJ, Vlaeyen J, Ostelo R. The back pain beliefs of health care providers: are we fear-avoidant? J Occup Rehabil. 2002;12(4):223-32. 
44. Schubert-Hjalmarsson E, Ohman A, Kyllerman M, Beckung E. Pain, balance, activity, and participation in children with hypermobility syndrome. Pediatr Phys Ther. 2012;24(4):339-44.

45. Scheper M, de Vries J, Beelen A, de Vos R, Nollet F, Engelbert R. Generalized joint hypermobility, muscle strength and physical function in healthy adolescents and young adults. Curr Rheumatol Rev. 2014; 10(2):117-25.

46. Verbunt JA, Seelen HA, Vlaeyen JW, van de Heijden GJ, Heuts PH, Pons K, et al. Disuse and deconditioning in chronic low back pain: concepts and hypotheses on contributing mechanisms. Eur J Pain. 2003;7(1): 9-21.

47. Rombaut L, Malfait F, De Wandele I, Taes Y, Thijs Y, De Paepe A, et al. Muscle mass, muscle strength, functional performance, and physical impairment in women with the hypermobility type of Ehlers-Danlos syndrome. Arthritis Care Res (Hoboken). 2012;64(10):1584-92.

48. Fatoye F, Palmer S, Macmillan F, Rowe P, van der Linden M. Proprioception and muscle torque deficits in children with hypermobility syndrome. Rheumatology (Oxford). 2009;48(2):152-7.

49. Scheper M, Rombaut L, de Vries J, De Wandele I, van der Esch M, Visser B, et al. The association between muscle strength and activity limitations in patients with the hypermobility type of Ehlers-Danlos syndrome: the impact of proprioception. Disabil Rehabil. 2017;39(14):1391-7.

50. Huijnen IPJ, Verbunt J, Wittink HM, Smeets RJEM. Physical performance measurement in chronic low back pain: measuring physical capacity of pain-related behaviour? European Journal of Physiotherapy. 2013;15(3):103-10.

51. Engelbert RH, van Bergen M, Henneken T, Helders PJ, Takken T. Exercise tolerance in children and adolescents with musculoskeletal pain in joint hypermobility and joint hypomobility syndrome. Pediatrics. 2006;118(3):e690-6.

52. Hanewinkel-van Kleef YB, Helders PJ, Takken T, Engelbert RH. Motor performance in children with generalized hypermobility: the influence of muscle strength and exercise capacity. Pediatr Phys Ther. 2009;21(2):194-200.

53. Jindal P, Narayan A, Ganesan S, MacDermid JC. Muscle strength differences in healthy young adults with and without generalized joint hypermobility: a cross-sectional study. BMC Sports Sci Med Rehabil. 2016;8:12.

54. Scheper MC, de Vries JE, de Vos R, Verbunt J, Nollet F, Engelbert RH. Generalized joint hypermobility in professional dancers: a sign of talent or vulnerability? Rheumatology (Oxford). 2013;52(4):651-8.

55. Jensen BR, Olesen AT, Pedersen MT, Kristensen JH, Remvig L, Simonsen EB, et al. Effect of generalized joint hypermobility on knee function and muscle activation in children and adults. Muscle Nerve. 2013;48(5):762-9.

56. Juul-Kristensen B, Hansen $\mathrm{H}$, Simonsen EB, Alkjaer T, Kristensen JH, Jensen BR, et al. Knee function in 10year-old children and adults with Generalised Joint Hypermobility. Knee. 2012;19(6):773-8.

57. American Psychiatric Association K. Diagnostic and statistical manual of mental disorders: DSM-5. 5th ed. Washington: American Psychiatric Association Publishing; 2013.

58. Smith TO, Easton V, Bacon H, Jerman E, Armon K, Poland F, et al. The relationship between benign joint hypermobility syndrome and psychological distress: a systematic review and meta-analysis. Rheumatology (Oxford). 2014;53(1):114-22.

59. Cederlof M, Larsson H, Lichtenstein P, Almqvist C, Serlachius E, Ludvigsson JF. Nationwide populationbased cohort study of psychiatric disorders in individuals with Ehlers-Danlos syndrome or hypermobility syndrome and their siblings. BMC Psychiatry. 2016;16:207.

60. Scheper MC, Juul-Kristensen B, Rombaut L, Rameckers EA, Verbunt J, Engelbert RH. Disability in Adolescents and Adults Diagnosed With Hypermobility-Related Disorders: A Meta-Analysis. Arch Phys Med Rehabil. 2016;97(12):2174-87.

61. Scheper MC, Nicholson LL, Adams RD, Tofts L, Pacey V. The natural history of children with joint hypermobility syndrome and Ehlers-Danlos hypermobility type: a longitudinal cohort study. Rheumatology (Oxford). 2017;56(12):2073-83.

62. Rombaut L, Malfait F, Cools A, De Paepe A, Calders P. Musculoskeletal complaints, physical activity and health-related quality of life among patients with the Ehlers-Danlos syndrome hypermobility type. Disabil Rehabil. 2010;32(16):1339-45.

63. Fatoye F, Palmer S, Macmillan F, Rowe P, van der Linden M. Pain intensity and quality of life perception in children with hypermobility syndrome. Rheumatol Int. 2012;32(5):1277-84. 
64. Zekry OA, Ahmed MA, Elwahid HAEA. The impact of fatigue on health related quality of life in adolescents with benign joint hypermobility syndrome. Egypt Rheumatol. 2013;35:77-85.

65. Mu W, Muriello M, Clemens JL, Wang Y, Smith CH, Tran PT, et al. Factors affecting quality of life in children and adolescents with hypermobile Ehlers-Danlos syndrome/hypermobility spectrum disorders. Am J Med Genet A. 2019;179(4):561-9.

66. Pacey V, Tofts L, Adams RD, Munns CF, Nicholson LL. Quality of life prediction in children with joint hypermobility syndrome. J Paediatr Child Health. 2015;51(7):689-95.

67. Bulbena A, Gago J, Pailhez G, Sperry L, Fullana MA, Vilarroya O. Joint hypermobility syndrome is a risk factor trait for anxiety disorders: a 15-year follow-up cohort study. Gen Hosp Psychiatry. 2011;33(4): 363-70.

68. Bulbena-Cabre A, Duno L, Almeda S, Batlle S, Camprodon-Rosanas E, Martin-Lopez LM, et al. Joint hypermobility is a marker for anxiety in children. Rev Psiquiatr Salud Ment. 2019;12(2):68-76.

69. Garcia Campayo J, Asso E, Alda M, Andres EM, Sobradiel N. Association between joint hypermobility syndrome and panic disorder: a case-control study. Psychosomatics. 2010;51(1):55-61.

70. Javadi Parvaneh V, Modaress S, Zahed G, Rahmani K, Shiari R. Prevalence of generalized joint hypermobility in children with anxiety disorders. BMC Musculoskelet Disord. 2020;21(1):337.

71. Gazit Y, Nahir AM, Grahame R, Jacob G. Dysautonomia in the joint hypermobility syndrome. Am J Med. 2003;115(1):33-40.

72. Ocanez KL, McHugh RK, Otto MW. A meta-analytic review of the association between anxiety sensitivity and pain. Depress Anxiety. 2010;27(8):760-7.

73. Ezpeleta L, Navarro JB, Osa N, Penelo E, Bulbena A. Joint Hypermobility Classes in 9-Year-Old Children from the General Population and Anxiety Symptoms. J Dev Behav Pediatr. 2018;39(6):481-8.

74. Sahin N, Baskent A, Cakmak A, Salli A, Ugurlu H, Berker E. Evaluation of knee proprioception and effects of proprioception exercise in patients with benign joint hypermobility syndrome. Rheumatol Int. 2008; 28(10):995-1000.

75. Rombaut L, De Paepe A, Malfait F, Cools A, Calders P. Joint position sense and vibratory perception sense in patients with Ehlers-Danlos syndrome type III (hypermobility type). Clin Rheumatol. 2010;29(3):289-95.

76. Smith TO, Jerman E, Easton V, Bacon H, Armon K, Poland F, et al. Do people with benign joint hypermobility syndrome (BJHS) have reduced joint proprioception? A systematic review and metaanalysis. Rheumatol Int. 2013;33(11):2709-16.

77. Rombaut L, Malfait F, De Wandele I, Thijs Y, Palmans T, De Paepe A, et al. Balance, gait, falls, and fear of falling in women with the hypermobility type of Ehlers-Danlos syndrome. Arthritis Care Res (Hoboken). 2011;63(10):1432-9.

78. Scheper MC, de Vries JE, Verbunt J, Engelbert RH. Chronic pain in hypermobility syndrome and EhlersDanlos syndrome (hypermobility type): it is a challenge. J Pain Res. 2015;8:591-601.

79. Hakim A, O'Callaghan C, De Wandele I, Stiles L, Pocinki A, Rowe P. Cardiovascular autonomic dysfunction in Ehlers-Danlos syndrome-Hypermobile type. Am J Med Genet C Semin Med Genet. 2017;175(1):168-74.

80. Hakim AJ, Grahame R. Non-musculoskeletal symptoms in joint hypermobility syndrome. Indirect evidence for autonomic dysfunction? Rheumatology (Oxford). 2004;43(9):1194-5.

81. Fikree A, Chelimsky G, Collins H, Kovacic K, Aziz Q. Gastrointestinal involvement in the Ehlers-Danlos syndromes. Am J Med Genet C Semin Med Genet. 2017;175(1):181-7.

82. De Wandele I, Rombaut L, Leybaert L, Van de Borne P, De Backer T, Malfait F, et al. Dysautonomia and its underlying mechanisms in the hypermobility type of Ehlers-Danlos syndrome. Semin Arthritis Rheum. 2014;44(1):93-100.

83. De Wandele I, Calders P, Peersman W, Rimbaut S, De Backer T, Malfait F, et al. Autonomic symptom burden in the hypermobility type of Ehlers-Danlos syndrome: a comparative study with two other EDS types, fibromyalgia, and healthy controls. Semin Arthritis Rheum. 2014;44(3):353-61.

84. Mallorqui-Bague N, Garfinkel SN, Engels M, Eccles JA, Pailhez G, Bulbena A, et al. Neuroimaging and psychophysiological investigation of the link between anxiety, enhanced affective reactivity and interoception in people with joint hypermobility. Front Psychol. 2014;5:1162.

85. Baeza-Velasco C, Cohen D, Hamonet C, Vlamynck E, Diaz L, Cravero C, et al. Autism, Joint HypermobilityRelated Disorders and Pain. Front Psychiatry. 2018;9:656. 
86. Baeza-Velasco C, Sinibaldi L, Castori M. Attention-deficit/hyperactivity disorder, joint hypermobilityrelated disorders and pain: expanding body-mind connections to the developmental age. Atten Defic Hyperact Disord. 2018;10(3):163-75.

87. Grahame R, Hakim AJ. Hypermobility. Curr Opin Rheumatol. 2008;20(1):106-10.

88. Verbunt JA, Seelen HA, Vlaeyen JW, Bousema EJ, van der Heijden GJ, Heuts PH, et al. Pain-related factors contributing to muscle inhibition in patients with chronic low back pain: an experimental investigation based on superimposed electrical stimulation. Clin J Pain. 2005;21(3):232-40. 



\section{Chapter 3}

Pain-related fear and its disabling impact in hypermobile adolescents with chronic musculoskeletal pain

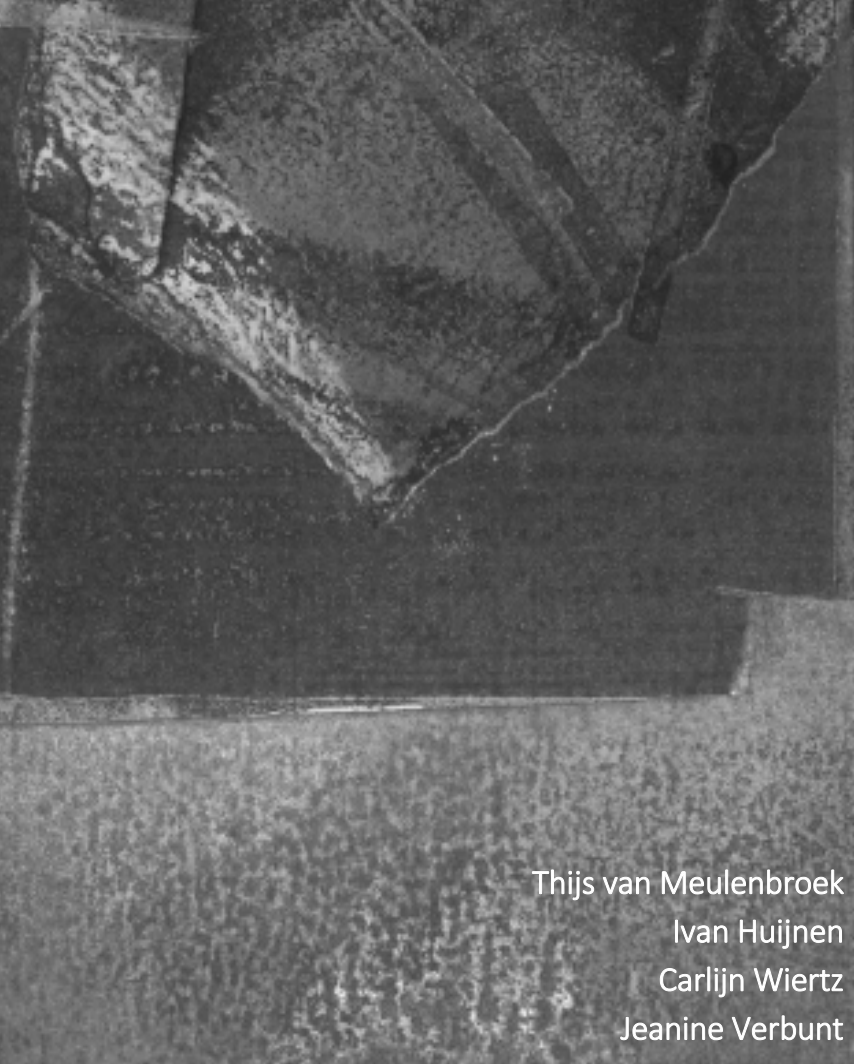
Journal of Orthopaedic \& Sports Physical Therapy. 2017;47 (10):775-781 


\section{Abstract}

\section{Study design}

Cross-sectional study.

\section{Background}

Chronic musculoskeletal pain (CMP) has a negative impact on physical functioning. During adolescence, joint hypermobility is a potential risk factor for developing CMP, and pain-related fear contributes to the persistence of CMP. Whether pain-related fear and hypermobility are related, and even reinforce each other, resulting in a stronger association with perceived level of disability, is still unknown.

\section{Objectives}

To evaluate whether pain-related fear has a stronger association with disability in hypermobile compared to non-hypermobile adolescents with CMP.

\section{Methods}

The study included 116 adolescents with CMP. The presence of hypermobility was assessed using the Beighton score. Measures of pain intensity, age, sex, and pain-related fear were collected and included in the multivariable model. Hierarchical regression analysis, with disability as the dependent variable, was used to examine the interaction between hypermobility and pain-related fear.

\section{Results}

Hypermobile adolescents with CMP do not have more pain-related fear compared to non-hypermobile adolescents with CMP. There was no interaction effect between hypermobility and pain-related fear in explaining disability $(\beta=0.20, p=0.42)$. Similarly, perceived harmfulness of balance-related activities was not more strongly associated with disability in hypermobile adolescents with CMP.

\section{Conclusion}

The association of pain-related fear with the perceived level of disability is not more pronounced in hypermobile compared to non-hypermobile adolescents with CMP. 


\section{Introduction}

Chronic pain is a common occurrence among adolescents. Up to $25 \%$ of all schoolchildren and adolescents, report chronic pain. ${ }^{1,2}$ Along with abdominal pain and headache, chronic musculoskeletal pain (CMP) is one of the most frequently reported pain complaints in adolescents. ${ }^{1,3}$ In adolescents with CMP, pain complaints without a specific diagnosis often relate to muscular, ligamentous, bony or joint structures and last for longer than 3 months. ${ }^{4}$ The incidence of CMP is reported to be higher among girls ${ }^{1,5}$ and increases with age during adolescence. ${ }^{1}$ In approximately $40 \%$ of adolescents with

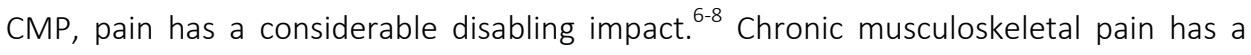
negative impact on physical functioning, mood, social functioning, and family, reducing the adolescent's quality of life. ${ }^{4,6,9-11}$ In adolescents with CMP, higher pain intensity has been shown to be associated with greater disability. ${ }^{12,13}$

Despite a broad array of medical diagnoses, only $10 \%$ to $30 \%$ of pain complaints can be explained based on a specific somatic disease. ${ }^{14,15}$ Because there is not always a somatic solution to explain CMP, a biopsychosocial approach to the management of CMP should be considered. ${ }^{4}$ In adults, the fear-avoidance model has been proposed and shown to explain the disabling role of pain-related fear. ${ }^{16-18}$ This model posits that catastrophizing thoughts may lead to the development of pain-related fear, thus leading to avoidance of feared activities. Over the long term, this avoidance behaviour may result in disability, depression, and disuse, further fuelling the vicious circle of disabling CMP. ${ }^{19-22}$ Recently, more evidence has become available to confirm that this model can be applied to explain pain-related disability in the paediatric and adolescent populations. ${ }^{23,24}$

Recent evidence has also shown that hypermobility is a risk factor for developing CMP. ${ }^{25,26}$ Generalized joint hypermobility $(\mathrm{GJH})$ is characterized by generalized joint laxity and increased joint mobility. In children with CMP the prevalence of GJH is $40 \%$ to $48 \%{ }^{27-29}$ This number far exceeds the prevalence of GJH in the general child population (19.2\%). ${ }^{30}$ Generalized joint hypermobility associated with pain and symptoms such as arthralgia, soft tissue injury, and joint instability is referred to as joint hypermobility syndrome. Studies in children and adolescents showed that joint hypermobility syndrome is characterized by decreased muscle strength ${ }^{31}$, impaired proprioception ${ }^{31}$, disabling fatigue ${ }^{32}$, reduced balance ${ }^{33}$, and problems with gait, falls, and coordination. ${ }^{34}$ In adults, an increased incidence of psychiatric conditions, including anxiety disorders $^{35,36}$, panic disorders ${ }^{37,38}$, and depression ${ }^{38,39}$ was also found to be associated with joint hypermobility syndrome.

A study by Scheper et al. ${ }^{40}$ has shown that young healthy adults with GJH tend to avoid sports and outdoor activities and to prefer more stable activities like cycling. In view of their sense of impaired balance, this avoidance behaviour is hypothesized to be a coping 
strategy to avoid joint luxation or injury and thus prevent the development of musculoskeletal complaints. Therefore, it could be assumed that pain-related fear and, more specifically, the perceived harmfulness of balance-related activities are more pronounced in hypermobile adolescents with CMP compared to adolescents with CMP who have no hypermobility, possibly resulting in avoidance behaviour leading to impaired daily functioning. In the long term, impaired daily functioning may also result in a further decrease in muscle strength. For hypermobile adolescents with CMP, loss of muscle strength might act to further reduce joint stability, resulting in a higher risk of activities such as walking, and problems such as falls and poor coordination, causing the onset of pain. This might explain the higher prevalence of hypermobility in adolescents with $\mathrm{CMP}^{27-29}$ compared to the general adolescent population. ${ }^{30}$ Whether hypermobile adolescents with CMP have more pain-related fear compared to non-hypermobile adolescents with CMP and whether pain-related fear and being hypermobile reinforce each other, resulting in a stronger association with perceived disability, are still unknown.

Therefore, the aim of this study was to evaluate whether pain-related fear has a stronger association with disability in hypermobile compared to non-hypermobile adolescents with CMP. In addition, we explored whether the perceived harmfulness of balancerelated activities had a stronger association with disability in hypermobile compared to non-hypermobile adolescents with CMP.

\section{Methods}

\section{Participants}

Adolescents (11 to 21 years of age) with CMP, who were referred to the university outpatient rehabilitation clinic specialised in pain rehabilitation (Adelante/Maastricht University Medical Center+, Maastricht, the Netherlands) between June 2012 and December 2015, were included in this cross-sectional study. Inclusion criteria were (1) non-specific musculoskeletal pain for longer than 3 months, (2) not diagnosed with a specific somatic disorder that could explain the current pain problem (e.g., neurological, orthopaedic or rheumatoid disorders), and (3) sufficient proficiency in the Dutch language to complete the questionnaires.

The age range of 11 to 21 years was consistent with the definition of adolescents by the American Academy of Pediatrics, ${ }^{41}$ which defines adolescence as including 3 stages: early adolescence, 11 to 14 years of age; middle adolescence, 15 to 17 years of age; and late adolescence, 18 to 21 years of age. 


\section{Procedure}

Adolescents were referred to the university outpatient rehabilitation clinic for a consultation with a physician in rehabilitation medicine. All patients were asked to complete web-based questionnaires. Questionnaires were part of the regular health care procedure. Patients were asked whether their data could be used anonymously for research purposes. If they agreed, the data were included in the research database. Ethical approval for this protocol was granted by the Medical Ethics Committee of the Academic Hospital Maastricht/Maastricht University, the Netherlands (NL41712.068.12/ METC 12-3-052). The set of questionnaires retrieved information on sociodemographic variables (age, sex, education level, and school absences), duration of pain, pain intensity, pain catastrophizing, functional disability, and fear of pain. A physician in rehabilitation medicine or a physical therapist assessed hypermobility using the Beighton score.

A subgroup of all participating adolescents received multidisciplinary rehabilitation treatment. In this group, the perceived harmfulness of activities was measured during the first session of the treatment programme, at which time the patients had not yet received any educational instructions or multidisciplinary treatment.

\section{Measures}

\section{Hypermobility}

The presence of hypermobility was assessed using the Beighton score, which consists of 5 functional tests, with a maximum score of 9 points (Table 3.1). The standardized Beighton protocol is a valid instrument for the evaluation of generalized joint mobility in elementary school children. ${ }^{42}$ The full protocol for testing hypermobility is described by Smits-Engelsman et al. ${ }^{42}$ In the current study, this assessment was performed by a clinician. Based on the result of this assessment, the patients were assigned to 1 of 2 groups: patients who had a Beighton score of 5 or greater were assigned to the group with CMP and hypermobility, and patients who had a Beighton score of less than 5 were assigned to the group with CMP with no hypermobility. ${ }^{43}$

Table 3.1 Beighton criteria for joint hypermobility.

\begin{tabular}{lcc}
\hline Joint examination & Points & Criteria for positive sign \\
\hline Passive hyperextension of the small finger (measured bilaterally) & 2 & $>90^{\circ}$ \\
Passive thumb apposition to the forearm (measured bilaterally) & 2 & Thumb touches forearm \\
Elbow hyperextension (each elbow) & 2 & $>10^{\circ}$ \\
Knee hyperextension (each knee) & 2 & $>10^{\circ}$ \\
Standing trunk flexion with knees fully extended & 1 & Both palms flat on floor \\
\hline
\end{tabular}




\section{Sociodemographic variables}

The following sociodemographic data were collected: age, sex, education level, duration of pain, school absences during the last year, and pain intensity. Pain intensity was measured using a 100-mm visual analogue scale (VAS), ranging from 0 (no pain) to 100 (worst pain imaginable). The participants rated their pain intensity (1) at the moment the questionnaire was completed, (2) for the worst/most severe pain experienced in the last week, and (3) for the least pain experienced in the last week. The mean of these 3 VAS scores was used in the analyses. The VAS seems to be reliable and valid in children older than 8 years. ${ }^{44,45}$

\section{Functional disability}

Functional disability was measured using the Functional Disability Inventory (FDI). The FDI is a questionnaire for children and adolescents that measures the perceived difficulty of performing activities at home, at school, and in recreational and social domains. It contains 15 rated on a 5-point scale ranging from 0 (no trouble) to 4 (impossible). The total score on the FDI ranges from 0 to 60 , with a higher score indicating greater painrelated disability. The reliability and validity of the FDI appear to be sufficient for assessing pain-related disabilities in children and adolescents. ${ }^{46,47}$

\section{Fear of pain}

The Fear of Pain Questionnaire-Child report (FOPQ-C) was used to measure pain-related fear in children and adolescents with chronic pain. It contains 24 items and 2 scales, fear of pain and avoidance of activities. Each item is scored on a 5-point scale, ranging from 0 (strongly disagree) to 4 (strongly agree), and the total scores ranges from 0 to 96 , with higher scores indicate higher levels of pain-related fear and avoidance. The FOPQ-C appears has been shown to be a valid and reliable. ${ }^{48}$

\section{Perceived harmfulness}

The Photograph Series of Daily Activities for youth (PHODA-youth) was used to measure perceived harmfulness, or the indication to what extent the patient perceives the activity to be harmful for the painful body part. The PHODA-youth contains photographs of 51 age-specific daily life activities and social situations that are scored on the level of perceived harmfulness of performing these activities. The PHODA-youth consists of 3 categories: daily life and household activities, intensive physical activities, and social activities. Adolescents rate the photographs from 0 (completely harmless) to 10 (extremely harmful). Subscale scores for the 3 categories were obtained by summing 
the scores for all items in that subscale. To obtain a total score, all items were summed. The electronic version of PHODA-youth has been found to be valid and reliable. ${ }^{49}$ For the current study, a subscale of the perceived harmfulness of balance-related activities (PHODA-balance) was developed. Two authors, a physical therapist (T.M.) and a physiatrist (J.V.), independently scored all 51 age-specific daily life activities and social situations as yes or no, depending on their relation to balance. If both authors marked an activity as yes, then the activity was added to the PHODA-balance. In instances of disagreement, a third author (I.H.), a physical therapist, made the final decision whether to include the activity in the PHODA-balance. Seven activities were selected as activities related to balance, 6 from the PHODA physical activities subscale and 1 from the PHODA daily life and household activities subscale. To obtain a subscale score on the PHODAbalance, scores on the 7 items were summed, as are scores on the traditional subscales. The selected activities were: (item 5) putting pants on, (item 20) participating in physical exercise/gym, (item 28) dancing/sports, (item 30) running, (item 32) kicking a ball, (item 35) jumping over something, and (item 36) balancing on 1 leg.

\section{Pain catastrophizing}

The Pain Catastrophizing Scale for Children (PCS-C) measures catastrophizing thoughts about pain. It contains 13 items describing different thoughts and feelings that children and adolescents may experience when they are in pain, each rated on a 5-point scale ranging from 0 (not at all) to 4 (extremely). The total score on the PCS-C can range from 0 to 52 , with higher score indicating more catastrophizing thoughts about pain. The PCS$C$ has been reported to be reliable and valid for children and adolescents. ${ }^{50}$

\section{Statistical analysis}

The results were tested for normality using histograms and the Kolmogorov-Smirnov goodness-of-fit test. Descriptive data for the hypermobility and no-hypermobility groups were calculated. Where distribution of the data was normal, continuous data were shown as mean $\pm S D$; where the distribution was non normal, the data were shown as median (interquartile range); and categorical data were presented as absolute frequencies or percentages. Differences between the hypermobility and nohypermobility groups were analyzed using an independent sample $t$ test, the MannWhitney $U$ test, the Kruskall-Wallis test, and the chi-square test.

To test the hypothesis that pain-related fear would have a stronger association with disability in adolescents in the hypermobility group compared to those in the nohypermobility group, a hierarchical (blockwise entry) regression analysis was performed. Variables were entered in the regression model in 3 blocks. With the FDI as the 
dependent variable in the model, VAS, age, and sex (female=0; male=1) were introduced as independent variables in the first step to control for demographic variables and pain intensity. In the second step, pain-related fear (FOPQ-C) and the group variable of hypermobility (hypermobility/no-hypermobility; dichotomous variable) were entered simultaneously. Finally, the interaction term (FOPQ-C $x$ hypermobility/no-hypermobility) was introduced in the third step. In case of a significant interaction, additional regression analyses were performed for hypermobility and no-hypermobility groups separately to identify influencing factors for disability.

First, the Pearson correlation between FOPQ-C and PHODA-balance was calculated. The regression analysis was then repeated with $\mathrm{PHODA}$-balance instead of $\mathrm{FOPQ}-\mathrm{C}$ in the model, to explore whether the perceived harmfulness of balance-related activities had a stronger association with disability in hypermobile compared to non-hypermobile adolescents with CMP. For all regression analyses, a collinearity check was performed. Collinearity was considered a problem if the variance inflation factor was above $10 .^{51}$ The alpha level was set at 0.05. All statistical analyses were performed in IBM SPSS Statistics for Windows, version 23.0 (IBM Corp, Armonk, NY, USA).

\section{Results}

\section{Descriptive analyses}

A total of 183 adolescents (162 female, 21 male) were asked if their data could be used anonymously for research purposes, and data from 116 adolescents were included in the current study. Sixty-seven adolescents were excluded from the analyses for the following reasons: 8 ultimately proved to have a specific somatic disorder explaining the current pain problem, 38 did not complete the questionnaires, and the Beighton scores of 21 potential participants were unknown. Of the 116 adolescents, only 45 received multidisciplinary treatment, and, for this reason, the PHODA-youth was only measured in this subgroup (Figure 3.1).

The characteristics of each group are presented in Table 3.2. No significant differences were found between the hypermobility group and the no-hypermobility group with respect to sociodemographic variables, such as age, sex, education level, and school absences. The duration of pain, VAS, PCS-C, FDI, FOPQ-C, and all PHODA outcomes also did not significantly differ between groups. 
Pain-related fear and its disabling impact in hypermobile adolescents with chronic musculoskeletal pain

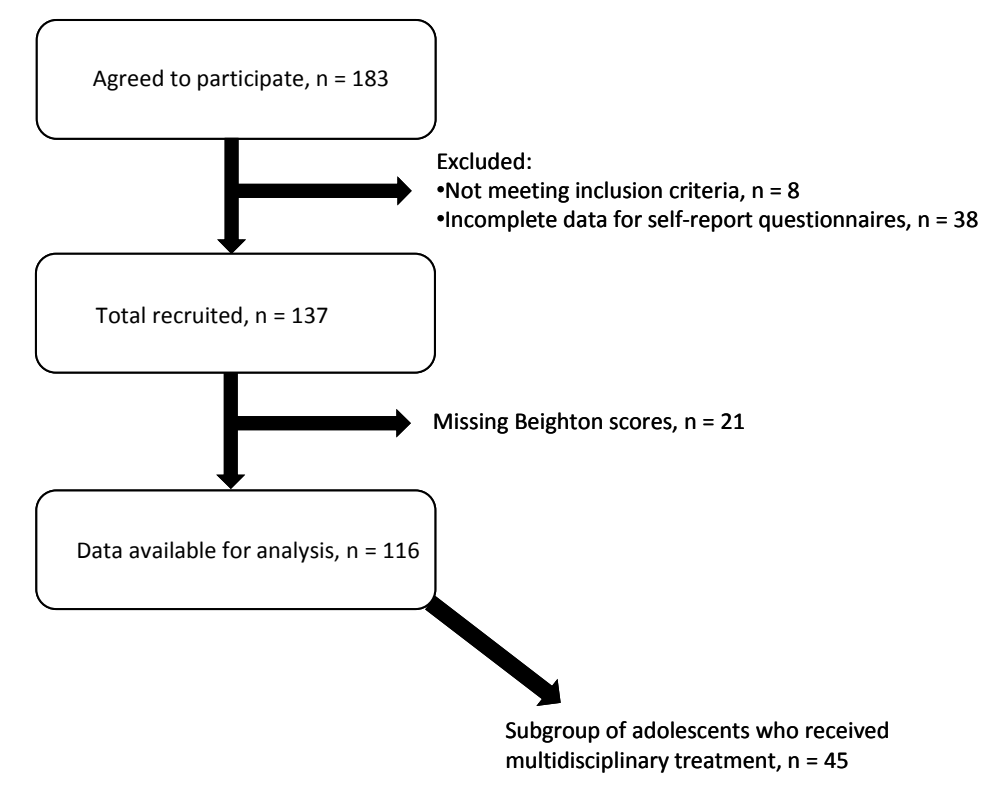

Figure 3.1 Flow chart representing the data of patients who were included in the current study.

\section{The association between pain-related fear, hypermobility, and disability}

Table 3.3 presents the outcome of the regression analysis with disability as the dependent variable. The inclusion of VAS, age, and sex as independent variables in the first step showed that no significant association with disability was found. In the second step, the FOPQ-C and the group variable of hypermobility/no hypermobility were added to the model, resulting in a significant model $\left(R^{2}=0.32\right.$, adjusted $\left.R^{2}=0.29, p<0.001\right)$. Only the FOPQ-C was associated with disability $(\beta=0.56, p<0.001)$, indicating that a higher level of pain-related fear was associated with more disabilities. In the third step, the interaction term (FOPQ-C $x$ hypermobility/no-hypermobility) was added in the model, but it did not appear to be significantly associated with disability. 
Table 3.2 Characteristics of the study sample of adolescents with chronic musculoskeletal pain.*

\begin{tabular}{|c|c|c|c|}
\hline & HM group $(n=40)$ & NHM group $(n=76)$ & Test statistics \\
\hline Sex (female/male) & $36 / 4$ & $67 / 9$ & $\chi_{1}^{2}=0.09, p=0.52$ \\
\hline Age, $y^{a}$ & $16.0 \pm 2.4$ & $16.1 \pm 2.4$ & $\begin{array}{c}t_{114}=0.23 \\
(-0.82,1.04)\end{array}$ \\
\hline Education level, $n(\%)^{b}$ & & & $\mathrm{H}_{1}=0.01, p=0.93$ \\
\hline Primary school & $2(5)$ & $5(6.6)$ & \\
\hline LGSE & $10(25)$ & $22(28.9)$ & \\
\hline HGSE & $11(27.5)$ & $17(22.4)$ & \\
\hline Pre-university education & $7(17.5)$ & $10(13.2)$ & \\
\hline Vocational Education & $9(22.5)$ & $17(22.4)$ & \\
\hline University of Applied Sciences & $1(2.5)$ & $4(5.3)$ & \\
\hline University & $0(0)$ & $1(1.3)$ & \\
\hline Duration of pain, $n(\%)^{b}$ & & & $\mathrm{H}_{1}=0.59, p=0.44$ \\
\hline 3-6 months & $3(7.9)$ & $7(10.0)$ & \\
\hline 6-12 months & $10(26.3)$ & $20(28.6)$ & \\
\hline $1-5$ years & $20(52.6)$ & $38(54.3)$ & \\
\hline$>5$ years & $5(13.2)$ & $5(7.1)$ & \\
\hline School absences, $\mathrm{n}(\%)^{\mathrm{b}}$ & & & $\mathrm{H}_{1}=0.15, p=0.70$ \\
\hline $0-14$ days & $20(51.3)$ & $39(52.7)$ & \\
\hline $15-30$ days & $8(20.5)$ & $9(12.2)$ & \\
\hline 1-3 months & $6(15.4)$ & $11(14.9)$ & \\
\hline 4-6 months & $3(7.7)$ & $6(8.1)$ & \\
\hline 7-12 months & $2(5.1)$ & $9(12.2)$ & \\
\hline VAS $^{a}$ & $59.0 \pm 17.1$ & $56.8 \pm 18.3$ & $t_{99}=-0.58(-9.60,5.30)$ \\
\hline $\mathrm{FDI}^{\mathrm{a}}$ & $22.4 \pm 10.2$ & $23.7 \pm 9.7$ & $\mathrm{t}_{114}=0.69(-2.51,5.15)$ \\
\hline FOPQ- $C^{a}$ & $39.4 \pm 17.1$ & $39.1 \pm 14.1$ & $t_{114}=-0.09(-6.14,5.61)$ \\
\hline PCS- $C^{b}$ & $22.8(12.0-31.8)$ & $20.5(12.3-28.3)$ & $\begin{array}{c}\mathrm{U}=1336, z=-1.07 \\
p=0.29\end{array}$ \\
\hline PHODA-youth total ${ }^{a, c}$ & $190.6 \pm 83.2$ & $154.1 \pm 97.1$ & $t_{43}=-1.22(-96.80,24.10)$ \\
\hline PHODA ADL subscale ${ }^{a, c}$ & $26.3(9.0-49.6)$ & $11.5(1.5-31.0)$ & $\begin{array}{c}\mathrm{U}=143, z=-1.82 \\
p=0.07\end{array}$ \\
\hline PHODA PA subscale & $126.8 \pm 47.3$ & $113.6 \pm 60.2$ & $t_{43}=-0.72(-50.00,23.60)$ \\
\hline PHODA SA subscale & $30.3 \pm 22.0$ & $22.2 \pm 21.6$ & $t_{43}=-1.16(-22.20,6.00)$ \\
\hline PHODA-balance ${ }^{a, c}$ & $33.0 \pm 12.9$ & $28.6 \pm 16.3$ & $t_{43}=-0.90(-14.40,5.50)$ \\
\hline
\end{tabular}

Abbreviations: $A D L=$ subscore daily life activities; $F D I=$ Functional Disability Inventory; $F O P Q-C=$ Fear of Pain Questionnaire-Child report; HGSE = higher general secondary education; HM = hypermobility; LGSE = lower general secondary education; $\mathrm{NHM}=$ no hypermobility; $\mathrm{PA}=$ subscore physical activities; $\mathrm{PCS}-\mathrm{C}=$ Pain Catastrophizing Scale for Children; PHODA = Photograph Series of Daily Activities; $\mathrm{SA}=$ subscore social activities; VAS = visual analogue scale. ${ }^{*}$ Values are mean \pm SD unless otherwise indicated. ${ }^{\text {a Normally }}$ distributed data. Values in parentheses in the test statistics column are $95 \%$ confidence interval. ${ }^{b}$ Not normally distributed data. Values are median (interquartile range). ${ }^{c}$ Only measured in the subgroup who received treatment (HM, $\mathrm{n}=14 ; \mathrm{NHM}, \mathrm{n}=31)$.

Before analyzing the association between PHODA-balance and disability, the Pearson correlation between FOPQ-C and PHODA-balance was calculated ( $r=0.36, p=0.01)$. Then, an identical procedure was performed using the PHODA-balance in a subgroup of adolescents who received multidisciplinary treatment instead of the FOPQ- $C$ as the fearrelated variable in the model. In the first step of the regression model, VAS, age, and sex were introduced, but no significant association with disability was found. The PHODA- 
balance and the group variable of hypermobility/no hypermobility were added in the second step $\left(R^{2}=0.44\right.$, adjusted $\left.R^{2}=0.36, p<0.001\right)$. Results showed that only the PHODAbalance was found to be associated with disability $(\beta=0.64, p<0.001)$, indicating that higher perceived harmfulness of balance-related activities was associated with more disabilities. In the third step, the interaction term (PHODA-balance $x$ hypermobility/nohypermobility) was added to the model, but no significant association with disability was found.

Table 3.3 Regression analysis with disability as dependent variable.*

\begin{tabular}{|c|c|c|c|c|c|}
\hline Independent variable & $\mathrm{B}$ & $\beta^{\mathrm{a}}$ & $\mathrm{R}^{2}$ & Adjusted $\mathrm{R}^{2}$ & F change \\
\hline Block 1 & & & 0.02 & -0.01 & 0.64 \\
\hline Sex & 0.05 & $0.00(-6.27,6,36)$ & & & \\
\hline Age & 0.20 & $0.05(-0.62,1.01)$ & & & \\
\hline VAS & 0.07 & $0.13(-0.04,0.18)$ & & & \\
\hline Block 2 & & & 0.32 & 0.29 & $21.39^{b}$ \\
\hline FOPQ-C & 0.37 & $0.56(0.26,0.48)^{b}$ & & & \\
\hline Group (HM/NHM) & -1.21 & $-0.06(-4.67,2.26)$ & & & \\
\hline Block 3 & & & 0.33 & 0.29 & 0.64 \\
\hline FOPQ-C $\times$ HM/NHM & 0.09 & $0.20(-0.13,0.32)$ & & & \\
\hline
\end{tabular}

Abbreviations: $\mathrm{FOPQ}-\mathrm{C}=$ Fear of Pain Questionnaire-Child report; $\mathrm{HM}=$ hypermobility; $\mathrm{NHM}=$ no hypermobility; VAS = visual analogue scale. ${ }^{*}$ Represented by the Functional Disability Inventory. ${ }^{\text {a }}$ Values in parentheses are $95 \%$ confidence interval. ${ }^{b} p<0.05$.

\section{Discussion}

The results indicate that hypermobile adolescents with CMP do not have more painrelated fear compared to non-hypermobile adolescents with $\mathrm{CMP}$, and that pain-related fear and being hypermobile do not reinforce each other in explaining the perceived level of disability. Further, the perceived harmfulness of balance-related activities was not more strongly associated with disability in hypermobile adolescents with CMP.

It was hypothesized that pain-related fear would be more prominent in hypermobile adolescents with CMP, possibly resulting in avoidance behaviour leading to impaired daily functioning. This was hypothesized to result in a stronger association between pain-related fear and disability in hypermobile as compared to non-hypermobile adolescents with CMP. However, results of the current study did not support these hypotheses. A possible explanation may be that the long-term experience of pain complaints in the included population resulted in deterioration of the daily functioning of not only the hypermobile but also the non-hypermobile adolescents with CMP. Being hypermobile probably makes you more vulnerable to developing chronic pain, but once 
you have chronic pain, the level of fear, as reported in the current study, is associated to disability.

The current study also explored whether the perceived harmfulness of balance-related activities had a stronger association with disability in hypermobile compared to nonhypermobile adolescents with CMP. The results did not confirm this supposition. Earlier studies reported a positive association between perceived harmfulness and the level of disability. ${ }^{49,52}$ This association was also found between perceived harmfulness of balance-related activities and disability in the current study, but this association was not stronger in hypermobile compared to non-hypermobile adolescents with CMP. However, the current findings are based on a limited sample of 45 adolescents (14 hypermobile and 31 non-hypermobile), so the results should be considered with caution. Nevertheless, earlier studies reported decreased muscle strength ${ }^{3}$, impaired proprioception $^{31}$, and reduced balance ${ }^{33}$ in hypermobile compared to non-hypermobile children and adolescents with CMP. Furthermore, adolescents with GJH prefer more stable activities (e.g., walking and cycling) and tend to avoid dynamic activities. ${ }^{40}$ Therefore, it might be of interest to evaluate the perceived harmfulness of balancerelated activities in adolescents with hypermobility in future studies.

As mentioned earlier, one limitation of the current study is the small number of adolescents who completed the PHODA-youth, resulting in insufficient power of the analysis that included the PHODA-balance. Furthermore, the selection of balance-related items (PHODA-balance) used in the current study, though made by experts, has not been validated. However, use of this subscale was necessary in this study to analyze the specific perceived harmfulness of balance-related activities. Further research is needed to confirm whether this selection of PHODA items offers a valid representation of balance-related activities. Another limitation is the cross-sectional design of the study, which meant that no causal relationships could be confirmed. However, if significant associations were not present in cross-sectional data, it is unlikely that they would be present in a longitudinal cohort.

This study confirms earlier findings that pain-related fear is related to disability. ${ }^{23,24}$ It is therefore important that, in clinical practice, treatment of adolescents with CMP focus on a biopsychosocial approach. ${ }^{4}$ This has also been confirmed in the last few decades by several biopsychosocial rehabilitation programmes for adults that focused on psychosocial influencing factors, and especially the influence of pain-related fear. ${ }^{16,17}$ 


\section{Conclusion}

The association of pain-related fear with the perceived level of disability is not more pronounced in hypermobile compared to non-hypermobile adolescents with CMP. Perceived harmfulness of balance-related activities was not more strongly associated with disability in hypermobile adolescents with CMP. 


\section{References}

1. Perquin CW, Hazebroek-Kampschreur AA, Hunfeld JA, Bohnen AM, van Suijlekom-Smit LW, Passchier J, et al. Pain in children and adolescents: a common experience. Pain. 2000;87(1):51-8.

2. Perquin CW, Hazebroek-Kampschreur AA, Hunfeld JA, van Suijlekom-Smit LW, Passchier J, van der Wouden JC. Chronic pain among children and adolescents: physician consultation and medication use. Clin J Pain. 2000;16(3):229-35.

3. El-Metwally A, Salminen JJ, Auvinen A, Kautiainen H, Mikkelsson M. Prognosis of non-specific musculoskeletal pain in preadolescents: a prospective 4-year follow-up study till adolescence. Pain. 2004;110(3):550-9.

4. O'Sullivan P, Beales D, Jensen L, Murray K, Myers T. Characteristics of chronic non-specific musculoskeletal pain in children and adolescents attending a rheumatology outpatients clinic: a crosssectional study. Pediatr Rheumatol Online J. 2011;9(1):3.

5. Mikkelsson M, El-Metwally A, Kautiainen H, Auvinen A, Macfarlane GJ, Salminen JJ. Onset, prognosis and risk factors for widespread pain in schoolchildren: a prospective 4-year follow-up study. Pain. 2008;138(3):681-7.

6. Konijnenberg AY, Uiterwaal CS, Kimpen JL, van der Hoeven J, Buitelaar JK, de Graeff-Meeder ER. Children with unexplained chronic pain: substantial impairment in everyday life. Arch Dis Child. 2005;90(7):680-6.

7. Merlijn VP, Hunfeld JA, van der Wouden JC, Hazebroek-Kampschreur AA, Passchier J, Koes BW. Factors related to the quality of life in adolescents with chronic pain. Clin J Pain. 2006;22(3):306-15.

8. Huguet A, Miro J. The severity of chronic pediatric pain: an epidemiological study. J Pain. 2008;9(3):22636.

9. Hunfeld JA, Perquin CW, Duivenvoorden HJ, Hazebroek-Kampschreur AA, Passchier J, van Suijlekom-Smit LW, et al. Chronic pain and its impact on quality of life in adolescents and their families. J Pediatr Psychol. 2001;26(3):145-53.

10. Eccleston C, Crombez G, Scotford A, Clinch J, Connell H. Adolescent chronic pain: patterns and predictors of emotional distress in adolescents with chronic pain and their parents. Pain. 2004;108(3):221-9.

11. Kashikar-Zuck S, Goldschneider KR, Powers SW, Vaught MH, Hershey AD. Depression and functional disability in chronic pediatric pain. Clin J Pain. 2001;17(4):341-9.

12. Gauntlett-Gilbert J, Eccleston C. Disability in adolescents with chronic pain: Patterns and predictors across different domains of functioning. Pain. 2007;131(1-2):132-41.

13. Stommen NC, Verbunt JA, Gorter SL, Goossens ME. Physical activity and disability among adolescents and young adults with non-specific musculoskeletal pain. Disabil Rehabil. 2012;34(17):1438-43.

14. Kashikar-Zuck S. Treatment of children with unexplained chronic pain. Lancet. 2006;367(9508):380-2.

15. McGhee JL, Burks FN, Sheckels JL, Jarvis JN. Identifying children with chronic arthritis based on chief complaints: absence of predictive value for musculoskeletal pain as an indicator of rheumatic disease in children. Pediatrics. 2002;110(2 Pt 1):354-9.

16. Vlaeyen JW, Linton SJ. Fear-avoidance and its consequences in chronic musculoskeletal pain: a state of the art. Pain. 2000;85(3):317-32.

17. Leeuw M, Goossens ME, Linton SJ, Crombez G, Boersma K, Vlaeyen JW. The fear-avoidance model of musculoskeletal pain: current state of scientific evidence. Journal of Behavioural Medicine. 2007;30(1):77-94.

18. Verbunt JA, Seelen HA, Vlaeyen JW, Bousema EJ, van der Heijden GJ, Heuts PH, et al. Pain-related factors contributing to muscle inhibition in patients with chronic low back pain: an experimental investigation based on superimposed electrical stimulation. Clin J Pain. 2005;21(3):232-40.

19. Verbunt JA, Seelen HA, Vlaeyen JW, van der Heijden GJ, Knottnerus JA. Fear of injury and physical deconditioning in patients with chronic low back pain. Arch Phys Med Rehabil. 2003;84(8):1227-32.

20. Verbunt JA, Smeets RJ, Wittink HM. Cause or effect? Deconditioning and chronic low back pain. Pain. 2010;149(3):428-30.

21. Verbunt JA, Seelen HA, Vlaeyen JW, van de Heijden GJ, Heuts PH, Pons K, et al. Disuse and deconditioning in chronic low back pain: concepts and hypotheses on contributing mechanisms. Eur J Pain. 2003;7(1):921. 
22. Verbunt JA, Westerterp KR, van der Heijden GJ, Seelen HA, Vlaeyen JW, Knottnerus JA. Physical activity in daily life in patients with chronic low back pain. Arch Phys Med Rehabil. 2001;82(6):726-30.

23. Simons LE, Kaczynski KJ. The Fear Avoidance model of chronic pain: examination for pediatric application. The Journal of Pain. 2012;13(9):827-35.

24. Asmundson GJ, Noel M, Petter M, Parkerson HA. Pediatric fear-avoidance model of chronic pain: foundation, application and future directions. Pain Res Manag. 2012;17(6):397-405.

25. Tobias JH, Deere K, Palmer S, Clark EM, Clinch J. Joint hypermobility is a risk factor for musculoskeletal pain during adolescence: findings of a prospective cohort study. Arthritis Rheum. 2013;65(4):1107-15.

26. Sohrbeck-Nohr O, Kristensen JH, Boyle E, Remvig L, Juul-Kristensen B. Generalized joint hypermobility in childhood is a possible risk for the development of joint pain in adolescence: a cohort study. BMC Pediatr. 2014;14:302.

27. Gedalia A, Press J, Klein M, Buskila D. Joint hypermobility and fibromyalgia in schoolchildren. Ann Rheum Dis. 1993;52(7):494-6.

28. Ting TV, Hashkes PJ, Schikler K, Desai AM, Spalding S, Kashikar-Zuck S. The role of benign joint hypermobility in the pain experience in Juvenile Fibromyalgia: an observational study. Pediatr Rheumatol Online J. 2012;10(1):16.

29. Siegel DM, Janeway D, Baum J. Fibromyalgia syndrome in children and adolescents: clinical features at presentation and status at follow-up. Pediatrics. 1998;101(3 Pt 1):377-82.

30. Clinch J, Deere K, Sayers A, Palmer S, Riddoch C, Tobias JH, et al. Epidemiology of generalized joint laxity (hypermobility) in fourteen-year-old children from the UK: a population-based evaluation. Arthritis Rheum. 2011;63(9):2819-27.

31. Fatoye F, Palmer S, Macmillan F, Rowe $P$, van der Linden M. Proprioception and muscle torque deficits in children with hypermobility syndrome. Rheumatology (Oxford). 2009;48(2):152-7.

32. Zekry OA, Ahmed MA, Elwahid HAEA. The impact of fatigue on health related quality of life in adolescents with benign joint hypermobility syndrome. Egypt Rheumatol. 2013;35:77-85.

33. Schubert-Hjalmarsson E, Ohman A, Kyllerman M, Beckung E. Pain, balance, activity, and participation in children with hypermobility syndrome. Pediatric Physical Therapy. 2012;24(4):339-44.

34. Adib N, Davies K, Grahame R, Woo P, Murray KJ. Joint hypermobility syndrome in childhood. A not so benign multisystem disorder? Rheumatology (Oxford). 2005;44(6):744-50.

35. Bulbena A, Gago J, Pailhez G, Sperry L, Fullana MA, Vilarroya O. Joint hypermobility syndrome is a risk factor trait for anxiety disorders: a 15-year follow-up cohort study. Gen Hosp Psychiatry. 2011;33(4):36370.

36. Garcia-Campayo J, Asso E, Alda M. Joint hypermobility and anxiety: the state of the art. Curr Psychiatry Rep. 2011;13(1):18-25.

37. Garcia Campayo J, Asso E, Alda M, Andres EM, Sobradiel N. Association between joint hypermobility syndrome and panic disorder: a case-control study. Psychosomatics. 2010;51(1):55-61.

38. Smith TO, Easton V, Bacon H, Jerman E, Armon K, Poland F, et al. The relationship between benign joint hypermobility syndrome and psychological distress: a systematic review and meta-analysis. Rheumatology (Oxford). 2014;53(1):114-22.

39. Cederlof M, Larsson H, Lichtenstein P, Almqvist C, Serlachius E, Ludvigsson JF. Nationwide populationbased cohort study of psychiatric disorders in individuals with Ehlers-Danlos syndrome or hypermobility syndrome and their siblings. BMC Psychiatry. 2016;16:207.

40. Scheper M, de Vries J, Beelen A, de Vos R, Nollet F, Engelbert R. Generalized joint hypermobility, muscle strength and physical function in healthy adolescents and young adults. Curr Rheumatol Rev. 2014;10(2):117-25.

41. Bashe P, Greydanus DE. The American Academy of Pediatrics: Caring for your teenager. New York: Bantam Books; 2003.

42. Smits-Engelsman B, Klerks M, Kirby A. Beighton score: a valid measure for generalized hypermobility in children. The Journal of Pediatrics. 2011;158(1):119-23.

43. Juul-Kristensen B, Kristensen JH, Frausing B, Jensen DV, Rogind H, Remvig L. Motor competence and physical activity in 8-year-old school children with generalized joint hypermobility. Pediatrics. 2009;124(5):1380-7. 
44. Stinson JN, Kavanagh T, Yamada J, Gill N, Stevens B. Systematic review of the psychometric properties, interpretability and feasibility of self-report pain intensity measures for use in clinical trials in children and adolescents. Pain. 2006;125(1-2):143-57.

45. McGrath PJ, Walco GA, Turk DC, Dworkin RH, Brown MT, Davidson K, et al. Core outcome domains and measures for pediatric acute and chronic/recurrent pain clinical trials: PedIMMPACT recommendations. J Pain. 2008;9(9):771-83.

46. Walker LS, Greene JW. The functional disability inventory: measuring a neglected dimension of child health status. J Pediatr Psychol. 1991;16(1):39-58.

47. Claar RL, Walker LS. Functional assessment of pediatric pain patients: psychometric properties of the functional disability inventory. Pain. 2006;121(1-2):77-84.

48. Simons LE, Sieberg CB, Carpino E, Logan D, Berde C. The Fear of Pain Questionnaire (FOPQ): assessment of pain-related fear among children and adolescents with chronic pain. J Pain. 2011;12(6):677-86.

49. Verbunt JA, Nijhuis A, Vikstrom M, Stevens A, Haga N, de Jong J, et al. The psychometric characteristics of an assessment instrument for perceived harmfulness in adolescents with musculoskeletal pain (PHODAyouth). European Journal of Pain. 2015;19(5):695-705.

50. Crombez G, Bijttebier P, Eccleston C, Mascagni T, Mertens G, Goubert L, et al. The child version of the pain catastrophizing scale (PCS-C): a preliminary validation. Pain. 2003;104(3):639-46.

51. Myers RH. Classical and Modern Regression with Applications. Belmont, California.: Duxbury Press; 1990.

52. Simons LE, Pielech M, McAvoy S, Conroy C, Hogan M, Verbunt JA, et al. Photographs of Daily ActivitiesYouth English: validating a targeted assessment of worry and anticipated pain. Pain. 2017;158(5):912-21. 


\section{Chapter 4}

Generalized joint hypermobility and perceived harmfulness in healthy adolescents; impact on muscle strength, motor performance, and physical activity level
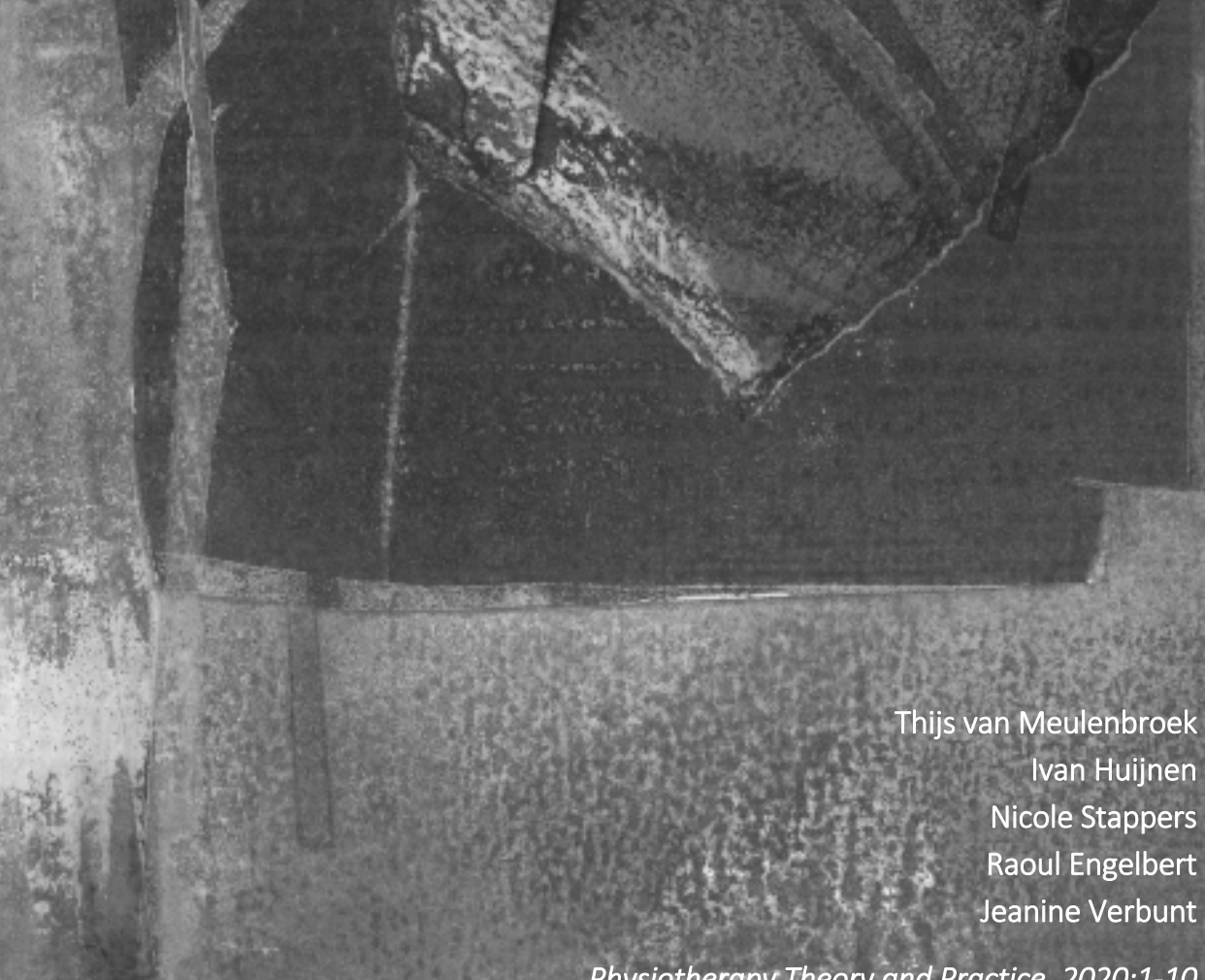

Physiotherapy Theory and Practice. 2020:1-10 


\section{Abstract}

\section{Objective}

The first aim of this study was to determine whether adolescents with asymptomatic generalized joint hypermobility (GJH) have a lower level of physical functioning (physical activity level, muscle strength, and motor performance) compared to non-hypermobile controls. Secondly, to evaluate whether the negative impact of perceived harmfulness on physical functioning was more pronounced in adolescents with asymptomatic GJH.

\section{Methods}

Cross-sectional study. Sixty-two healthy adolescents (mean age 16.8, range 12-21) participated. Hypermobility (Beighton score), perceived harmfulness (PHODA-youth), muscle strength (dynamometry), motor performance (single leg hop for distance), and physical activity level (PAL) (accelerometry) were measured. Hierarchical regression analyses were used to study differences in physical functioning and perceived harmfulness between asymptomatic GJH and non-hypermobile controls.

\section{Results}

Asymptomatic GJH was associated with increased knee extensor muscle strength (peak torque/body weight; PT/BW), controlled for age and gender (dominant leg; $\beta=0.29$, $p=0.02$ ). No other associations between asymptomatic GJH and muscle strength, motor performance, and PAL were found. Perceived harmfulness was not more pronounced in adolescents with asymptomatic GJH.

\section{Conclusions}

Adolescents with asymptomatic GJH had increased knee extensor muscle strength compared to non-hypermobile controls. No other differences in the level of physical functioning was found and the negative impact of perceived harmfulness was not more pronounced in adolescents with asymptomatic GJH. 


\section{Introduction}

Generalized joint hypermobility (GJH) is defined as an increased range of motion in most of the individual's joints. ${ }^{1}$ The prevalence of GJH in different child and adolescent populations varies from 2-55\%, depending on ethnicity, gender, age, and the tests and criteria applied. ${ }^{2}$ For many individuals with GJH, flexible joints deliver a potential benefit in the performance of sporting activities, such as dancing or gymnastics. ${ }^{3}$ However, for others, GJH can be disabling since it is related to symptoms such as chronic pain, joint instability, disturbed proprioception, and soft tissue injuries, and is referred to as generalized hypermobility spectrum disorder (G-HSD). ${ }^{4}$ G-HSD shows overlap with the hypermobile type of the Ehlers-Danlos syndrome (hEDS). ${ }^{5}$ Both symptomatic conditions lack a specific genetic profile and it is referred to as G-HSD/hEDS.

In individuals with G-HSD/hEDS, a reduction in level of physical functioning ${ }^{6,7}$, decreased muscle strength ${ }^{8,9}$, impaired proprioception ${ }^{9,10}$, reduced balance ${ }^{11}$, and decreased physical activity levels (PAL) $)^{12}$ are commonly reported. Earlier studies also indicated that asymptomatic GJH alone was associated with decreased daily activities, such as walking distance and jumping capacity ${ }^{13}$, and decreased muscle strength. ${ }^{14,15}$ In addition, outcomes of a physical activity questionnaire showed that adolescents and young adults with asymptomatic GJH participated less during sports and outdoor activities and preferred more stable activities, such as walking and cycling compared to nonhypermobile controls. ${ }^{15}$ However, other studies could not confirm the reduced muscle strength $^{16,17}$ and altered physical activity level measured objectively with an accelerometer ${ }^{18}$ in adolescents and adults with asymptomatic GJH vs non-hypermobile controls. A possible explanation might be that the included population in the study of Jensen et al. ${ }^{16}$ and Mebes et al. ${ }^{17}$ was older than the included population in the studies that did find decreased muscle strength in asymptomatic GJH.

Thus, it remains unclear whether a lower level of physical functioning in G-HSD/hEDS is caused by GJH itself or whether it is due to pain or the anticipation of pain. It could be hypothesized that, due to $\mathrm{GJH}$, an individual may learn to avoid or to be more careful during complex activities requiring more joint control, in order to prevent the development of musculoskeletal complaints or injuries. This behaviour seems in line with avoidance behaviour described in the fear-avoidance model to explain the disabling role of pain-related fear in chronic pain. ${ }^{19,20}$ However, it is unclear whether individuals with asymptomatic GJH also perceive complex activities as potentially more harmful compared to individuals without GJH.

Therefore, the aim of this study was to determine whether adolescents with asymptomatic GJH have lower physical functioning levels, by assessing muscle strength, motor performance, and PAL compared to non-hypermobile adolescents. In addition, 
the second aim was to evaluate whether the negative impact of perceived harmfulness on muscle strength, motor performance, and PAL was more pronounced in adolescents with asymptomatic GJH compared to non-hypermobile adolescents.

\section{Methods}

\section{Participants}

In this cross-sectional study, sixty-two healthy adolescents (mean age 16.8, SD 2.3, range 12-21) were recruited in the Southern area of the Netherlands. The adolescents had good understanding of the Dutch language. Exclusion criteria were chronic musculoskeletal pain and specific medical conditions/disorders influencing physical functioning, such as acute or recurrent musculoskeletal pain. The study protocol was approved by the Medical Ethics Committee of the Academic Hospital Maastricht/Maastricht University, the Netherlands (METC number: 15-4-052).

\section{Procedure}

Adolescents were included in three different ways. First, adolescents were verbally informed about the purpose of the study on a local high school. Second, adolescents responded on a pamphlet that was presented at two institutes for higher education (Zuyd University of Applied Science and Maastricht University). Third, adolescents were asked from the personal network of the research group. Adolescents, that indicated to be interested in participation received an information letter and after a week, a researcher (T.M. / N.S. / T.H.) contacted them by telephone or by mail to ask whether they were willing to participate in the study. All adolescents who participated provided written informed consent. Adolescents younger than 18 years needed written approval from their parents and/or caregivers.

After written approval all outcome measures were collected by a physical therapist during a 1 hour session. First, sociodemographic variables (age, gender, ethnicity of the father and mother, education level, and if applicable having a (part time) job), pain intensity, and perceived harmfulness were collected. Further height and weight were measured in a standardized method without heavy clothing and shoes. BMI was calculated as weight in kilograms divided by the square of height in meters. Then, physical functioning (i.e. isokinetich muscle strength, isokinetic muscle strength endurance, and motor performance) was assessed and GJH was measured using the Beighton score (BS). At last, adolescents were instructed about the use of the 
accelerometer, which measured PAL. The accelerometer had to be worn during 7 consecutive days.

\section{Measurements}

\section{Hypermobility}

The presence of hypermobility was assessed using the BS, with a standardized protocol. $^{21}$ The BS consists of 9 functional tests (hyperextension of the knees, elbows and little fingers, thumb apposition to the forearm, and trunk flexion) and scored dichotomously with a maximum score of 9 points. In a recently published consensus statement a cut-off point of 5 out of 9 for adults up to the age of 50 and a cut-off point of 6 out of 9 for pre-pubertal children and adolescents was recommended., ${ }^{5,22}$ Based on these findings, the adolescents were assigned to one of two groups: a group with asymptomatic GJH (GJH: BS $\geq 6$ for $<18$ years and $\mathrm{BS} \geq 5$ for $\geq 18$ years) or a group without asymptomatic GJH (non-GJH: BS $<6$ for $<18$ years and $\mathrm{BS}<5$ for $\geq 18$ years). The $\mathrm{BS}$ is a valid instrument to evaluate $\mathrm{GJH}$ in elementary schoolchildren. ${ }^{21}$

\section{Pain intensity}

Pain intensity was measured with three 100-mm visual analogue scales (VAS): (1) current pain, (2) the worst/most severe pain experienced in the last week, and (3) the least pain experienced in the last week. The VAS is a reliable and valid measure for pain intensity in children older than 8 years. ${ }^{23}$ The mean of these 3 VAS scores was calculated and used to express pain intensity in the analyses.

\section{Perceived harmfulness (PHODA-youth)}

The Photograph Series of Daily Activities for youth (PHODA-youth), which has been found to be valid and reliable in adolescents with chronic musculoskeletal pain ${ }^{24}$, was used to measure perceived harmfulness. The PHODA-youth contains 51 age-specific activities and social situations and consists out of 3 categories: (1) activities of daily living and household (PHODA-ADL; 13 items), (2) intensive physical activities (PHODA-PA; 27 items), and (3) social activities (PHODA-SA; 11 items). Adolescents rate the photographs from 0 (not harmful at all) to 10 (extremely harmful), where higher scores indicated higher levels of perceived harmfulness.

\section{Physical functioning}

To represent the level of physical functioning; isokinetic muscle strength and endurance, motor performance, and physical activity level were assessed. 


\section{Muscle strength}

The Biodex System 3 Pro dynamometer (Biodex Medical Systems, Shirley, NY, USA), which is a reliable and valid isokinetic dynamometer ${ }^{25}$, was used to test isokinetic knee extensor and flexor strength in both legs. The adolescent's leg dominance was recorded by asking which leg was preferred for kicking a ball. The set-up of Rombaut et al. ${ }^{26}$ was used, where the adolescent was positioned in an upright sitting position and the tested leg was stabilized with a fixation strap. The lever arm was attached to the adolescent's lower leg by a padded cuff $2 \mathrm{~cm}$ proximal to the medial malleolus, and the axis of movement of the dynamometer was in line with the axis of movement of the knee extension/flexion. After one practice repetition to become familiar with the movement and velocity, five repetitions of maximal voluntary concentric knee extension and flexion were performed at the angular velocity of $60^{\circ} /$ second. Peak torque (PT, Nm) was assessed and is the highest muscular force output similar to one repetition maximum effort in isotonic muscle work and represents the muscle's maximum strength capability. PT/body weight is the PT normalized for body weight, used to standardize and compare scores and was used for further analyses.

\section{Muscle strength endurance}

The same setup as described above was used for measuring isokinetic muscle strength endurance in both legs. The adolescent had to perform 30 repetitions of maximal concentric knee extension and flexion with an angular velocity of $240 \%$ second. The test was performed 60 seconds after the isokinetic test of $60^{\circ} /$ second. Total work $(\mathrm{J})$ is the work produced throughout the test and represents the muscle's capability.

\section{Motor performance}

The single leg hop for distance (SLHD) is a dynamic motor performance test ${ }^{27}$ measuring joint stability and coordination ${ }^{28}$, which might be decreased in asymptomatic GJH. The SLHD has shown high intra-subject reliability. ${ }^{29}$ The method of Junge et al. ${ }^{27}$ was used, where the adolescent stood on the test leg and then hopped as far as possible allowing arm swing assistance and landed on the same leg. The adolescent was instructed to control their landing and hold the landing foot in place until the distance was registered. After one practice hop, three valid hops with both legs were performed with a resting period of 30 seconds in between. The greatest distance measured in centimetres from the toe at the push off to the heel at the place the adolescents landed, for the dominant as well as the non-dominant leg was used for the analyses. 


\section{Physical activity level}

The level of physical activity (PAL) was measured using a tri-axial accelerometer ( $\mathrm{AX} 3$; Axivity, Newcastle, UK). The accelerometer was attached to the hip at the dominant side of the body with the USB port at the downside, medial to the spina iliaca anterior superior (SIAS), using plastic film (Tegaderm Film, $10 \times 12 \mathrm{~cm}$ ) and had to be removed during the performance of activities that could damage the monitor, such as contact sports and swimming. Bathing and showering was allowed with the accelerometer. Each adolescent received a diary to collect information on data interruption and was asked to register non-wearing time and the reason for doing this. ${ }^{30}$ Furthermore, wake up time, sleeping, and school hours were noted. Nighttime and periods of non-wearing activities were excluded from the analyses. To be included as a valid score, a minimum of three weekdays with at least 10 hours of recording and one weekend day with at least 8 hours of recording had to be available during the 7-day monitoring period. ${ }^{31,32}$ Data processing was performed using Matlab (The Math Works Inc., Natick, MA, USA). To obtain activity counts, an algorithm was designed based on the method, which was used for the Actiwatch 7 (Philips, Eindhoven, the Netherlands). Using this algorithm, the acceleration signals were converted into one resultant acceleration signal. This signal was rectified and filtered using a high-pass filter at $3 \mathrm{~Hz}$ and a low pass filter at $10 \mathrm{~Hz}$. The highest recorded sample per second was selected in the filtered and rectified data and summed per minute. This counts/minute signal was used for all further calculations. Daily uptime was defined as the period between getting up and going to sleep and was presented in minutes.

PAL was expressed in three quantities: (1) total activity during uptime, calculated as the total sum of counts during uptime. ${ }^{33}$ Data imputation was used to compensate for nonwearing time during contact sports. Values during non-wearing time were replaced by 2 times the mean score per minute of the previous day ${ }^{33}$; (2) mean activity level during 24 hours, expressed as the mean number of counts per minute per day. In this second method, sleeping time is included in the total score; and (3) peak activity level, determined as the highest number of counts achieved in a single minute per daytime wake period. $^{34}$ In addition, total activity during uptime and peak activity level were calculated separately for the weekend days, weekdays, school hours, and non-school hours.

\section{Statistical analysis}

Data analysis was performed using IBM Statistics for Windows, v23.0 (IBM Corp, Armonk, NY, USA). Normality of the variables was checked by eye balling using histograms and the Kolmogorov-Smirnov goodness of-fit-test. Descriptive data of the 
anthropometrics and sociodemographic measures, perceived harmfulness, and pain intensity of the GJH group and the non-GJH group are presented as mean \pm standard deviation (SD) in the case of normality of the variable. In the case of a non-normal distribution the median and interquartile range (IQR) are presented. Group differences between non-GJH and GJH were tested using an independent sample $t$ test, MannWhitney $U$ test or Kruskall-Wallis test for numerical variables and a chi-square test for categorical variables.

Hierarchical regression analyses were used to study the hypothesis that adolescents with asymptomatic GJH have a decreased level of physical functioning (represented by muscle strength, muscle strength endurance, motor performance, and PAL) compared to non-hypermobile adolescents and in addition, whether the negative impact of perceived harmfulness is more pronounced in adolescents with asymptomatic GJH. Analyses were performed per representative of physical functioning in three steps: (1) representative of physical functioning (i.e. muscle strength) was introduced as the dependent variable and age, gender (female=0; male=1), and hypermobility (non-GJH=0; GJH=1) were introduced as independent variables; (2) PHODA-youth was added; and (3) interaction term (PHODA-youth $\times$ GJH/non-GJH) was introduced. In case of a significant interaction, additional regression analyses were performed for both groups separately. This procedure was repeated with the other representative of physical functioning (i.e. muscle strength endurance, motor performance, and PAL [total activity during uptime]) as independent variables. For all independent variables, the association with the outcome was presented by the standardized regression coefficient $(\beta)$ and $p$-value. As muscle strength endurance (extension dominant leg and extension non-dominant leg), the PHODA-youth, and age did not meet the assumption of normality, these variables were log transformed prior to analyses. After transformation, skewness and kurtosis values of the scales were in the acceptable range of -1 and +1 . For all regression analyses, a collinearity check was performed. Collinearity was considered a problem when the variance inflation factor (VIF) was above $10{ }^{35}$ To compare the different PAL conditions between the GJH and non-GJH group, independent $t$ tests or Mann-Whitney $U$ tests were used. $P$-values less than 0.05 were considered statistically significant.

\section{Results}

\section{Descriptive analyses}

Sixty-two adolescents (47 females, 15 males) participated in the study. Table 4.1 presents the characteristics per group (GJH/non-GJH). Eleven adolescents (18\%) fulfilled the criteria for asymptomatic GJH, only 1 male had GJH. There was a significantly higher 
Beighton $(p<0.01)$ and higher age $(p=0.02)$ in the GJH group compared to the non-GJH group. In addition, significantly more adolescents in the GJH group participated in tertiary education (vocational education or university of applied sciences or university) compared to the non-GJH group ( $p=0.04)$. No other significant differences between the GJH and the non-GJH group with respect to gender, weight, height, BMI, ethnicity, part time job, and pain intensity were found.

Table 4.1 Characteristics of the study sample of adolescents with or without asymptomatic GJH.

\begin{tabular}{|c|c|c|c|}
\hline & $\mathrm{GJH}(\mathrm{n}=11)$ & Non-GJH (n=51) & Test statistics \\
\hline Females/males, $n$ & $10 / 1$ & $37 / 14$ & $p=0.27$ \\
\hline Beighton, $n^{a}$ & $6.0(5.0-7.0)$ & $2.0(2.0-3.0)$ & $U=8, z=-5.23, p<0.01$ \\
\hline Age, $y^{a}$ & $18.0(16.0-20.0)$ & $16.0(15.0-18.0)$ & $U=158, z=-2.28, p=0.02$ \\
\hline Weight, $\mathrm{kg}^{\mathrm{a}}$ & $63.1(52.0-73.7)$ & $59.0(53.1-69.7)$ & $U=259, z=-0.40, p=0.69$ \\
\hline Height, $\mathrm{cm}^{\mathrm{b}}$ & $169.7 \pm 10.1$ & $170.0 \pm 9.0$ & $t_{60}=0.09(0.28,3.06)$ \\
\hline $\mathrm{BMI}, \mathrm{kg} / \mathrm{m}^{2 \mathrm{a}}$ & $22.4(18.5-24.2)$ & $21.5(18.9-23.0)$ & $U=259, z=-0.40, p=0.69$ \\
\hline Ethnicity father, n (\%) & & & $\mathrm{H}_{1}=0.01, p=0.92$ \\
\hline Caucasian & $10(90.9)$ & $48(94.2)$ & \\
\hline Arabic & $1(9.1)$ & $2(3.9)$ & \\
\hline Latin American & $0(0.0)$ & $1(2.0)$ & \\
\hline Ethnicity mother, n (\%) & & & $\mathrm{H}_{1}=0.00, p=0.97$ \\
\hline Caucasian & $10(90.9)$ & $48(94.2)$ & \\
\hline Arabic & $1(9.1)$ & $2(3.9)$ & \\
\hline Latin American & $0(0.0)$ & $1(2.0)$ & \\
\hline Education level, n (\%) & & & $\mathrm{H}_{1}=4.09, p=0.04$ \\
\hline Primary education & $0(0.0)$ & $1(2.0)$ & \\
\hline Secondary education & $3(27.3)$ & $30(58.8)$ & \\
\hline Tertiary education & $8(72.7)$ & $20(39.2)$ & \\
\hline Part time job (yes/no), n & $9 / 2$ & $33 / 18$ & $p=0.48$ \\
\hline Pain intensity ${ }^{a}$ & $0.5(0.0-0.9)$ & $0.3(0.0-1.1)$ & $U=276, z=-0.09, p=0.93$ \\
\hline
\end{tabular}

Abbreviations: GJH = group with asymptomatic hypermobility; Non-GJH = group without asymptomatic hypermobility; $\mathrm{BMI}=$ body mass index. ${ }^{a}$ Not normally distributed data are presented by median (IQR). ${ }^{\mathrm{b}}$ Normally distributed data are presented by mean \pm SD.

Table 4.2 presents the results of the level of perceived harmfulness on the PHODA-youth and all subscales. Results showed that the level of the perceived harmfulness was very low on all subscales related to the total score range. There were no significant differences regarding the level of perceived harmfulness between the GJH group and the non-GJH group (Table 4.2).

\section{Muscle strength}

Table 4.3 presents the results of muscle strength. The first step showed that having GJH ( $\beta=0.29, p=0.02$ ) and a higher age ( $\beta=0.26, p=0.04)$ were both associated with increased knee extensor muscle strength (PT/BW). The mean difference of the knee extensor muscle strength for respectively females and males in the GJH group was $45 \%$ and $46 \%$ 
higher in the dominant leg and $36 \%$ and $28 \%$ higher in the non-dominant leg compared to the females and males in the non-GJH group. In the second step, the PHODA-youth was introduced. No significant association between the PHODA-youth score and muscle strength (PT/BW) were found. After adding the interaction term (PHODA-youth $x$ GJH/non-GJH) in step 3, no significant association was found. There were no significant associations regarding the knee flexion muscle strength (PT/BW). The analyses with the non-dominant leg were not presented but results were comparable to the findings as presented of the dominant leg.

Table 4.2 Outcome of the PHODA-youth questionnaire.

\begin{tabular}{lccc}
\hline & $\mathrm{GJH}(\mathrm{n}=11)$ & Non-GJH $(\mathrm{n}=51)$ & Test statistics \\
\hline PHODA ADL (range; 0-130) & $0.0(0.0-1.0)$ & $0.0(0.0-2.0)$ & $\mathrm{U}=231, z=-1.02, p=0.31$ \\
PHODA PA (range; 0-270) & $2.5(1.0-11.5)$ & $5.5(1.0-24.0)$ & $\mathrm{U}=260, z=-0.38, p=0.70$ \\
PHODA SA (range; 0-110) & $0.0(0.0-2.0)$ & $1.0(0.0-7.0)$ & $\mathrm{U}=187, z=-1.81, p=0.07$ \\
PHODA total (range; 0-510) & $5.0(2.0-16.5)$ & $8.0(1.0-38.0)$ & $\mathrm{U}=239, z=-0.78, p=0.44$ \\
\hline
\end{tabular}

Abbreviations: GJH = group with asymptomatic hypermobility; Non-GJH = group without asymptomatic hypermobility; PHODA = Photograph Series of Daily Activities; PHODA ADL = subscore daily life activities; PHODA PA = subscore physical activities; PHODA SA = subscore social activities. All variables were not normally distributed and therefore presented by median (IQR).

\section{Muscle strength endurance}

For the flexor and extensor muscle strength endurance (total work), there were no differences between the GJH group and the non-GJH group (Table 4.3). Only a higher age was associated with a higher knee extensor $(\xi=0.27, p=0.04)$ and flexor $(\xi=0.32$, $p=0.02$ ) muscle strength endurance (total work). Analyses with the non-dominant leg were not presented but results were comparable to outcomes of the dominant leg as presented.

\section{Motor performance}

According to motor performance (SLHD), there were no significant differences between the GJH group and the non-GJH group (Table 4.3). Results in the first step showed that male adolescents had a significantly higher score on the SLHD $(\beta=0.45, p<0.01)$. In the second step, the PHODA-youth was associated with motor performance $(\beta=-0.33$, $p=0.01$ ), indicating that higher perceived harmfulness was associated with a lower score on the SLHD, but this finding was not different for those with and without hypermobility. The analysis with the non-dominant leg was not presented but again results were comparable with the results of the dominant leg. 
GJH and perceived harmfulness in healthy adolescents; impact on physical functioning

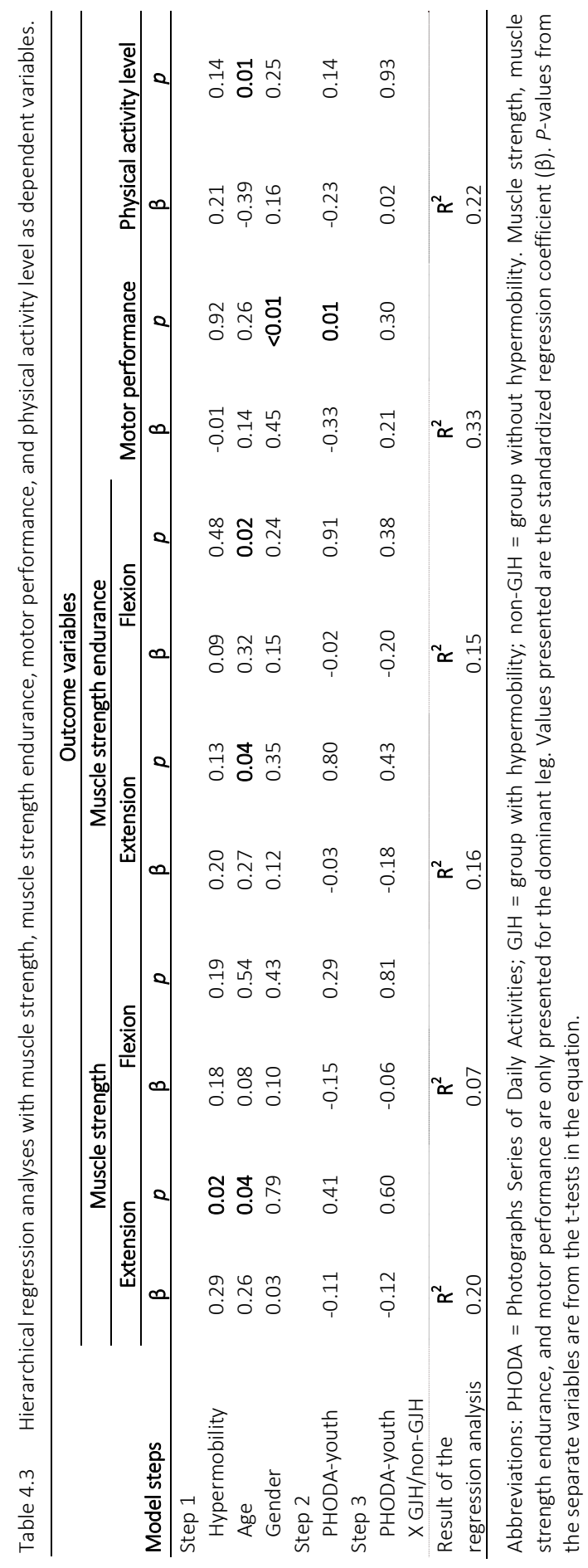




\section{PAL}

The accelerometer data of 49 adolescents $(\mathrm{GJH}, \mathrm{n}=8$; non-GJH, $n=41)$ were used for analysis of PAL. Data of thirteen of the 62 adolescents were excluded from the analysis due to invalid scores caused by dysfunction of the $\operatorname{AX3}(n=3)$ or due to not fulfilling the pre-determined criteria of the weekly registration for a valid accelerometer registrationperiod ( $n=10)$. During PAL measurements, 15 adolescents had no school obligations due to holidays. Therefore only $34(\mathrm{GJH}, \mathrm{n}=5$; non-GJH, $\mathrm{n}=29)$ participated in school. Figure 4.1 presents the overall total activity during uptime and total activity during uptime per week and weekend day, and school and non-school hours for the GJH and non-GJH group separately. There were no significant differences between the GJH and the non-GJH group for all PAL conditions. In addition, daily uptime was not significantly different between both groups.

In Table 4.3 the outcome of the regression model with PAL (total activity during uptime) showed that in the first step a younger age was associated with an increased level of PAL ( $\beta=-0.39, p<0.01$ ). There were no significant differences in PAL between the GJH group and the non-GJH group. The analyses with mean activity level during 24 hours and peak activity level instead of total activity during uptime were not presented but confirmed the findings of total activity during uptime.

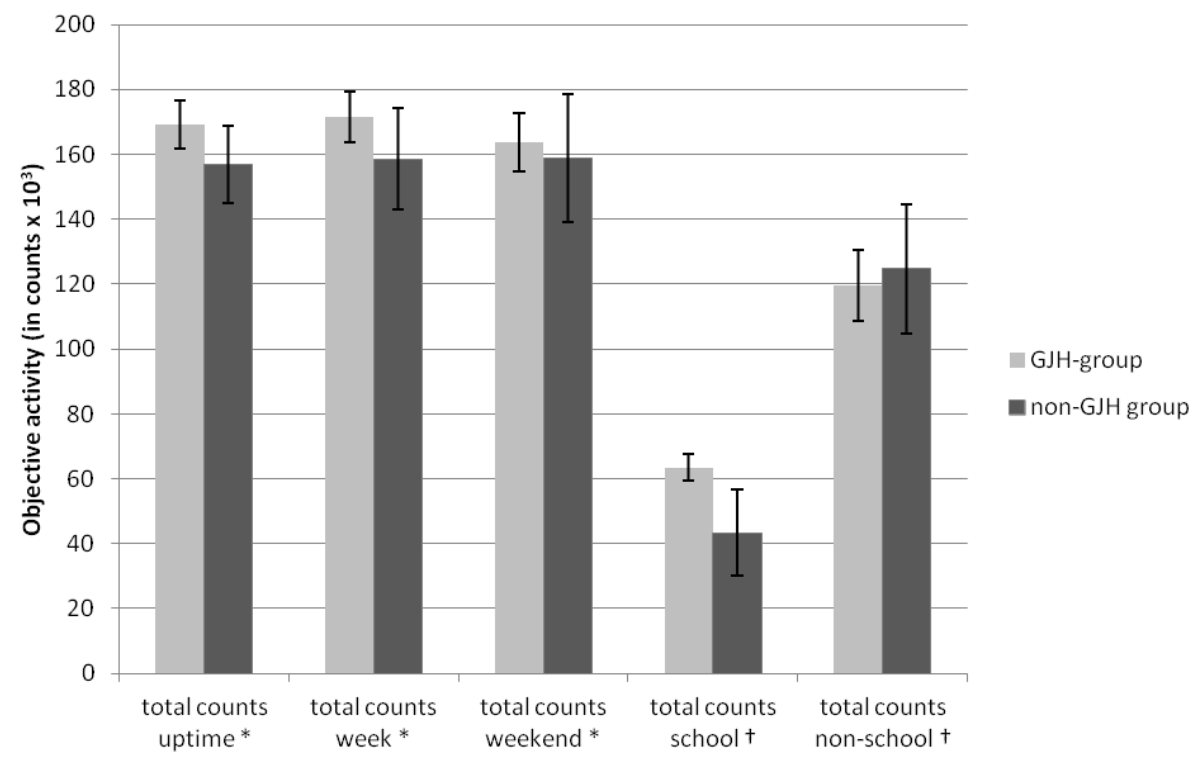

Figure 4.1 Mean differences in total activity during uptime between the GJH and non-GJH group.

The error bars represent the standard error of the mean. ${ }^{*} \mathrm{GJH}(n=8)$ and non-GJH $(n=41)$. $+\mathrm{GJH}$ $(n=5)$ and non-GJH $(n=29)$. 


\section{Discussion}

This study indicates that adolescents with asymptomatic GJH have no lower physical functioning levels, regarding muscle strength, motor performance, and PAL compared to non-hypermobile adolescents. Contrary to expectations, adolescents with asymptomatic GJH have increased muscle strength of the knee extensors of the dominant and nondominant leg compared to non-hypermobile adolescents. In addition, the negative impact of perceived harmfulness was not more pronounced in adolescents with asymptomatic GJH.

In contradiction to earlier studies that did find a lower level of physical functioning in adolescents and young adults with asymptomatic GJH compared to non-hypermobile controls $^{13-15}$, our results showed that adolescents with asymptomatic GJH have no decreased muscle strength, motor performance, and PAL compared to non-hypermobile adolescents. However, the findings of the current study were consistent with results of earlier studies in adolescents and adults with asymptomatic GJH compared to nonhypermobile controls. ${ }^{16-18,27}$ In addition, a recent study also showed that no differences in physical functioning, measured as lower extremity function and motor performance, occurred between adolescent athletes with asymptomatic GJH and non-hypermobile adolescent athletes. ${ }^{36}$ A possible explanation between the different outcomes of PAL could be that the current study and Clinch et al. ${ }^{18}$ used accelerometry as objective measurement, whereas Scheper el al. ${ }^{15}$ used a subjective measure. So, adolescents with GJH might perceive an altered PAL but objective measurements did not confirm these beliefs. Furthermore, our study, Jensen et al. ${ }^{16}$, Junge et al. ${ }^{27}$, and Mebes et al. ${ }^{17}$ found no differences in muscle strength of the lower extremity in contrast to Jindal, Narayn, Ganesan and MacDermid ${ }^{14}$ and Scheper et al. ${ }^{15}$ who also measured upper extremity. Therefore, it might be recommendable to measure lower and upper extremity muscle strength in future studies as well as subjective and objective measurements of PAL. The results of the current study even showed that muscle strength of the knee extensors was increased in adolescents with asymptomatic GJH compared to non-hypermobile adolescents, which was also found in a study of Mebes et al. ${ }^{17}$ with a female population (aged 27.2-28.1 years). Thus, having asymptomatic GJH appeared to be non-related to a decrease in physical functioning. However, more research according to the updated cutoff values of the recent published consensus statement ${ }^{5,22}$ is needed to confirm these findings and whether muscle strength is even increased in adolescents with asymptomatic GJH.

The objective physical measurements in the current study showed that, having asymptomatic GJH was not associated with decreased physical functioning. In patients with G-HSD/hEDS, decreased muscle strength ${ }^{8,9}$, reduced balance ${ }^{11}$, decreased PAL, and 
lower sport participation ${ }^{11,12}$ were found compared to pain-free non-hypermobile controls. These results seemed to imply that a lower level of physical functioning in patients with G-HSD/hEDS could not be explained by GJH alone. This is also confirmed in a recent longitudinal study in children with G-HSD/hEDS, in which multi-systemic dysfunction (e.g. skin, cardiovascular, and gastrointestinal involvement), high levels of pain and fatigue, and loss of postural control were found to be the most important constructs for functional impairment in terms of performance and capacity. ${ }^{37}$ However, given that the findings of the current study are based on a small number of adolescents with asymptomatic $\mathrm{GJH}$, this interpretation should be treated with caution.

Results of the current study also showed that perceived harmfulness was not associated with a decreased muscle strength nor PAL. However, perceived harmfulness was associated with a lower score on the motor performance task. Although hypothesized, this finding was not more pronounced in adolescents with asymptomatic GJH. One recent study in adolescents with chronic musculoskeletal pain found that pain-related fear was associated with a higher level of disability whereas G-HSD/hEDS was not associated with a higher level of disability. ${ }^{38}$ Whether pain, anticipation of pain, and pain-related fear might indeed contribute more to impaired physical functioning levels in G-HSD/hEDS, as has been found in other chronic pain-conditions ${ }^{39,40}$, should be further explored.

\section{Limitations}

This study has some limitations. The first limitation is a relatively large number of analyses performed in the current study. Therefore, there is an increased risk of a type I error. As mentioned earlier, a second limitation is the small group of adolescents with asymptomatic GJH. However, the prevalence of hypermobility in this study is $18 \%$ which is comparable with other studies performed in a general population, despite the different cut-off points. ${ }^{41,42}$ Further research should be conducted in a larger population. A third limitation is the absence of continuous physical activity registration due to contact sports or swimming. This might result in an underestimation of the participant's actual activity level. Also the lower number of PAL measurements during school and exclusion of 10 adolescents due to not fulfilling the pre-determined criteria for a valid measurement might affect the results. A fourth limitation of this study is the use of the PHODA-youth. The scores on the PHODA-youth and all subscales were low compared to the range. Therefore, it questions the validity of the assessment of perceived harmfulness in a pain-free situation. The PHODA-youth has not been validated in healthy pain-free adolescents yet. Further research is needed to evaluate the assumption that perceived harmfulness can indeed be measured with the PHODA-youth in a pain-free population. The finding that in adults, fear of injury/perceived harmfulness, as assessed 
by the Tampa Scale for Kinesiophobia, can be used in a pain-free population may support the assumption. ${ }^{43}$ The final limitation is the cross-sectional design of the study, which meant no causal relationships could be confirmed. Future studies should employ longitudinal designs to examine associations over time. Evidence has shown that GJH is a risk factor for developing chronic musculoskeletal pain ${ }^{44,45}$ and therefore differences in physical functioning levels before the onset of chronic musculoskeletal pain may help to unravel the influence of GJH on physical functioning levels in adolescents who will or will not develop chronic musculoskeletal pain.

\section{Clinical implications}

This study has clinical implications. A lower level of physical functioning in adolescents with G-HSD/hEDS is not solely caused by GJH. It is therefore important that, in clinical practice, treatment of adolescents with G-HSD/hEDS should also focus on other contributing factors such as pain, fatigue, multi-systemic dysfunction, loss of postural control, and pain-related fear. ${ }^{19,37,46}$

\section{Conclusion}

Adolescents with asymptomatic GJH had no lower level of physical functioning (normal muscle strength, motor performance, and PAL) compared to non-hypermobile controls. Contrary to expectations, knee extensor muscle strength was even significantly increased in adolescents with asymptomatic GJH. The negative impact of perceived harmfulness was not more pronounced in adolescents with asymptomatic GJH. 


\section{References}

1. Beighton P, Grahame R, Bird H. Musculoskeletal Features of Hypermobility and Their Management. . Hypermobility of Joints. 4th ed. London: Springer; 2012:65-99.

2. Murray KJ. Hypermobility disorders in children and adolescents. Best Practice \& Research Clinical Rheumatology. 2006;20(2):329-51.

3. Scheper MC, de Vries JE, de Vos R, Verbunt J, Nollet F, Engelbert RH. Generalized joint hypermobility in professional dancers: a sign of talent or vulnerability? Rheumatology (Oxford). 2013;52(4):651-8.

4. Castori M, Tinkle B, Levy H, Grahame R, Malfait F, Hakim A. A framework for the classification of joint hypermobility and related conditions. American Journal of Medical Genetics Part C 2017;175(1):148-57.

5. Malfait F, Francomano C, Byers P, Belmont J, Berglund B, Black J, et al. The 2017 international classification of the Ehlers-Danlos syndromes. American Journal of Medical Genetics Part $C$. 2017;175(1):8-26.

6. Voermans NC, Knoop H, van de Kamp N, Hamel BC, Bleijenberg G, van Engelen BG. Fatigue is a frequent and clinically relevant problem in Ehlers-Danlos Syndrome. Seminars in Arthritis and Rheumatism. 2010;40(3):267-74.

7. Fatoye F, Palmer S, Macmillan F, Rowe $P$, van der Linden M. Pain intensity and quality of life perception in children with hypermobility syndrome. Rheumatology International. 2012;32(5):1277-84.

8. Engelbert RH, van Bergen M, Henneken T, Helders PJ, Takken T. Exercise tolerance in children and adolescents with musculoskeletal pain in joint hypermobility and joint hypomobility syndrome. Pediatrics. 2006;118(3):690-6.

9. Fatoye F, Palmer S, Macmillan F, Rowe P, van der Linden M. Proprioception and muscle torque deficits in children with hypermobility syndrome. Rheumatology (Oxford). 2009;48(2):152-7.

10. Sahin N, Baskent A, Cakmak A, Salli A, Ugurlu H, Berker E. Evaluation of knee proprioception and effects of proprioception exercise in patients with benign joint hypermobility syndrome. Rheumatology International. 2008;28(10):995-1000.

11. Schubert-Hjalmarsson E, Ohman A, Kyllerman M, Beckung E. Pain, balance, activity, and participation in children with hypermobility syndrome. Pediatric Physical Therapy. 2012;24(4):339-44.

12. Rombaut L, Malfait F, Cools A, De Paepe A, Calders P. Musculoskeletal complaints, physical activity and health-related quality of life among patients with the Ehlers-Danlos syndrome hypermobility type. Disability and Rehabilitation. 2010;32(16):1339-45.

13. Scheper MC, de Vries JE, Juul-Kristensen B, Nollet F, Engelbert RH. The functional consequences of generalized joint hypermobility: a cross-sectional study. BMC Musculoskeletal Disorders. 2014;15:243.

14. Jindal P, Narayan A, Ganesan S, MacDermid JC. Muscle strength differences in healthy young adults with and without generalized joint hypermobility: a cross-sectional study. BMC Sports Science, Medicine and Rehabilitation. 2016;8:12.

15. Scheper M, de Vries J, Beelen A, de Vos R, Nollet F, Engelbert R. Generalized joint hypermobility, muscle strength and physical function in healthy adolescents and young adults. Curr Rheumatol Rev. 2014;10(2):117-25.

16. Jensen BR, Olesen AT, Pedersen MT, Kristensen JH, Remvig L, Simonsen EB, et al. Effect of generalized joint hypermobility on knee function and muscle activation in children and adults. Muscle Nerve. 2013;48(5):762-9.

17. Mebes C, Amstutz A, Luder G, Ziswiler HR, Stettler M, Villiger PM, et al. Isometric rate of force development, maximum voluntary contraction, and balance in women with and without joint hypermobility. Arthritis \& Rheumatism. 2008;59(11):1665-9.

18. Clinch J, Deere K, Sayers A, Palmer S, Riddoch C, Tobias JH, et al. Epidemiology of generalized joint laxity (hypermobility) in fourteen-year-old children from the UK: a population-based evaluation. Arthritis \& Rheumatology. 2011;63(9):2819-27.

19. Leeuw M, Goossens ME, Linton SJ, Crombez G, Boersma K, Vlaeyen JW. The fear-avoidance model of musculoskeletal pain: current state of scientific evidence. Journal of Behavioral Medicine. 2007;30(1): 77-94.

20. Simons LE, Kaczynski KJ. The Fear Avoidance model of chronic pain: examination for pediatric application. The Journal of Pain. 2012;13(9):827-35. 
21. Smits-Engelsman B, Klerks M, Kirby A. Beighton score: a valid measure for generalized hypermobility in children. The Journal of Pediatrics. 2011;158(1):119-23.

22. Juul-Kristensen B, Schmedling K, Rombaut L, Lund H, Engelbert RH. Measurement properties of clinical assessment methods for classifying generalized joint hypermobility-A systematic review. American Journal of Medical Genetics Part C. 2017;175(1):116-47.

23. Stinson JN, Kavanagh T, Yamada J, Gill N, Stevens B. Systematic review of the psychometric properties, interpretability and feasibility of self-report pain intensity measures for use in clinical trials in children and adolescents. Pain. 2006;125(1-2):143-57.

24. Verbunt JA, Nijhuis A, Vikstrom M, Stevens A, Haga N, de Jong J, et al. The psychometric characteristics of an assessment instrument for perceived harmfulness in adolescents with musculoskeletal pain (PHODA-youth). European Journal of Pain. 2015;19(5):695-705.

25. Drouin JM, Valovich-mcLeod TC, Shultz SJ, Gansneder BM, Perrin DH. Reliability and validity of the Biodex system 3 pro isokinetic dynamometer velocity, torque and position measurements. European Journal of Applied Physiology. 2004;91(1):22-9.

26. Rombaut L, Malfait F, De Wandele I, Taes Y, Thijs Y, De Paepe A, et al. Muscle mass, muscle strength, functional performance, and physical impairment in women with the hypermobility type of EhlersDanlos syndrome. Arthritis Care \& Research. 2012;64(10):1584-92.

27. Junge T, Wedderkopp N, Thorlund JB, Sogaard K, Juul-Kristensen B. Altered knee joint neuromuscular control during landing from a jump in 10-15 year old children with Generalised Joint Hypermobility. A substudy of the CHAMPS-study Denmark. Journal of Electromyography and Kinesiology. 2015;25(3):5017.

28. Gustavsson A, Neeter C, Thomee P, Silbernagel KG, Augustsson J, Thomee R, et al. A test battery for evaluating hop performance in patients with an $A C L$ injury and patients who have undergone $A C L$ reconstruction. Knee Surgery, Sports Traumatology, Arthroscopy. 2006;14(8):778-88.

29. Ross MD, Langford B, Whelan PJ. Test-retest reliability of 4 single-leg horizontal hop tests. The Journal of Strength \& Conditioning Research. 2002;16(4):617-22.

30. Verbunt JA, Huijnen IP, Seelen HA. Assessment of physical activity by movement registration systems in chronic pain: methodological considerations. The Clinical Journal of Pain. 2012;28(6):496-504.

31. Ottevaere C, Huybrechts I, De Meester F, De Bourdeaudhuij I, Cuenca-Garcia M, De Henauw S. The use of accelerometry in adolescents and its implementation with non-wear time activity diaries in free-living conditions. Journal of Sports Sciences. 2011;29(1):103-13.

32. Trost SG, Mclver KL, Pate RR. Conducting accelerometer-based activity assessments in field-based research. Medicine \& Science in Sports \& Exercise. 2005;37(11 Suppl):531-43.

33. Huijnen IP, Verbunt JA, Peters ML, Delespaul P, Kindermans HP, Roelofs J, et al. Do depression and pain intensity interfere with physical activity in daily life in patients with Chronic Low Back Pain? Pain. 2010;150(1):161-6.

34. Wilson AC, Palermo TM. Physical activity and function in adolescents with chronic pain: a controlled study using actigraphy. The Journal of Pain. 2012;13(2):121-30.

35. Myers R. Classical and Modern Regression with Applications. Belmont: Duxbury Press; 1990.

36. Schmidt $H$, Pedersen TL, Junge T, Engelbert R, Juul-Kristensen B. Hypermobility in Adolescent Athletes: Pain, Functional Ability, Quality of Life, and Musculoskeletal Injuries. The Journal of Orthopaedic and Sports Physical Therapy. 2017;47(10):792-800.

37. Scheper MC, Nicholson LL, Adams RD, Tofts L, Pacey V. The natural history of children with joint hypermobility syndrome and Ehlers-Danlos hypermobility type: a longitudinal cohort study. Rheumatology (Oxford). 2017;56(12):2073-83.

38. van Meulenbroek T, Huijnen IPJ, Wiertz CMH, Verbunt JA. Pain-Related Fear and Its Disabling Impact in Hypermobile Adolescents With Chronic Musculoskeletal Pain. The Journal of Orthopaedic and Sports Physical Therapy. 2017;47(10):775-81.

39. Martin AL, McGrath PA, Brown SC, Katz J. Anxiety sensitivity, fear of pain and pain-related disability in children and adolescents with chronic pain. Pain Research and Management. 2007;12(4):267-72.

40. Cohen LL, Vowles KE, Eccleston C. The impact of adolescent chronic pain on functioning: disentangling the complex role of anxiety. The Journal of Pain. 2010;11(11):1039-46. 
41. Remvig L, Jensen DV, Ward RC. Epidemiology of general joint hypermobility and basis for the proposed criteria for benign joint hypermobility syndrome: review of the literature. The Journal of Rheumatology. 2007;34(4):804-9.

42. Scheper MC, Engelbert RH, Rameckers EA, Verbunt J, Remvig L, Juul-Kristensen B. Children with generalised joint hypermobility and musculoskeletal complaints: state of the art on diagnostics, clinical characteristics, and treatment. BioMed Research International. 2013;2013:121054.

43. Houben RM, Leeuw M, Vlaeyen JW, Goubert L, Picavet HS. Fear of movement/injury in the general population: factor structure and psychometric properties of an adapted version of the Tampa Scale for Kinesiophobia. Journal of Behavioral Medicine. 2005;28(5):415-24.

44. Sohrbeck-Nohr O, Kristensen JH, Boyle E, Remvig L, Juul-Kristensen B. Generalized joint hypermobility in childhood is a possible risk for the development of joint pain in adolescence: a cohort study. BMC Pediatrics. 2014;14:302.

45. Tobias JH, Deere K, Palmer S, Clark EM, Clinch J. Joint hypermobility is a risk factor for musculoskeletal pain during adolescence: findings of a prospective cohort study. Arthritis \& Rheumatology. 2013;65(4):1107-15.

46. Vlaeyen JW, Linton SJ. Fear-avoidance and its consequences in chronic musculoskeletal pain: a state of the art. Pain. 2000;85(3):317-32. 


\section{Chapter 5}

Are chronic musculoskeletal pain and generalized joint hypermobility disabling contributors to physical

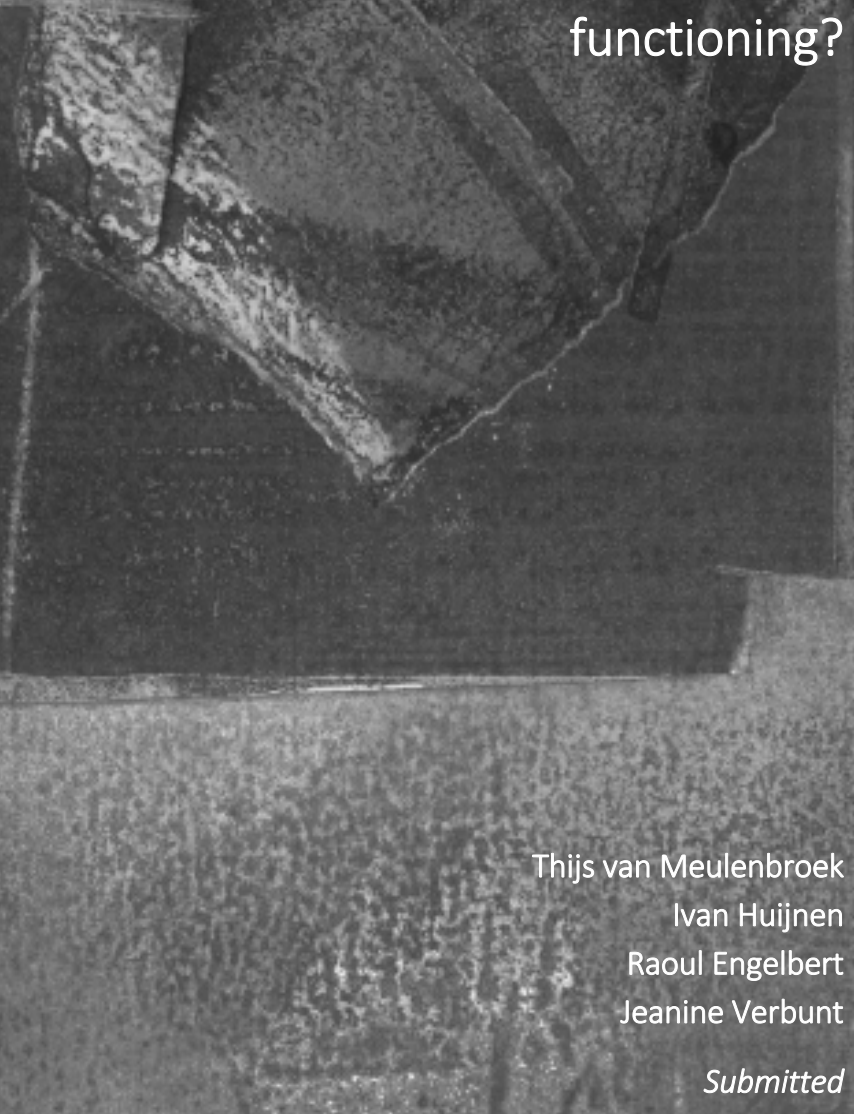




\section{Abstract}

\section{Background and aim}

Chronic musculoskeletal pain (CMP), generalized joint hypermobility $(\mathrm{GJH})$, and pain-related fear have influence on physical functioning in adolescents.

To evaluate differences in physical functioning between adolescents with CMP, GJH or the combination of both, and in addition evaluate the potential contribution of pain-related fear.

\section{Design and setting}

Observational, cross-sectional design.

The adolescents with CMP were recruited by a physician in rehabilitation medicine and measured in the university outpatient rehabilitation clinic (Adelante/Maastricht University Medical Center+, the Netherlands). The adolescents without CMP were recruited in the Southern area of the Netherlands and measured in the university outpatient rehabilitation clinic (Adelante/Maastricht University Medical Centert, the Netherlands).

\section{Population}

Four subgroups of adolescents were included; 21 adolescents with CMP without GJH, 9 adolescents with $\mathrm{CMP}$ and $\mathrm{GJH}, 51$ adolescents without $\mathrm{CMP}$ without $\mathrm{GJH}$, and 11 adolescents without CMP with GJH.

\section{Methods}

Outcome measures were muscle strength and endurance, motor performance, physical activity level, and pain-related fear. Hierarchical regression analyses were used to study differences in physical functioning and the contribution of pain-related fear in adolescents with/without CMP as well as with/without GJH.

\section{Results}

Adolescents with CMP had decreased muscle strength ( $p=0.01$ ), endurance $(p=0.02)$, and lower motor performance $(p<0.01)$ compared to adolescents without CMP. Higher levels of pain-related fear were related to decreased muscle strength $(p=0.01)$, endurance $(p<0.01)$, and motor performance $(p<0.01)$. No differences in physical functioning and pain-related fear between hypermobile and non-hypermobile adolescents with CMP were found.

\section{Conclusions}

Adolescents with CMP had decreased muscle strength and motor performance associated with increased levels of pain-related fear compared to adolescents without CMP. The association of being hypermobile with physical functioning is not more pronounced in adolescents with CMP.

\section{Clinical rehabilitation impact}

No differences were found in physical functioning and pain-related fear between hypermobile adolescents with CMP compared to non-hypermobile adolescents with CMP. Future rehabilitation treatment in hypermobile adolescents with CMP should also focus on psychological components, such as pain-related fear. 


\section{Introduction}

Pain lasting for more than 3 months is referred to as chronic pain. ${ }^{1}$ Chronic pain is a complex health issue in which physiological, psychological, and social factors contribute to the level of pain-related disability. ${ }^{2}$ In the Netherlands, up to $25 \%$ of the schoolchildren report chronic pain, such as headache, abdominal pain or chronic musculoskeletal pain (CMP). ${ }^{3}$ In CMP, often a specific medical disease cannot explain the level of disability, resulting in a non-specific condition based on the location of symptoms in the bones, joints, muscles or related soft tissues. ${ }^{4}$ In approximately $40 \%$ of adolescents with CMP, pain has a considerable disabling impact on physical functioning (associated with deficits in motor performance, difficulty with balance or decreased muscle strength), mood, and social functioning, reducing the quality of life.,

Previous studies suggest a relationship between $\mathrm{CMP}$ and generalized joint hypermobility $(\mathrm{GJH})$, as GJH has been reported as a two to threefold increased risk of developing CMP.,8 GJH associated with one or more musculoskeletal manifestations such as chronic widespread pain for $\geq 3$ months, macro - and microtrauma, disturbed proprioception, and joint instability, is referred to as generalized hypermobility spectrum disorder (G-HSD). ${ }^{9}$ G-HSD shows close overlap with the hypermobile Ehlers-Danlos syndrome (hEDS). ${ }^{10}$ Both symptomatic conditions lack a specific genetic profile, have been identified as clinically indistinguishable ${ }^{11}$ and are therefore labelled as GHSD/hEDS.

Common physical symptoms in children and adolescents with G-HSD/hEDS that have been reported are decreased muscle strength ${ }^{12}$, reduced cardiorespiratory fitness ${ }^{13}$, impaired proprioception ${ }^{12}$, and reduced balance. ${ }^{14}$ Furthermore, children and adolescents reported decreased level of participation in activities. ${ }^{14}$ Deconditioning has been reported as a potential underlying explanation for decreased functioning in musculoskeletal pain. According to the fear-avoidance model, musculoskeletal pain can be perceived as a threat, and pain-related fear can evolve causing avoidance behaviour. This pain-related behaviour might result in disuse, functional disability, and depression, resulting in deconditioning further fuelling the vicious circle of CMP. Even more, individuals with G-HSD/hEDS have been found to report higher levels of pain-related fear ${ }^{15,16}$ and more generalized anxiety. ${ }^{17}$ It is hypothesized that this vulnerability for heightened fear and anxiety might lead to further disabilities and thus a further decline in physical functioning. In earlier studies, it was found that adolescents with asymptomatic, thus pain-free, GJH have decreased functional capacity, expressed as a decrease in walking distance and jumping capacity ${ }^{18}$, decreased muscle strength ${ }^{19}$, and preferring more stable activities, such as walking and cycling. ${ }^{19}$ Other studies could not confirm the altered physical activity level and reduced muscle strength in $\mathrm{GJH}^{20,21}$ 
Thus, it remains still unclear whether having CMP, being hypermobile in the joints, having the combination of both, or the contributing role of pain-related fear will have the most impact on physical functioning. Therefore, the main aim of this study was to evaluate the influence of GJH and having CMP on the level of physical functioning (measured as muscle strength, motor performance, and physical activity level [PAL]) in adolescents. We hypothesized that having CMP and GJH will negatively interact on physical functioning in adolescents than CMP alone. Furthermore, we want to examine the specific role of pain-related fear on physical functioning. We hypothesize that higher levels of pain-related fear in adolescents with G-HSD/hEDS are related to decreased levels of physical functioning compared to adolescents with CMP.

\section{Materials and methods}

\section{Participants and procedure}

In this cross-sectional study both adolescents with CMP and healthy adolescents participated. First, thirty adolescents (mean age 16.4 years, SD 1.7 years, range 13-20 years) with CMP and GJH (+/+) or with CMP and without GJH (+/-) were recruited by a physician in rehabilitation medicine in the university outpatient rehabilitation clinic specialised in pain rehabilitation (Adelante/Maastricht University Medical Centert, the Netherlands). Inclusion criteria were a treatment indication for outpatient rehabilitation treatment, considerable perceived disabilities in daily life functioning, and good understanding of the Dutch language. Exclusion criteria were any suspicion of a medical (orthopaedic, rheumatic neurological) disease that could fully explain their current level of pain or any suspicion of an underlying psychiatric disorder that would hamper rehabilitation treatment. The adolescents with CMP were participants in the multicenter 2B-Active trial (NL47323.068.13), which was conducted in Adelante/Maastricht University Medical Center+, Laurentius Hospital Roermond, Revant Rehabilitation Center Breda, and Rijndam Rehabilitation Center in Rotterdam. Ethical approval was granted by the Medical Ethics Committee of the Academic Hospital Maastricht/Maastricht University, the Netherlands (METC; 13-3-062) in accordance with the principles set forth in the Helsinki Declaration in October 2013. The 2B-Active study investigated the effectiveness of a multidisciplinary rehabilitation treatment including exposure in vivo treatment in reducing functional disability in adolescents with CMP compared with care as usual. Baseline measurements including physical functioning measurements were completed before the start of the outpatient rehabilitation treatment. On a single study site (Adelante/Maastricht University Medical Center+) baseline measurements also included assessment on physical functioning. For study site Maastricht, 62 participants 
were anticipated for in 2B-Active. ${ }^{22}$ Only 30 adolescents could eventually be included in the current study.

Second, anticipating on 62 adolescents with CMP to participate, also 62 healthy adolescents (mean age 16.8 years, SD 2.3 years, range $12-21$ years) without CMP with GJH (-/+) or without CMP and without GJH (-/-) were recruited in the Southern area of the Netherlands. The inclusion of the adolescents without CMP with GJH $(-/+)$ and without CMP and without GJH (-/-) was in the same time period to the adolescents with CMP and GJH (+/+) and with CMP and without GJH (+/-) (Figure 5.1). Therefore, the composition of the adolescent population without CMP was based on age and gender of the adolescents with CMP. Recruitment of these healthy adolescents was organized in three different ways: (1) informed on a local high school, (2) responded on a pamphlet presented at two institutes for higher education, and (3) asked in the personal network of the research group. Adolescents who were interested received an information letter and after a week, a researcher contacted them for participating in the study. Inclusion criteria were good understanding of the Dutch language and adolescents were excluded if there was a specific medical condition influencing physical functioning, such as acute or recurrent musculoskeletal pain or a history of surgical interventions. Ethical approval was granted by the Medical Ethics Committee of the Academic Hospital Maastricht/Maastricht University, the Netherlands (METC; 15-4-052) in accordance with the principles set forth in the Helsinki Declaration in October 2013. All adolescents and, if younger than 18 years of age, also from their parents provided written informed consent.

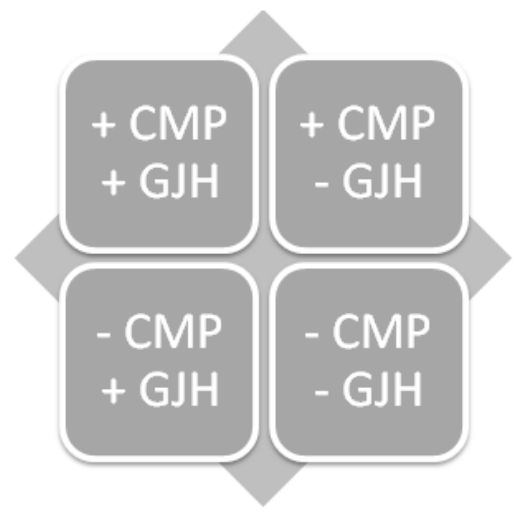

Figure 5.1 Explaining the different groups.

Abbreviations: + CMP + GJH = adolescents with chronic musculoskeletal pain and generalized joint hypermobility; + CMP - GJH = adolescents with chronic musculoskeletal pain and no generalized joint hypermobility; $-\mathrm{CMP}+\mathrm{GJH}=$ healthy adolescents with asymptomatic generalized joint hypermobility; - CMP - GJH = healthy adolescents without asymptomatic generalized joint hypermobility. 
All outcome measures were collected by a physical therapist blinded for the condition during an 1-hour session. Assessment of the adolescents with CMP and with or without GJH were at baseline prior to the start of their outpatient rehabilitation treatment. Healthy adolescents without CMP and with or without GJH were invited to come to the hospital once in order to participate in the study assessment procedure.

\section{Outcome measurements}

Sociodemographic variables (age and gender) were collected. Height (meters, m) and weight (kilograms, $\mathrm{kg}$ ) were measured in a standardized method without heavy clothing and shoes. Body mass index $\left(\mathrm{BMI}, \mathrm{kg} / \mathrm{m}^{2}\right)$ was calculated as weight divided by the square of height. Perceived difficulty in performing activities at school, at home, and in recreational or social interactions was assessed with the Functional Disability Inventory (FDI) in the adolescents with CMP. The FDI is a reliable and valid instrument to evaluate pain-related disability in adolescents. ${ }^{23}$

\section{Hypermobility}

The presence of joint hypermobility was assessed using Beighton score (BS), with a standardized protocol. The BS consist out of nine functional tests and scored dichotomously (0/1) with a maximum score of nine. The cut-off point for adolescents younger than 18 years is 6 out of 9 and the cut-off point for adolescents older than 18 years is 5 out of $9 .^{24}$ The BS is the most commonly used method in clinical practice. ${ }^{24}$ In the adolescents with CMP, a physician in rehabilitation medicine measured GJH using BS. This protocol was also used by a physical therapist to measure GJH in the adolescents without CMP.

\section{Physical functioning}

In order to reflect various components of physical functioning: muscle strength, muscle strength endurance, motor performance, and physical activity in daily life (PAL) were assessed.

\section{Muscle strength}

For measuring isokinetic knee extensor and knee flexor muscle strength in both legs, a dynamometer (Biodex System 3 Pro dynamometer, Biodex Medical Systems, Shirley, NY, USA) was used. Knee muscle strength is an important factor in balance and functional activities of daily living, such as standing up, stairs climbing, and gait. ${ }^{25}$ The adolescent took place in an upright sitting position and the tested upper leg was stabilized with a fixation strap. The lever arm was attached to the adolescent's lower leg by a padded cuff 
$2 \mathrm{~cm}$ proximal to the medial malleolus. The axis of movement of the knee extension/flexion was in line with the axis of movement of the Biodex. After one practice repetition, five repetitions of maximal voluntary concentric knee extension and flexion were performed at the angular velocity of $60 \%$ second. Peak torque/body weight (PT/BW) was used to standardize and interpret isokinetic muscle strength.

The same setup was used to measure isokinetic knee extensor and knee flexor muscle strength endurance in both legs. The adolescent had to perform 30 repetitions of maximal voluntary concentric knee extension and flexion with an angular velocity of $240 \%$ second after one practice repetition. The test was performed 60 seconds after the isokinetic test of $60 \%$ second. Total work (J) was used to represents the muscle's capacity. The Biodex is a valid and reliable isokinetic dynamometer in adolescents. ${ }^{26}$

\section{Motor performance}

For measuring motor performance related to balance, coordination, and joint stability, the single leg hop for distance (SLHD) was used. ${ }^{27}$ The adolescents were asked to jump as far as possible on a single leg, without losing balance. After one practice hop, three valid hops with both legs were performed with a resting period of 30 seconds in between. The greatest distance (centimetres) from the toe at the push off to the heel at the place the adolescents landed was used for the analyses. The SLHD has shown high intra-subject reliability. ${ }^{28}$

\section{Physical activity in daily life (PAL)}

PAL during daily life was measured using a tri-axial accelerometer (AX3, Axivity, Newcastle, UK) attached to the waist using plaster (Tegaderm Film, 10x12 cm). The AX3 had to be removed during potential harmful activities for the device, such as swimming and contact sports. Each adolescent received a diary to collect information of wake up time, sleeping, and non-wearing time and the reason for doing this. To be included as a valid score, at least three weekdays with a minimum of 10 hours of recording and one weekend day with at least 8 hours of recording had to be available during a 7-day monitoring period. ${ }^{29}$ To obtain activity counts an algorithm was designed using Matlab (The Math Works Inc., Natick, MA, USA), based on the method which was used for the Actiwatch 7 (Philips, Eindhoven, the Netherlands). The highest recorded sample per minute was selected and summed per minute. This counts/minute signal was used for all further calculations. Daily uptime ( $\mathrm{min}$ ) was defined as the period between wake up time and sleeping. PAL was expressed as total activity (TA) during uptime. This quantity was calculated as the total sum of counts during uptime averaged over the week. ${ }^{30}$ 


\section{Pain intensity}

Pain intensity was measured using a 100-mm visual analogue scale (VAS), ranging from 0 (no pain) to 100 (worst pain imaginable). The adolescents rated their pain intensity in three conditions: (1) current pain, (2) the worst/most severe pain experienced in the last week, and (3) the least pain experienced in the last week. The mean of these three VAS scores was used. The VAS is a reliable and valid measurement in children older than 8 years. $^{31}$

\section{Perceived harmfulness}

Perceived harmfulness, as construct of pain-related fear, was measured with the Photograph Series of Daily Activities for youth (PHODA-youth). The PHODA-youth contains 51 age-specific activities and social situations and consists out of three categories: activities of daily living and household (PHODA-ADL; 13 items), intensive physical activities (PHODA-PA; 27 items), and social activities (PHODA-SA; 11 items). Adolescents rate the photographs from 0 (not harmful at all) to 10 (extremely harmful), where higher scores indicated higher levels of perceived harmfulness. The PHODA-Youth is valid and reliable in adolescents with CMP. ${ }^{32}$

\section{Statistical analysis}

Normality of the data and the presence of outliers was checked by visual assessment and the Kolgomorov-Smirnov goodness-of-fit test. Descriptive data of sociodemographic and anthropometric variables, hypermobility, functional disability (CMP group only), and pain intensity of the CMP group with/without GJH and the non-CMP with/without GJH were calculated. Normal distribution of data were presented as mean \pm standard deviation (SD), non-normal distribution data were presented as median and interquartile range (IQR). Categorical data were shown as frequencies.

In order to study differences in physical functioning and pain-related fear measured as perceived harmfulness in adolescents with both $\mathrm{CMP}$ and $\mathrm{GJH}$, adolescents with CMP without GJH, adolescents without CMP with GJH, and those without CMP nor GJH multiple one-way ANOVA's were used in case of a normal distribution. In case of a significant result, a post-hoc Hochberg's GT2 analysis was performed to explore potential differences between groups. In case of non-normal distribution, the KruskallWallis test was used. Subsequent multiple comparisons included Mann-Whitney $U$ tests. For the categorical variables, the Fisher's Exact test was used.

In order to evaluate the influence of having GJH and having CMP on the level of physical functioning (muscle strength, motor performance, and PAL) in adolescents, and to examine the specific role of pain-related fear on physical functioning, hierarchical 
regression analyses were performed. Variables were entered in three steps. In the first step, physical functioning (respectively expressed as muscle strength (1), muscle strength endurance (2), motor performance (3), and physical activity level (4)) were the dependent variables. In each model having CMP (no=0; yes=1), GJH (no=0; yes=1), age, and gender (male=0; female=1) were entered as independent variables. In the second step, pain-related fear (measured as perceived harmfulness) was introduced. In the third step, the interaction term (CMP $\times$ GJH) was entered. In case of a significant interaction $(p<0.10)$, additional regression analyses were performed for the CMP group and the non-CMP group separately with the physical functioning outcome as dependent variable and GJH, age, gender, and pain-related fear as independent variables.

For all independent variables, the association with the outcome was presented by the regression coefficient (B), the corresponding 95\% confidence interval (95\%-Cl), and $p$-value. It appeared that knee extension and knee flexion muscle strength endurance did not meet the assumption of normality; these variables were log transformed prior to the analyses. After transformation, skewness and kurtosis values of the scales were in acceptable range of -1 and +1 . For all regression analyses, a collinearity check was performed. Collinearity was considered a problem if the variance inflation factor (VIF) was above $10 .{ }^{33}$ P-values $<0.05$ were considered statistically significant. All statistical analyses were performed in IBM SPSS Statistics for Windows V23.0 (IBM Corp, Armonk, NY, USA).

\section{Results}

\section{Description of the population}

A total of 92 adolescents, 30 adolescents with CMP and 62 adolescents without CMP, participated in the present study. Within the CMP group 9 of 30 adolescents had GJH (28\%) and within the non-CMP group 11 of 62 adolescents had GJH (18\%). The characteristics of each group are presented in Table 5.1. The mean score of the FDI was respectively $24.3 \pm 8.0$ in the adolescents with CMP and without GJH (+/-) and $22.1 \pm 13.4$ (not significant) in the adolescents with CMP and GJH $(+/+)$, which is classified as moderate disability (score 13-29). ${ }^{23}$ With respect to gender, age, height, weight, and $\mathrm{BMI}$ no significant differences were found between the four groups (CMP without GJH (+/-), CMP with GJH (+/+), without CMP without GJH (-/-), and without CMP with GJH $(-/+))$. As expected, the Beighton score was significant higher $(p<0.01)$ in the $\mathrm{CMP}$ with GJH and the non-CMP with GJH compared to the CMP without GJH and the non-CMP without GJH, and pain intensity was significant higher $(p<0.01)$ in the CMP with and without GJH compared to the non-CMP with and without GJH. 
Table 5.1 Characteristics of the study population of adolescents with and without CMP and with and without GJH.

\begin{tabular}{|c|c|c|c|c|c|}
\hline & \multicolumn{2}{|c|}{$\mathrm{CMP}(\mathrm{n}=30)$} & \multicolumn{2}{|c|}{ No CMP (n=62) } & \multirow[b]{2}{*}{ Test statistics } \\
\hline & No GJH $(n=21)$ & GJH $(n=9)$ & No GJH $(n=51)$ & GJH $(n=11)$ & \\
\hline Female/male, $n$ & $19 / 2$ & $8 / 1$ & $37 / 14$ & $10 / 1$ & $p=0.27$ \\
\hline Age, y & $16.1 \pm 2.0$ & $16.9 \pm 0.8$ & $16.5 \pm 2.3$ & $18.1 \pm 1.8$ & $\begin{array}{c}\mathrm{F}_{3,88}=2.36 \\
p=0.08\end{array}$ \\
\hline Height, m & $1.66 \pm 0.08$ & $1.71 \pm 0.03$ & $1.70 \pm 0.09$ & $1.70 \pm 0.10$ & $\begin{array}{c}\mathrm{F}_{3,88}=0.99 \\
p=0.40\end{array}$ \\
\hline Weight, kg & $60.5(57.3-72.8)$ & $80.4(56.6-110.3)$ & $59.0(53.1-69.7)$ & $63.1(52.0-73.7)$ & $\begin{array}{c}\mathrm{H}_{3}=6.12 \\
p=0.11\end{array}$ \\
\hline $\mathrm{BMI}, \mathrm{kg} / \mathrm{m}^{2}$ & $22.1(21.0-27.0)$ & $27.6(19.8-37.1)$ & $21.5(18.9-23.0)$ & $22.1(18.5-24.2)$ & $\begin{array}{c}\mathrm{H}_{3}=7,36 \\
p=0.06\end{array}$ \\
\hline Beighton, $\mathrm{n}$ & $2(1-3)^{a}$ & $6(6-7)^{b}$ & $2(2-3)^{a}$ & $6(5-7)^{b}$ & $\begin{array}{c}\mathrm{H}_{3}=47.86 \\
p<0.01\end{array}$ \\
\hline Pain intensity, mm & $53(38-77)^{a}$ & $68(37-78)^{a}$ & $3(0-11)^{b}$ & $5(0-9)^{b}$ & $\begin{array}{c}\mathrm{H}_{3}=51.66 \\
p<0.01\end{array}$ \\
\hline Functional disability & $24.3 \pm 8.0^{c}$ & $22.1 \pm 13.4^{d}$ & - & - & $\begin{aligned} t_{25} & =0.40 \\
p & =0.70\end{aligned}$ \\
\hline
\end{tabular}

Abbreviation: $\mathrm{BMI}=$ body mass index. Normally distributed data are presented by mean \pm SD and not normally distributed data are presented by median (interquartile ranges; $25-75^{\text {th }}$ percentile). Functional disability is only measured in the CMP population. ${ }^{a, b}$ Values with the same superscripts represent homogenous subsets. ${ }^{c} \mathrm{n}=20$. ${ }^{d} n=7$.

\section{Physical functioning and pain-related fear}

One non-CMP adolescent without GJH could not complete the muscle strength endurance testing due to malfunction of the dynamometer. Three adolescents with CMP (2 with GJH and 1 without GJH) did not complete the PHODA-youth. For the assessment of PAL, accelerometry data of 75 adolescents (26 adolescents with CMP; 49 adolescents without CMP) was used. Data of 17 adolescents (4 adolescents with CMP; 15 adolescents without CMP) were excluded due to the following reasons: dysfunction of the accelerometer $(n=4)$, not fulfilling the pre-determined criteria for a valid registration $(n=12)$, and interruption of the measurement due to an allergic reaction to the plaster $(n=1)$. Table 5.2 presents mean $\pm S D$ and median scores (IQR) on the four components of physical functioning and pain-related fear of the four subgroups. The subgroups CMP with/without GJH scored significantly higher on pain-related fear compared to non-CMP with/without GJH $(p<0.01)$. No differences in pain-related fear was found between the CMP with GJH and the CMP without GJH ( $p=0.38)$. 


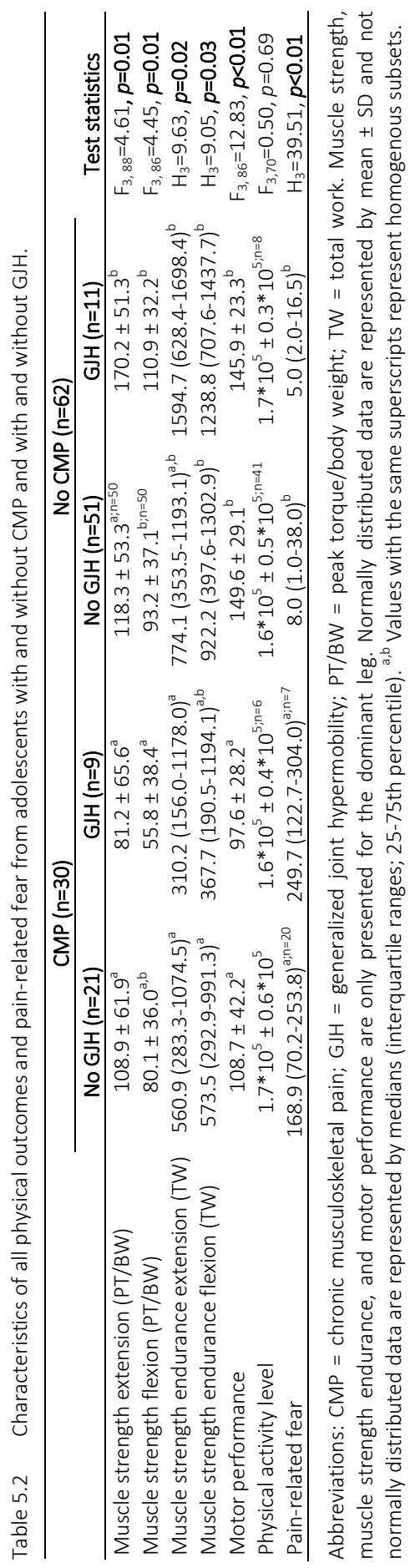




\section{Associations between having CMP and GJH, pain-related fear, and physical functioning}

\section{Muscle strength}

Table 5.3 presents the results of the hierarchical regression analyses with muscle strength as dependent variable. The first step showed that older adolescents had increased knee extension muscle strength $(B=7.58, p=0.01)$ and having CMP was associated with decreased knee flexion muscle strength $(B=-22.88, p=0.01)$. In the second step, the PHODA-youth was associated with knee extensor $(B=-0.24, p=0.01)$ and flexor $(B=-0.17, p=0.01)$ muscle strength, indicating that higher perceived harmfulness was associated with decreased muscle strength. In the final step, the interaction term CMP $\times$ GJH was introduced. There was a significant association $(p<0.10)$ with decreased knee extensor muscle strength $(p=0.04)$ and knee flexor muscle strength $(p=0.07)$. The analysis per subgroup (CMP group and non-CMP group) revealed that higher painrelated fear was associated with decreased knee extensor $\left(B=-0.25, p=0.02 ; R^{2}=0.28\right)$ and flexor muscle strength $\left(B=-0.15, p=0.01 ; R^{2}=0.33\right)$ in adolescents with CMP. No significant association was found for having GJH in the CMP group (muscle strength extension: $\mathrm{B}=-19.60, p=0.48$; muscle strength flexion: $\mathrm{B}=-20.74, p=0.19)$. In the non-CMP group having $\mathrm{GJH}(\mathrm{B}=41.05, p=0.03)$ was associated with increased knee extensor muscle strength (corresponding $R^{2}=0.21$ ).

\section{Muscle strength endurance}

For muscle strength endurance the first step showed that having CMP was associated with decreased knee extension $(B=-0.20, p=0.02)$ and decreased knee flexion $(B=-0.20$, $p<0.01)$. Furthermore, a higher age was associated with increased knee extension $(\mathrm{B}=0.04, p=0.04)$ and increased knee flexion $(\mathrm{B}=0.03, p=0.05)$ muscle strength endurance. After adding perceived harmfulness in step 2, significant associations were found with knee extension $(B<-0.01, p<0.01)$ and knee flexion $(B<-0.01, p<0.01)$ muscle strength endurance. In the final step, the interaction term CMP $x$ GJH was not significantly associated with knee extensor and knee flexor muscle strength endurance (Table 5.3). 


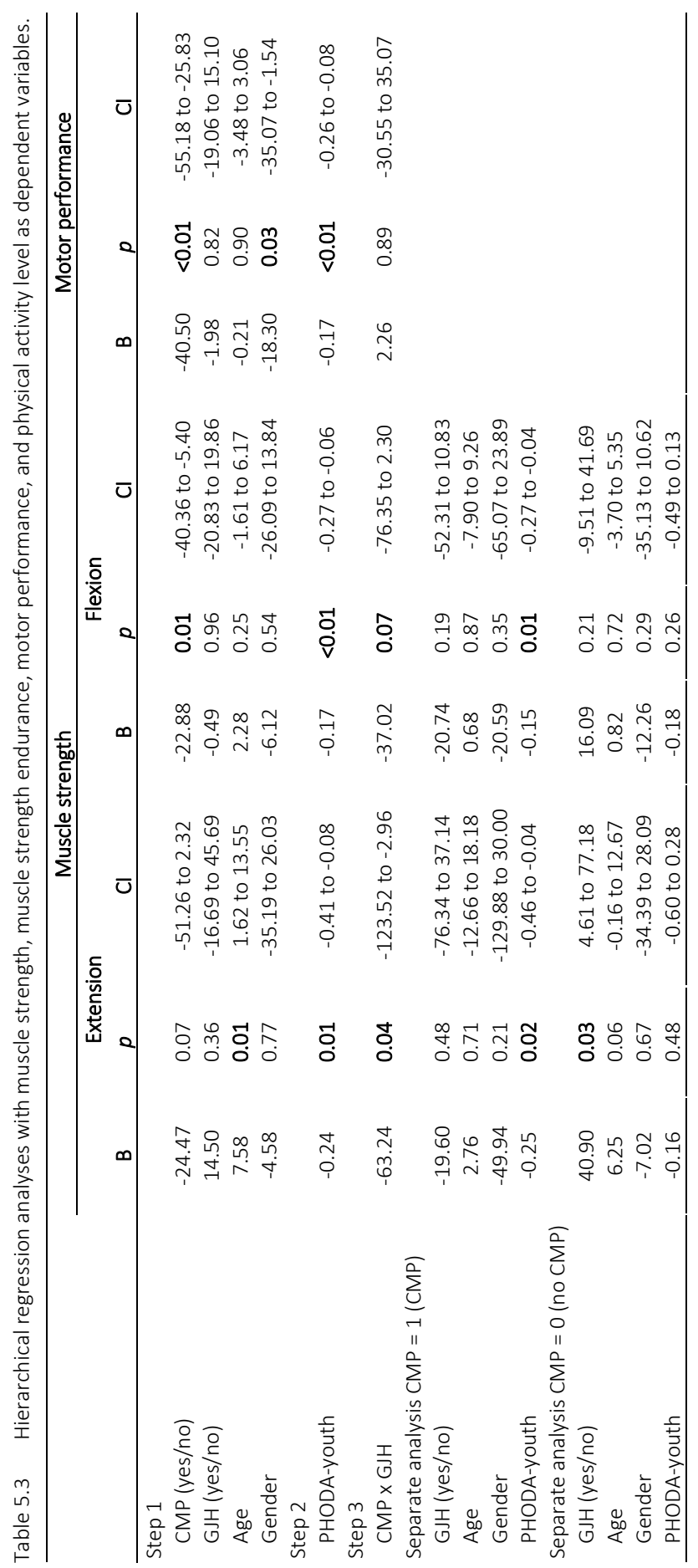




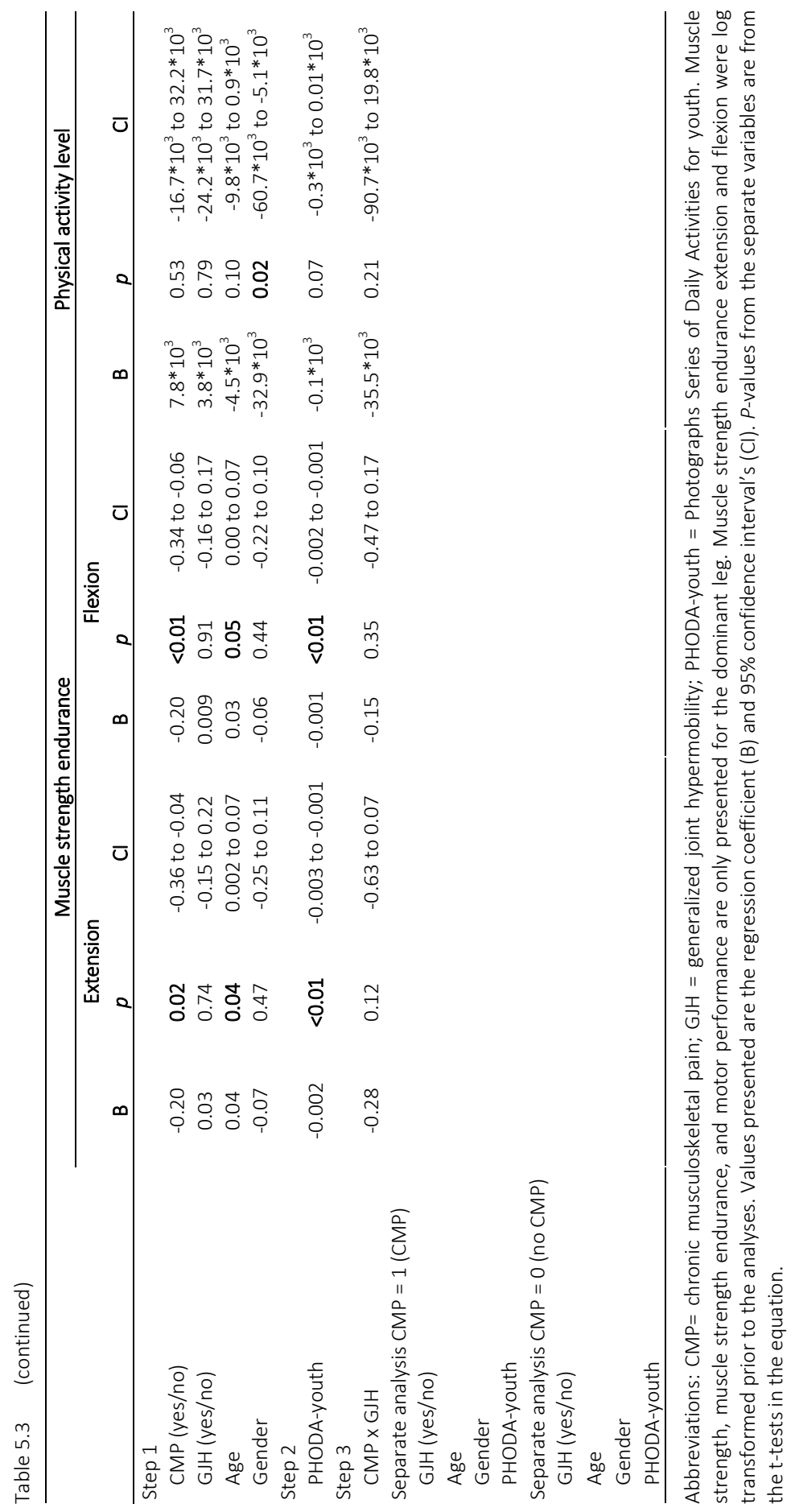




\section{Motor performance}

The results in the first step for motor performance showed that after adding having $\mathrm{CMP}, \mathrm{GJH}$, age, and gender only having CMP was associated with decreased motor performance $(B=-40.50, p<0.01)$ and male adolescents had a significant higher score on the SLHD ( $B=18.30, p=0.03)$. In the second step, perceived harmfulness was associated with motor performance, indicating that higher scores of perceived harmfulness were associated with decreased motor performance $(B=-0.17, p<0.01)$. In the third step, the interaction term CMP $\times$ GJH was added, but no significant association was found (Table 5.3).

\section{Physical activity level}

For PAL the first step showed that female adolescents had decreased levels of PAL $\left(\mathrm{B}=-32.9 * 10^{3}, p=0.02\right)$. No significant association was found for having CMP. After adding perceived harmfulness in the second step and the interaction term CMP $\times$ GJH in the third step, no significant associations were found (Table 5.3).

\section{Discussion}

This study showed that adolescents with CMP had decreased muscle strength, decreased muscle strength endurance, and lower motor performance compared to adolescents without CMP. In contrast to our expectations, the results indicated that adolescents with G-HSD/hEDS do not have lower physical functioning levels compared to non-hypermobile adolescents with CMP. Furthermore, this study could not confirm lower objectively PAL in adolescents with CMP (with/without GJH) compared to adolescents without CMP (with/without GJH). In addition, higher levels of pain-related fear, which was found in adolescents with CMP, were associated with decreased muscle strength, muscle strength endurance, and motor performance. However, in contrast to our expectations no difference between the influence of pain-related fear on physical functioning in adolescents with G-HSD/hEDS compared to non-hypermobile adolescents with CMP was found.

The findings of the current study were consistent with results of previous studies who found decreased abdominal and back muscle strength endurance and a reduction in motor performance, using the drop vertical jump test, in adolescents with CMP compared to pain-free controls. ${ }^{4,34,35}$ Furthermore, other studies also showed decreased knee flexor and extensor muscle strength and reduced motor performance in children and adolescents with G-HSD/hEDS compared to healthy controls. ${ }^{12,14}$ In addition, the current study used the SLHD as dynamic performance test determined by coordination, 
balance, and joint stability ${ }^{27}$, whereas Schubert-Hjalmarsson et al. ${ }^{14}$ measured motor performance with another performance test (Bruininks-Oseretsky test of motor proficiency). However, the results of the current study did not support the hypothesized negative impact of having GJH and CMP on physical functioning compared to CMP alone. A possible explanation for these findings could be that the selected CMP population in this study was included in an university outpatient rehabilitation clinic specialised in pain rehabilitation and already experiencing long-term disabling pain complaints resulted in a decline of daily functioning and deconditioning in both the hypermobile and nonhypermobile adolescents with CMP. This deconditioning may have resulted in decreased muscle strength, decreased muscle strength endurance, and lower motor performance in hypermobile and non-hypermobile adolescents with CMP compared to adolescents without CMP, but no differences occurred between the hypermobile and nonhypermobile adolescents with CMP. These results are based on only a small number of adolescents with G-HSD/hEDS ( $n=9$ ) and should therefore be treated with caution. An unexpected finding was that there were no differences in objectively measured PAL between the different subgroups. This was in contrast with other studies showing lower levels of objectively assessed and subjectively reported PAL in adolescents with CMP or G-HSD/hEDS compared to healthy adolescents. ${ }^{14,36,37}$ However, another study also found similar subjective PAL in adolescents with and without CMP. ${ }^{38}$ In addition, the studies of Stommen et al. ${ }^{38}$ and Schubert-Hjalmarsson et al. ${ }^{14}$ showed that pain-free adolescents appeared to be more active in sports or outdoor games. This last finding could also be of interest in the current population, though due to the absence of continuous physical activity measurements during contact sports and/or swimming we could not differentiate between the different intensities of PAL. Thus, it appeared that adolescents with CMP and with/without GJH do not differ in PAL compared to adolescents without CMP and with/without GJH, despite the decreased muscle strength and lower motor performance. The findings of the decreased muscle strength and lower motor performance might be influenced by pain experienced during testing and psychological factors, such as pain catastrophizing and pain-related fear. Adolescents with CMP might decide to stop or perform submaximal, resulting in a lower score. ${ }^{39}$ Therefore, in future studies it is important to distinguish whether physical deconditioning or submaximal performance due to pain and/or pain-related fear is accounting for the physical outcome.

Furthermore, the results of the current study showed that pain-related fear in adolescents with CMP, independent from having GJH or not, is associated with decreased muscle strength, muscle strength endurance, and motor performance. This supports previous findings of the growing evidence on the negative association of painrelated fear on disability in adolescents with $\mathrm{CMP} .{ }^{40,41}$ Furthermore, other studies in 
subjects with hEDS also showed heightened fear of pain and movement, using the Tampa Scale of Kinesiophobia. ${ }^{15,17}$ In addition, Rombaut et al. ${ }^{42}$ reported increased fear of falling in adults with hEDS compared to pain-free non-hypermobile controls, which might contribute to further muscle weakness and postural instability. However, as mentioned before, pain-related fear could also lead to a submaximal performance on the physical outcome tests resulting in a lower score. In addition, there were no differences in pain-related fear between adolescents with G-HSD/hEDS compared to non-hypermobile adolescents with CMP. This finding supported a previous study who also found no differences in pain-related fear, measured with the Fear of Pain Questionnaire-Child report between hypermobile and non-hypermobile adolescents with CMP. ${ }^{43}$

In 2017 a consensus statement with new nosology according to unified criteria is published and the terms G-HSD/hEDS replaced the old nomenclature of joint hypermobility syndrome and Ehlers-Danlos syndrome hypermobility type., ${ }^{9,10}$ In this study we used G-HSD/hEDS as an umbrella term to cover the new and old nomenclature of GJH-related disorders with CMP. It should be noted that it is currently unknown what the consequences are of these new unified criteria for patient outcomes and disease distinctive.

To our knowledge this was the first study focusing on the individual role of having GJH and having CMP on physical functioning within patients with G-HSD/hEDS and the additional role of pain-related fear. Therefore, this study allowed to differentiate between both the physical components in tandem with pain-related fear in adolescents with G-HSD/hEDS compared to adolescents with CMP. These results suggested that once adolescents have CMP, despite being hypermobile or not, the level of pain-related fear is the contributing factor to diminished physical functioning.

The following limitations should be addressed. First, the limited number of adolescents with CMP and GJH and adolescents without CMP and GJH. Therefore, the results should be treated with caution. However, the prevalence of GJH in the general population $(18 \%)$ and GJH in the CMP population (28\%) is comparable to other studies performed in the Dutch adolescent population, despite the use of different cut-off points. ${ }^{43,44}$ Further research should be conducted in larger groups to confirm the current findings. Second, the absence of continuous physical activity measurements due to contact sports and/or swimming might result in an underestimation of the adolescent's actual PAL. Especially in the group of adolescents without CMP, who reported more non-wearing time due to different sports than the adolescents with CMP. Third, the relatively large number of analyses performed leads to an increased type I error. The final limitation is the crosssectional design of the current study, which meant no causal relationships could be confirmed. Future studies should employ longitudinal designs to examine associations 
over time in adolescents with GJH before the onset of CMP. Differences in physical and psychological functioning in adolescents who will or will not develop CMP might be important determinants for prevention of the development of CMP.

This study has clinical implications. Several studies proposed multidisciplinary rehabilitation treatment (MRT) for adolescents with G-HSD/hEDS that reduce disability. ${ }^{45-47}$ The studies of Rahman et al. ${ }^{46}$ and Bathen et al. ${ }^{45}$ included cognitivebehavioral therapy (CBT), not specifically aimed at reducing pain-related fear, alongside with physical therapy. Improvements were found in muscle strength and muscle strength endurance, a reduction of kinesiophobia, a decrease in levels of anxiety, depression, and catastrophizing thoughts, and an increase in self-efficacy. The findings of this study showed that physical functioning in hypermobile as well as non-hypermobile adolescents with CMP was diminished and that the level of pain-related fear was contributing. These results support previous findings in the literature who showed that psychological distress, such as anxiety and depression, had a strong association with disability in patients with G-HSD/hEDS. ${ }^{48}$ Therefore, it seemed to imply that in future MRT for adolescents with G-HSD/hEDS it is important to focus also on psychological components, such as pain-related fear, catastrophizing thoughts, and generalized anxiety. Furthermore, it might be advisable to include other important constructs of functional disability in adolescents with G-HSD/hEDS, such as multi-systemic dysfunction and fatigue. ${ }^{49}$

\section{Conclusions}

Adolescents with CMP had decreased muscle strength and motor performance associated with increased levels of pain-related fear compared to adolescents without CMP. Contrary to expectations, no differences were found in physical functioning and pain-related fear between adolescents with G-HSD/hEDS compared to adolescents with CMP. Furthermore, no differences in PAL was observed between adolescents with/without CMP compared to pain-free controls. These results seemed to suggest that future MRT for adolescents with G-HSD/hEDS should also focus on psychological components, such as pain-related fear and generalized anxiety. 


\section{References}

1. Merskey H, Bogduk N. Classification of Chronic Pain. 2nd ed. Seattle (WA): IASP Press; 1994.

2. Gatchel RJ, Peng YB, Peters ML, Fuchs PN, Turk DC. The biopsychosocial approach to chronic pain: scientific advances and future directions. Psychol Bull. 2007;133(4):581-624.

3. Perquin CW, Hazebroek-Kampschreur AA, Hunfeld JA, Bohnen AM, van Suijlekom-Smit LW, Passchier J, et al. Pain in children and adolescents: a common experience. Pain. 2000;87(1):51-8.

4. O'Sullivan P, Beales D, Jensen L, Murray K, Myers T. Characteristics of chronic non-specific musculoskeletal pain in children and adolescents attending a rheumatology outpatients clinic: a crosssectional study. Pediatr Rheumatol Online J. 2011;9(1):3.

5. Huguet A, Miro J. The severity of chronic pediatric pain: an epidemiological study. J Pain. 2008;9(3): 226-36.

6. Konijnenberg AY, Uiterwaal CS, Kimpen JL, van der Hoeven J, Buitelaar JK, de Graeff-Meeder ER. Children with unexplained chronic pain: substantial impairment in everyday life. Arch Dis Child. 2005;90(7):680-6.

7. Sohrbeck-Nohr O, Kristensen JH, Boyle E, Remvig L, Juul-Kristensen B. Generalized joint hypermobility in childhood is a possible risk for the development of joint pain in adolescence: a cohort study. BMC Pediatr. 2014;14:302.

8. Tobias JH, Deere K, Palmer S, Clark EM, Clinch J. Joint hypermobility is a risk factor for musculoskeletal pain during adolescence: findings of a prospective cohort study. Arthritis Rheum. 2013;65(4):1107-15.

9. Castori M, Tinkle B, Levy H, Grahame R, Malfait F, Hakim A. A framework for the classification of joint hypermobility and related conditions. Am J Med Genet C Semin Med Genet. 2017;175(1):148-57.

10. Malfait F, Francomano C, Byers P, Belmont J, Berglund B, Black J, et al. The 2017 international classification of the Ehlers-Danlos syndromes. Am J Med Genet C Semin Med Genet. 2017;175(1):8-26.

11. Remvig L, Engelbert RH, Berglund B, Bulbena A, Byers PH, Grahame R, et al. Need for a consensus on the methods by which to measure joint mobility and the definition of norms for hypermobility that reflect age, gender and ethnic-dependent variation: is revision of criteria for joint hypermobility syndrome and Ehlers-Danlos syndrome hypermobility type indicated? Rheumatology (Oxford). 2011;50(6):1169-71.

12. Fatoye F, Palmer S, Macmillan F, Rowe P, van der Linden M. Proprioception and muscle torque deficits in children with hypermobility syndrome. Rheumatology (Oxford). 2009;48(2):152-7.

13. Engelbert RH, van Bergen $M$, Henneken $T$, Helders PJ, Takken T. Exercise tolerance in children and adolescents with musculoskeletal pain in joint hypermobility and joint hypomobility syndrome. Pediatrics. 2006;118(3):690-6.

14. Schubert-Hjalmarsson E, Ohman A, Kyllerman M, Beckung E. Pain, balance, activity, and participation in children with hypermobility syndrome. Pediatr Phys Ther. 2012;24(4):339-44.

15. Celletti C, Castori M, La Torre G, Camerota F. Evaluation of kinesiophobia and its correlations with pain and fatigue in joint hypermobility syndrome/Ehlers-Danlos syndrome hypermobility type. Biomed Res Int. 2013;2013:580460.

16. Simmonds JV, Herbland A, Hakim A, Ninis N, Lever W, Aziz Q, et al. Exercise beliefs and behaviours of individuals with Joint Hypermobility syndrome/Ehlers-Danlos syndrome - hypermobility type. Disabil Rehabil. 2017:1-11.

17. Baeza-Velasco C, Bourdon C, Montalescot L, de Cazotte C, Pailhez G, Bulbena A, et al. Low- and highanxious hypermobile Ehlers-Danlos syndrome patients: comparison of psychosocial and health variables. Rheumatol Int. 2018;38(5):871-8.

18. Scheper MC, de Vries JE, Juul-Kristensen B, Nollet F, Engelbert RH. The functional consequences of generalized joint hypermobility: a cross-sectional study. BMC Musculoskeletal Disorders. 2014;15:243.

19. Scheper M, de Vries J, Beelen A, de Vos R, Nollet F, Engelbert R. Generalized joint hypermobility, muscle strength and physical function in healthy adolescents and young adults. Curr Rheumatol Rev. 2014;10(2):117-25.

20. Juul-Kristensen B, Kristensen JH, Frausing B, Jensen DV, Rogind H, Remvig L. Motor competence and physical activity in 8-year-old school children with generalized joint hypermobility. Pediatrics. 2009;124(5):1380-7. 
21. Van Meulenbroek T, Huijnen I, Stappers N, Engelbert R, Verbunt J. Generalized joint hypermobility and perceived harmfulness in healthy adolescents; impact on muscle strength, motor performance and physical activity level. Physiother Theory Pract. 2020:1-10.

22. Dekker C, Goossens ME, Bastiaenen $\mathrm{CH}$, Verbunt JA. Study protocol for a multicentre randomized controlled trial on effectiveness of an outpatient multimodal rehabilitation program for adolescents with chronic musculoskeletal pain (2B Active). BMC Musculoskelet Disord. 2016;17:317.

23. Claar RL, Walker LS. Functional assessment of pediatric pain patients: psychometric properties of the functional disability inventory. Pain. 2006;121(1-2):77-84.

24. Juul-Kristensen B, Schmedling K, Rombaut L, Lund H, Engelbert RH. Measurement properties of clinical assessment methods for classifying generalized joint hypermobility-A systematic review. Am J Med Genet C Semin Med Genet. 2017;175(1):116-47.

25. Hurley MV, Rees J, Newham DJ. Quadriceps function, proprioceptive acuity and functional performance in healthy young, middle-aged and elderly subjects. Age Ageing. 1998;27(1):55-62.

26. Drouin JM, Valovich-mcLeod TC, Shultz SJ, Gansneder BM, Perrin DH. Reliability and validity of the Biodex system 3 pro isokinetic dynamometer velocity, torque and position measurements. Eur J Appl Physiol. 2004;91(1):22-9.

27. Gustavsson A, Neeter C, Thomee P, Silbernagel KG, Augustsson J, Thomee R, et al. A test battery for evaluating hop performance in patients with an $A C L$ injury and patients who have undergone $A C L$ reconstruction. Knee Surg Sports Traumatol Arthrosc. 2006;14(8):778-88.

28. Ross MD, Langford B, Whelan PJ. Test-retest reliability of 4 single-leg horizontal hop tests. J Strength Cond Res. 2002;16(4):617-22.

29. Ottevaere C, Huybrechts I, De Meester F, De Bourdeaudhuij I, Cuenca-Garcia M, De Henauw S. The use of accelerometry in adolescents and its implementation with non-wear time activity diaries in free-living conditions. Journal of Sports Sciences. 2011;29(1):103-13.

30. Huijnen IP, Verbunt JA, Peters ML, Delespaul P, Kindermans HP, Roelofs J, et al. Do depression and pain intensity interfere with physical activity in daily life in patients with Chronic Low Back Pain? Pain. 2010;150(1):161-6.

31. Stinson JN, Kavanagh T, Yamada J, Gill N, Stevens B. Systematic review of the psychometric properties, interpretability and feasibility of self-report pain intensity measures for use in clinical trials in children and adolescents. Pain. 2006;125(1-2):143-57.

32. Verbunt JA, Nijhuis A, Vikstrom M, Stevens A, Haga N, de Jong J, et al. The psychometric characteristics of an assessment instrument for perceived harmfulness in adolescents with musculoskeletal pain (PHODA-youth). Eur J Pain. 2015;19(5):695-705.

33. Myers R. Classical and Modern Regression with Applications. 2nd ed. Belmont (MA): Duxbury Press; 1990.

34. Jones MA, Stratton $G$, Reilly $T$, Unnithan VB. Biological risk indicators for recurrent non-specific low back pain in adolescents. Br J Sports Med. 2005;39(3):137-40.

35. Sil S, Thomas S, DiCesare C, Strotman D, Ting TV, Myer G, et al. Preliminary evidence of altered biomechanics in adolescents with juvenile fibromyalgia. Arthritis Care Res (Hoboken). 2015;67(1):102-11.

36. Long AC, Palermo TM, Manees AM. Brief report: using actigraphy to compare physical activity levels in adolescents with chronic pain and healthy adolescents. J Pediatr Psychol. 2008;33(6):660-5.

37. Wilson AC, Palermo TM. Physical activity and function in adolescents with chronic pain: a controlled study using actigraphy. The Journal of Pain. 2012;13(2):121-30.

38. Stommen NC, Verbunt JA, Gorter SL, Goossens ME. Physical activity and disability among adolescents and young adults with non-specific musculoskeletal pain. Disabil Rehabil. 2012;34(17):1438-43.

39. Huijnen IPJ, Verbunt J, Wittink HM, Smeets RJEM. Physical performance measurement in chronic low back pain: measuring physical capacity of pain-related behaviour? European Journal of Physiotherapy. 2013;15(3):103-10.

40. Fisher E, Heathcote LC, Eccleston C, Simons LE, Palermo TM. Assessment of Pain Anxiety, Pain Catastrophizing, and Fear of Pain in Children and Adolescents With Chronic Pain: A Systematic Review and Meta-Analysis. J Pediatr Psychol. 2018;43(3):314-25.

41. Caes L, Fisher E, Clinch J, Tobias JH, Eccleston C. The role of pain-related anxiety in adolescents' disability and social impairment: ALSPAC data. Eur J Pain. 2015;19(6):842-51. 
42. Rombaut L, Malfait F, De Wandele I, Thijs Y, Palmans T, De Paepe A, et al. Balance, gait, falls, and fear of falling in women with the hypermobility type of Ehlers-Danlos syndrome. Arthritis Care Res (Hoboken). 2011;63(10):1432-9.

43. van Meulenbroek T, Huijnen IPJ, Wiertz CMH, Verbunt JA. Pain-Related Fear and Its Disabling Impact in Hypermobile Adolescents With Chronic Musculoskeletal Pain. J Orthop Sports Phys Ther. 2017;47(10):775-81.

44. Jelsma LD, Geuze RH, Klerks MH, Niemeijer AS, Smits-Engelsman BC. The relationship between joint mobility and motor performance in children with and without the diagnosis of developmental coordination disorder. BMC Pediatr. 2013;13:35.

45. Bathen T, Hangmann AB, Hoff M, Andersen LO, Rand-Hendriksen S. Multidisciplinary treatment of disability in ehlers-danlos syndrome hypermobility type/hypermobility syndrome: A pilot study using a combination of physical and cognitive-behavioral therapy on 12 women. Am J Med Genet A. 2013;161(12):3005-11.

46. Rahman A, Daniel C, Grahame R. Efficacy of an out-patient pain management programme for people with joint hypermobility syndrome. Clin Rheumatol. 2014;33(11):1665-9.

47. Castori M, Morlino S, Celletti C, Celli M, Morrone A, Colombi M, et al. Management of pain and fatigue in the joint hypermobility syndrome (a.k.a. Ehlers-Danlos syndrome, hypermobility type): principles and proposal for a multidisciplinary approach. Am J Med Genet A. 2012;158(8):2055-70.

48. Scheper MC, Juul-Kristensen B, Rombaut L, Rameckers EA, Verbunt J, Engelbert RH. Disability in Adolescents and Adults Diagnosed With Hypermobility-Related Disorders: A Meta-Analysis. Arch Phys Med Rehabil. 2016;97(12):2174-87.

49. Scheper MC, Nicholson LL, Adams RD, Tofts L, Pacey V. The natural history of children with joint hypermobility syndrome and Ehlers-Danlos hypermobility type: a longitudinal cohort study. Rheumatology (Oxford). 2017;56(12):2073-83. 



\section{Chapter 6}

Multidisciplinary treatment for hypermobile adolescents with chronic musculoskeletal pain

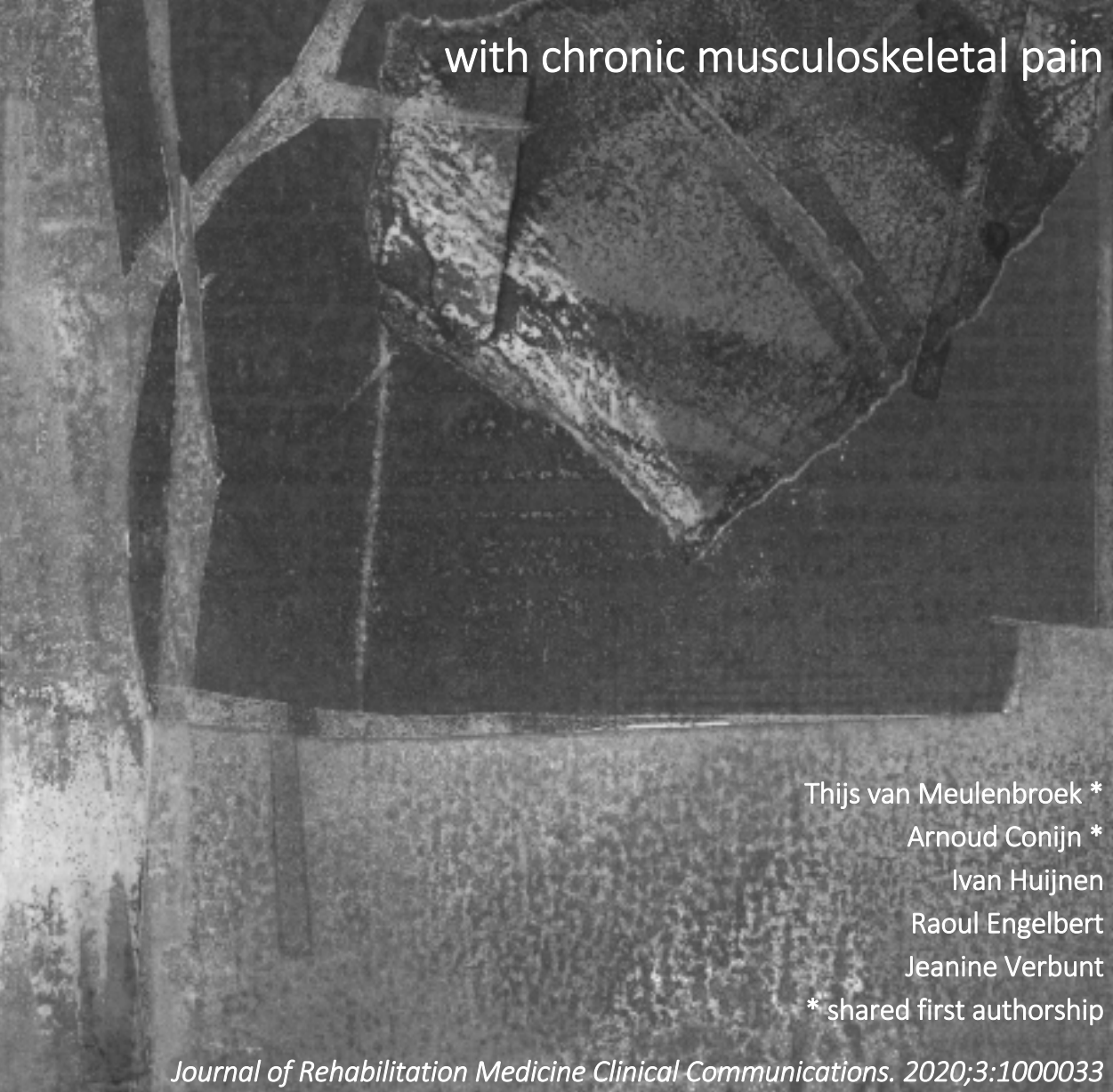




\section{Abstract}

\section{Background}

To determine whether adolescents with generalized hypermobility spectrum disorder/ hypermobile Ehlers-Danlos syndrome (G-HSD/hEDS) show changes in the level of disability, physical functioning, perceived harmfulness, and pain intensity after completing multidisciplinary rehabilitation treatment.

\section{Methods}

Pre-post-test design. Fourteen adolescents with G-HSD/hEDS participated. The multidisciplinary rehabilitation treatment consisted of a combination of physical training and exposure in vivo. Physical training aims to improve aerobic capacity, muscle strength, and propriocepsis for compensating hypermobility. Exposure in vivo aims to decrease disability and pain-related fear. Pre- and post-treatment assessments were conducted to assess the level of disability, physical functioning (motor performance, muscle strength, and physical activity level), perceived harmfulness, and pain intensity.

\section{Results}

After completing multidisciplinary rehabilitation treatment, the adolescents showed a significant and clinically relevant improvement (improvement of $67 \%, p<0.01$ ) in functional disability. Furthermore, significant improvements were found in motor performance $(p<0.01)$, muscle strength $(p<0.05)$, perceived harmfulness $(p<0.01)$, and pain intensity $(p<0.01)$ after completing multidisciplinary rehabilitation treatment.

\section{Conclusion}

Multidisciplinary rehabilitation treatment leads to a significantly and clinically relevant improvement in the level of disability for adolescents with G-HSD/hEDS. Positive effects were also found in physical functioning, perceived harmfulness, and pain intensity. Although the results of this multidisciplinary rehabilitation treatment for adolescents with G-HSD/hEDS are promising, further study is needed to confirm these findings in a randomized design. 


\section{Introduction}

Generalized joint hypermobility $(\mathrm{GJH})$ is a non-symptomatic condition characterized by increased range of motion in multiple joints due to increased laxity of connective tissue. ${ }^{1}$ Previous studies suggest a relevant relationship between chronic musculoskeletal pain (CMP) and GJH, as GJH is a risk factor for CMP. ${ }^{2,3}$ In case GJH occurs with one or more musculoskeletal manifestations, such as chronic pain, trauma, disturbed proprioception, and joint instability, it is referred to as generalized hypermobility spectrum disorder (G-HSD). ${ }^{4}$ Similar characteristics have been reported in children and adolescents with the hypermobile Ehlers-Danlos syndrome (hEDS). ${ }^{5}$ It has been suggested that G-HSD and hEDS are clinically indistinguishable $e^{4,5}$, both lacking a specific genetic profile and are therefore labelled as G-HSD/hEDS.

Compared with healthy peers, individuals with G-HSD/hEDS have a higher presence of physical complaints, such as activity-related pain ${ }^{6}$, decreased muscle strength ${ }^{7}$, impaired proprioception $^{8}$, reduced balance ${ }^{9}$, multi-systemic dysfunction (such as hyperelastic skin, orthostatic intolerance, gastrointestinal problems, and organ dysfunction) ${ }^{10}$, and significant disability. ${ }^{11}$ In addition, individuals with G-HSD/hEDS have also demonstrated an increased incidence of psychosocial complaints, such as pain-related fear ${ }^{12}$ and psychiatric conditions, including anxiety disorders, panic disorders, and depression ${ }^{13}$ compared with healthy peers.

Multidisciplinary rehabilitation treatment (MRT) in CMP may be effective in reducing disability and has been recommended in adolescents with G-HSD/hEDS. ${ }^{14,15}$ Recently, a treatment protocol for MRT for adolescents with CMP, including a programme for adolescents with G-HSD/hEDS, was published. ${ }^{16}$ Adolescents with G-HSD/hEDS received physical training sessions and exposure in vivo (EXP) sessions. Results showed a clinically relevant and statistically significant decrease in functional disability in adolescents with CMP after MRT. ${ }^{17}$ However, based on these results, no conclusions could be drawn on potential improvements specifically for the subgroup of adolescents with G-HSD/hEDS. However, the treatment and measurement protocol was implemented in usual care, which led to a higher number of adolescents with G-HSD/hEDS.

This study has 2 primary objectives: First, to determine whether adolescents with G-HSD/hEDS showed changes in the level of disability (domain of participation) after following MRT. Secondly, to study whether improvements were found in physical functioning, perceived harmfulness, and pain intensity in the adolescents with G-HSD/hEDS after MRT was finished. 


\section{Methods}

\section{Participants}

In this pre-post-test design, 14 adolescents with G-HSD/hEDS completed MRT. Inclusion criteria were: (1) indication for outpatient MRT, (2) considerable disability and fear of movement according to the expert opinion of the physician in rehabilitation medicine, (3) age 12-21 years at the start of the treatment, (4) chronic musculoskeletal complaints ( $\geq 3$ months), and (5) joint hypermobility with a Beighton score (BS) of $\geq 6$ (age of participants, $12-17$ years) or a BS of $\geq 5$ (age of participants, 18-21 years). ${ }^{5,18}$ Exclusion criteria were possible psychiatric disorders for which a psychiatric treatment is indicated and a medical (orthopaedic, rheumatic or neurological) disease that can explain the severity of pain complaints.

To describe the population at baseline, data on sociodemographic variables (age, sex, education level, duration of pain, and school absence due to pain in the last year) were collected. Furthermore, height and weight were measured without heavy clothing and shoes. Body mass index was calculated as weight in $\mathrm{kg}$ divided by the square of height in $\mathrm{m}$. Joint hypermobility was measured by a physician with a standardized protocol using the BS. The inter-rater reliability of the BS seems acceptable to be used in clinical practice. ${ }^{18}$ Psychosocial functioning was assessed with questionnaires regarding fear of pain (Fear of Pain Questionnaire-Child report; FOPQ-C), pain catastrophizing (Pain Catastrophizing Scale for Children; PCS-C), and depressive symptoms (Children's Depression Inventory; CDI). The FOPQ-C, PCS-C, and CDI have demonstrated good validity and reliability in children and adolescents. ${ }^{19-21}$

\section{Procedure}

Participants in this study were included in two different ways. First, adolescents participated in the $2 \mathrm{~B}$-Active trial. ${ }^{16}$ The purpose of this study was to evaluate whether $M R T$, including EXP, reduces functional disability in adolescents with CMP compared to care as usual. If adolescents with CMP had an indication for MRT, they were invited to participate in the study and received patient information. Adolescents younger than 18 years needed written approval from their parents and/or caregivers. The MRT for adolescents with G-HSD/hEDS consisted of a combined programme of physical training sessions and EXP sessions. A detailed description of the procedure of the trial is found elsewhere. ${ }^{16}$ Three out of nine adolescents with G-HSD/hEDS were allocated to the MRT of combined physical training sessions and EXP sessions and, in this way, they also participated in the current study. Secondly, after completion of the 2B-Active trial in $2017^{17}$, treatment and measurement protocol according to 2B-Active was implemented 
in usual care for adolescents with G-HSD/hEDS at Adelante/Maastricht University Medical Center+. In the period April 2017 to August 2019, an additional 11 adolescents with G-HSD/hEDS received MRT according to the protocol used in the 2B-Active trial. Measurements were part of the regular care. To ask adolescents and their parents and/or caregivers permission to use outcome of assessments for research purposes, they received an information letter and were asked to give informed consent. Adolescents younger than 16 years needed written approval from their parents and/or caregivers. After informed consent was given, the measurements were collected anonymously. Ethical approval for this study was granted by the Medical Ethics Committee Academic Hospital Maastricht/Maastricht University, the Netherlands (METC 2018-0520).

All adolescents participated in a pre-treatment and a post-treatment assessment. Assessments of the 3 adolescents in the 2B-Active trial were performed by an independent physiotherapist. Assessments for the additional 11 adolescents were performed by their treating physiotherapist. Finally, both data-sets were combined.

\section{Multidisciplinary rehabilitation treatment (MRT)}

During the intake the physician in rehabilitation medicine assessed the full medical history and current CMP-related complaints, disabilities, and medication used. Afterwards the physician in rehabilitation medicine decided whether the adolescents were eligible for MRT and monitored the treatment process. The MRT (total of 15 weeks) started with an intake and education session in the first week, provided by a skilled psychologist and physiotherapist. Subsequently, patients completed an 8-week physical training (2-h, 2x/week) programme led by a physiotherapist and assistant physiotherapist. Thereafter, adolescents received an additional 6 sessions (1-h, 1x/week) of EXP therapy provided by a skilled psychologist and physiotherapist. Table 6.1 provides a summary of the content of the MRT. Parallel during the MRT treatment, 3 parent meetings (in a group or individually) were offered to help parents facilitating their children with pain in learning to cope with pain. ${ }^{22}$ It was intended that both parents attend the sessions. Individual parent meetings were conducted when there were less than 3 parent couples available for the parent group. The physical training module focused on improving physical parameters, such as aerobic capacity, muscle strength, core stability, and propriocepsis, for compensating the physical impacts of GJH. The EXP module used principles of classical conditioning and cognitive-behavioural techniques to restore the normal pattern of daily functioning by reducing pain-related fear and catastrophic thinking, through exposing adolescents to fear-provoking daily life activities and movements. ${ }^{23}$ A detailed description of the MRT is found elsewhere. ${ }^{16}$ 
Table 6.1 Description of the multidisciplinary rehabilitation treatment.

\begin{tabular}{ll}
\hline Mumber of sessions & Total of 15 weeks; 40 hours; 1-h sessions. \\
Therapists & Psychologist, physiotherapist, physiotherapist assistant. \\
Treatment overview & Week 1; Session 1: Intake; cognitive analysis of pain complaints and its consequences \\
& and identifying movements/activities that are threatening using the Photograph \\
& Series of Daily Activities Youth (PHODA-youth). \\
& Week 1; Session 2: Education; explaining the treatment rationale and completing a \\
& personal fear-avoidance model. \\
& Week 2-9; Session 3-34: Physical training (combination of hydrotherapy and fitness) \\
& focusing on aerobic capacity, muscle strength, core stability, and propriocepsis. \\
& Adolescents also received home exercises. \\
& Week 10-15; Session 35-40: Exposure with behavioural experiments, fear provoking \\
& activities and movements, generalization, and relapse prevention. \\
\hline
\end{tabular}

Abbreviations: MRT = multidisciplinary rehabilitation treatment; G-HSD = generalized hypermobility spectrum disorder; hEDS = hypermobile Ehlers-Danlos syndrome.

\section{Primary outcome measure}

The primary outcome was the Functional Disability Inventory (FDI). The FDI is a selfreport measurement instrument for adolescents, which is used for measuring perceived difficulty in performing activities at school, at home, and in recreational or social interactions. The instrument consists of 15 items rated on a 5-point scale $(0=$ no trouble to 4=impossible). The FDI total score ranges from 0 to 60 , with a higher score demonstrating greater disability. Scores ranging from 0 to 12 are classified as none or minimal disability, 13 to 29 as moderate disability, and scores $\geq 30$ reflect severe disability. ${ }^{24}$ The FDI is a reliable and valid instrument to evaluate pain-related disability in adolescents. ${ }^{25}$

\section{Secondary outcome measures}

\section{Physical functioning}

The level of physical functioning was determined by measuring motor performance, muscle strength, muscle strength endurance, and physical activity level.

For measuring motor performance, the single leg hop for distance (SLHD) was used. The adolescents were asked to jump as far as possible on a single leg, without losing balance. The distance was measured in centimetres from the toe at push-off to the heel at the place the adolescents landed. After one practice attempt, the greatest distance measurement of 3 valid hops with both sides was used for further analyses. The SLHD has demonstrated high intra-subject reliability. ${ }^{26}$

To measure isokinetic muscle strength in knee extensors and flexors, the Biodex System 3 Pro dynamometer (Biodex Medical Systems, Shirley, NY, USA) was used. The adolescent was invited to take place in an upright sitting position and the tested leg was 
stabilized with a fixation strap. The lever arm was attached to the adolescent's lower leg by a padded cuff $2 \mathrm{~cm}$ proximal to the medial malleolus, and the axis of movement of the dynamometer was in line with the axis of movement of the knee flexion/extension. After one try-out attempt, 5 duplications of maximal voluntary concentric knee flexion and extension were measured at the angular velocity of $60 \%$ second. Peak torque (PT, $\mathrm{Nm}$ ) was assessed, and represents for the highest muscular force output similar to one repetition maximum effort in isotonic, and represents the muscle's maximum strength capability. PT/body weight is the PT normalized for body weight, used to standardize and compare scores in further analyses.

The same set-up as above (isokinetic muscle strength, Biodex System 3 Pro dynamometer) was used to measure isokinetic muscle strength endurance. After one try-out attempt, the adolescent had to perform 30 repetitions of maximal concentric knee flexion and extension with an angular velocity of $240 \%$ ssecond. The test was carried out $60 \mathrm{~s}$ after the isokinetic test of $60^{\circ} /$ second. Total work $(\mathrm{J})$ is the work produced throughout the test and represents the muscle's capability. The Biodex isokinetic dynamometer has shown to be a reliable and valid measure. ${ }^{27}$

The level of physical activity (PAL) during daily life was measured using a tri-axial accelerometer (AX3, Axivity, Newcastle, UK) attached to the waist using plaster (Tegaderm Film, $10 \times 12 \mathrm{~cm}$ ). The adolescents were instructed to remove the accelerometer during activities potential harmful for the device, such as contacts sports or swimming. In addition, all adolescents were asked to keep a diary of data interruption, such as removing the device and the reason for doing this. Furthermore, wake-up time and sleeping were noted. To be included as a valid measurement, a minimum of 3 weekdays with at least 10 hours of recording and one weekend day with at least 8 hours of recording had to be available during the 7-day monitoring period. ${ }^{28}$ Raw data processing was performed using Matlab (The Math Works Inc., Natick, MA, USA). An algorithm, based on the method which was used for the Actiwatch 7 (Philips, Eindhoven, the Netherlands), was designed to obtain activity counts from the raw data, resulting in one resultant acceleration signal. This signal was rectified and filtered using a high-pass filter at $3 \mathrm{~Hz}$ and a low-pass filter at $10 \mathrm{~Hz}$. The highest recorded sample per second was selected and summed per minute (counts/minute). Daily uptime was defined as the period between getting up and going to sleep (min).

PAL was expressed as 3 quantities:

1. Total activity (TA) during uptime, determined by the total sum of counts during uptime.

2. Mean activity (MA) level during $24 \mathrm{~h}$, which refers to the mean number of counts per min per day. Sleeping time is included in the total score. 
3. Peak activity (PA) level, calculated as the highest number of counts achieved in 1 min per daytime wake period.

TA and PA measurements were calculated as a total and separately for the week and weekend days.

\section{Perceived harmfulness (PHODA-youth)}

The Photograph Series of Daily Activities for youth (PHODA-youth) assessed perceived harmfulness. This consists of 51 age-specific photographs that had to be rated on a scale 0-10 ( $0=$ not harmful at all to 10=extremely harmful). The PHODA-youth consists of 3 subscales: activities of daily living and household (PHODA-ADL; 13 items), intensive physical activities (PHODA-PA; 27 items), and social activities (PHODA-SA; 11 items). The total score was calculated by summing all items (range 0-510) and subscale scores were calculated by summing the scores on all items in that subscale. Higher scores indicate higher levels of perceived harmfulness. The PHODA-youth has demonstrated to be valid and reliable in adolescents with CMP. ${ }^{29}$

\section{Pain intensity (VAS)}

The visual analogue scale (VAS) consisted of a pre-measured line (100-mm) that ranges across a continuum from "no pain" to "worst pain imaginable". The adolescents were asked to score 3 VAS scales: (1) current pain, (2) the worst/most severe pain experienced in the last week, and (3) the least pain experienced in the last week. The mean of these 3 VAS scores was calculated and used to express pain intensity in the analyses. The VAS demonstrated good reliability and validity in measuring pain intensity in children older than 8 years. ${ }^{30}$

\section{Statistical analysis}

Data analysis was performed using the IBM Statistical Package for the Social Sciences (SPSS), version 25.0 (IBM Corp, Armonk, NY, USA). Due to the small sample size, normality of variables cannot be assumed and therefore non-parametric tests were used. ${ }^{31}$ Descriptive data of sociodemographic variables, anthropometric measurements, and hypermobility were presented as median and interquartile range (IQR). Statistically significant inter-individual changes for functional disability, physical functioning, perceived harmfulness, and pain intensity were assessed using the Wilcoxon signed-rank test. Test statistics were presented by the corresponding $z$, the significance value $(p)$, and an effect size $(r)$. Effect sizes were calculated by dividing the $z$-score through the root of the number of observations. The effect size $r$ was described indicating small effects from $r \geq 0.1$ to $r<0.3$, medium effects from $r \geq 0.3$ to $r<0.5$, and large effects from $r \geq 0.5$. P-values less than 0.05 were considered statistically significant. 


\section{Results}

\section{Descriptive analyses}

Fourteen Caucasian adolescents (13 females, 1 male) participated in this study with a median age of 17.5 years (IQR 16.0-20.3). The median BS was 6 (IQR 6-7). At baseline, the median level of the FOPQ-C was 50 (IQR 34-61), which almost represents a high level of pain-related fear according to the norm (score $\geq 51$ ). ${ }^{19}$ The median level of the PCSC-C was 26 (IQR 17-34), meaning high levels of catastrophizing thoughts about pain according to the norm (high $\geq 26$ ). ${ }^{32}$ For depressive symptoms, the median level of the CDI was 19 (IQR 13-22), which indicates the adolescents were at risk for depression (cutoff point $\geq 16) .{ }^{21}$ Baseline sociodemographic and anthropometric measurements were presented in Table 6.2. One adolescent did not complete both PHODA-youth measurements and the post-treatment FDI. Four adolescents did not complete the PAL measurements due to allergic reactions from plaster $(n=2)$ and unwilling to wear the accelerometer $(n=2)$ and one of them also did not complete the post-treatment PHODAyouth. $A 6^{\text {th }}$ adolescent did not meet the criteria for a valid post-treatment PAL registration; instead, a minimum of 3 weekdays with at least 10 hours of recording only one valid weekday was measured.

\section{Outcomes of multidisciplinary rehabilitation treatment}

Compared with the pre-treatment level of disability (median 24) adolescents with G-HSD/hEDS showed significant improvements at post-treatment (median 8), $z=-3.18$, $p<0.01, r=-0.61$. The individual scores of the FDI are presented in Figure 6.1.

Regarding secondary outcomes, significant improvements were found in post-treatment motor performance $(z=-3.30, p<0.01, r=-0.62)$, knee extension muscle strength $(z=-3.30$, $p<0.01, r=-0.62)$, knee flexion muscle strength $(z=-3.30, p<0.01, r=-0.62)$, knee extension muscle strength endurance $(z=-2.35, p<0.05, r=-0.42)$, and knee flexion muscle strength endurance $(z=-2.35, p<0.05, r=-0.42)$ compared with pre-treatment. Analyses of the nonpreferred leg were not presented, but results were comparable to outcomes, as presented of the preferred leg. There were no statistical differences found in all posttreatment PAL conditions compared with pre-treatment PAL conditions. Regarding perceived harmfulness, post-treatment significant improvements were found in the total score $(z=-3.06, p<0.01, r=-0.61)$ and all subscales (PHODA-ADL: $z=-2.67, p<0.01, r=-0.52$; PHODA-PA: $z=-3.06, p<0.01, r=-0.61$; PHODA-SA: $z=-2.80, p<0.01, r=-0.54)$. Furthermore, post-treatment significant improvements in self-reported pain intensity were found $(z=-2.79, p<0.01, r=-0.51)$ (Table 6.3). 
Table 6.2 Baseline sociodemographic and clinical characteristics of the study sample $(n=14)$.

\begin{tabular}{|c|c|}
\hline Characteristics & \\
\hline Age, years, median (IQR) & $17.5(16.0-20.3)$ \\
\hline Females/males, $\mathrm{n}$ & $13 / 1$ \\
\hline Hypermobility (BS), median (IQR) & $6(6-7)$ \\
\hline Weight, kg, median (IQR) & $70.7(53.8-78.0)$ \\
\hline Height, m, median (IQR) & $1.68(1.65-1.76)$ \\
\hline $\mathrm{BMI}, \mathrm{kg} / \mathrm{m}^{2}$, median (IQR) & $23.8(18.6-27.3)$ \\
\hline \multicolumn{2}{|l|}{ Education level, n } \\
\hline Secondary education & 7 \\
\hline Tertiary education & 7 \\
\hline \multicolumn{2}{|l|}{ Duration of pain, $n$} \\
\hline 3-6 months & 1 \\
\hline 6-12 months & 2 \\
\hline $1-5$ years & 9 \\
\hline$>5$ years & 2 \\
\hline \multicolumn{2}{|l|}{ Absence of school, $n$} \\
\hline 0-14 days & 7 \\
\hline $15-30$ days & 2 \\
\hline 1-3 months & 3 \\
\hline 4-6 months & 1 \\
\hline 7-12 months & 1 \\
\hline FOPQ-C, median (IQR) & $50(34-61)$ \\
\hline PCS-C, median (IQR) & $26(17-34)$ \\
\hline $\mathrm{CDI}^{\mathrm{a}}$, median $(\mathrm{IQR})$ & $19(13-22)$ \\
\hline
\end{tabular}

Abbreviations: $I Q R=$ interquartile range; $B S=$ Beighton score; $B M I=$ body mass index; $F O P Q-C=$ Fear of Pain Questionnaire-Child report; PSC-C = Pain Catastrophizing Scale for Children; $C D I=$ Children's Depression Inventory. ${ }^{a} \mathrm{n}=13$.

Individual FDI score (pre-post treatment)

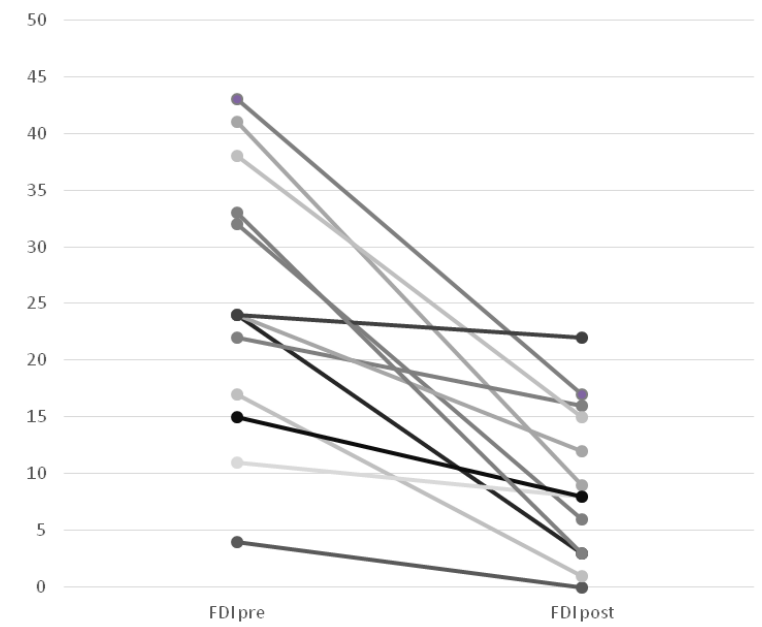

Figure 6.1 Reduction in individual Functional Disability Inventory (FDI) scores of the adolescents with generalized hypermobility spectrum disorder/hypermobile Ehlers-Danlos syndrome (G-HSD/hEDS) ( $n=13)$. 
Table 6.3 Results of the primary and secondary outcomes for all time-points of measurements.

\begin{tabular}{lccccc}
\hline MRT (n=14) & Pre-treatment & & \multicolumn{2}{c}{ Test statistics } \\
& median (range) & $\begin{array}{c}\text { Difference between post- and } \\
\text { pre-treatment (median; IQR) }\end{array}$ & $z$ & $p$ & $r$ \\
\hline Functional disability & & & & & \\
FDI & $24.0(16.5-34.3)$ & $-16.0(-26.0--5.0)^{\mathrm{a}}$ & -3.18 & 0.001 & -0.61 \\
Physical functioning & & & & & \\
SLHD pref & $99.5(93.0-132.3)$ & $20.0(11.8-30.0)$ & -3.30 & 0.001 & -0.62 \\
SLHD non-pref & $111.0(92.8-129.0)$ & $17.0(7.5-25.3)$ & -3.30 & 0.001 & -0.62 \\
PT/BW ext pref & $104.8(55.0-153.8)$ & $67.3(46.5-85.3)$ & -3.30 & 0.001 & -0.62 \\
PT/BW ext non-pref & $94.9(73.3-130.5)$ & $44.3(22.0-78.9)$ & -3.30 & 0.001 & -0.62 \\
PT/BW flex pref & $77.1(35.7-90.2)$ & $36.9(23.4-52.7)$ & -3.30 & 0.001 & -0.62 \\
PT/BW flex non-pref & $69.7(61.4-87.0)$ & $26.1(22.1-38.2)$ & -3.23 & 0.001 & -0.61 \\
TW ext pref & $531.7(326.2-928.4)$ & $567.7(241.9-859.5)$ & -2.35 & 0.019 & -0.42 \\
TW ext non-pref & $689.3(282.3-1286.9)$ & $646.8(423.3-910.2)$ & -2.42 & 0.016 & -0.43 \\
TW flex pref & $730.1(340.2-1201.9)$ & $418.9(185.3-667.3)$ & -2.35 & 0.019 & -0.42 \\
TW flex non-pref & $682.4(310.9-1213.9)$ & $423.2(297.3-763.1)$ & -2.48 & 0.013 & -0.45 \\
Total activity & $1.4 \times 10^{5}\left(1.2 \times 10^{5}-1.8 \times 10^{5}\right)^{b}$ & $-0.9 \times 10^{4}\left(-3.9 \times 10^{5}-3.6 \times 10^{5}\right)^{\mathrm{c}}$ & -0.18 & 0.859 & -0.04 \\
Mean activity & $98.1(81.4-124.5)^{\mathrm{b}}$ & $-6.9(-27.1-25.0)^{\mathrm{c}}$ & -0.18 & 0.859 & -0.04 \\
Peak activity & $1241.8(905.0-1573.1)^{\mathrm{b}}$ & $65.1(-157.4-338.5)^{\mathrm{c}}$ & -0.65 & 0.515 & -0.15 \\
Perceived harmfulness & & & & & \\
PHODA ADL & $16.0(4.9-39.0)^{\mathrm{a}}$ & $-13.0(-35.0--2.6)^{\mathrm{d}}$ & -2.67 & 0.008 & -0.52 \\
PHODA PA & $147.0(75.5-191.5)^{\mathrm{a}}$ & $-116.0(-154.3--43.7)^{\mathrm{d}}$ & -3.06 & 0.002 & -0.61 \\
PHODA SA & $35.0(9.0-45.5)^{\mathrm{a}}$ & $-21.8(-35.9--2.8)^{\mathrm{d}}$ & -2.80 & 0.005 & -0.54 \\
PHODA total & $189.0(100.0-273.3)^{\mathrm{a}}$ & $-151.0(-229.3--47.7)^{\mathrm{d}}$ & -3.06 & 0.002 & -0.61 \\
Pain intensity & & & & & \\
VAS & $41.3(31.6-58.2)$ & $-26.0(-45.6--5.4)$ & -2.79 & 0.005 & -0.51 \\
\hline
\end{tabular}

Abbreviations: pref = preferred leg; non-pref = non-preferred leg; ext = extension; flex = flexion; FDI = Functional Disability Inventory; SLHD = single leg hop for distance; PT/BW = peak torque normalized for body weight; TW = total work; PHODA = Photograph Series of Daily Activities; PHODA ADL = subscore daily life activities; PHODA PA = subscore physical activities; PHODA SA = subscore social activities; VAS = visual analogue scale; MRT = multidisciplinary rehabilitation treatment. ${ }^{a} n=13 .{ }^{b} n=10 .{ }^{c} n=9 .{ }^{d} n=12$.

\section{Discussion}

This study demonstrated that adolescents with G-HSD/hEDS show significant improvements in functional disability following MRT. These improvements were represented as a large effect size $(r>0.5)$. According to the norm of the FDI, the pretreatment level of 24 points out of 60 is classified as moderate disability (score 13-29), while the post-treatment median level of 8 points out of 60 represents no/minimal disability (score 0-12). ${ }^{24}$ Thus, after MRT adolescents with G-HSD/hEDS were able to function quite well. The decrease in functional disability (16 points) can be seen as a clinically relevant change (FDI reduction of $\geq 7.8$ points). ${ }^{33}$

Furthermore, MRT showed improvements in pain intensity. The reduction in pain (63\%) is remarkable, since MRT focused on reducing the level of disability and pain reduction 
was not the main goal. This finding could be attributed to improvements in propriocepsis and deconditioning after completing the physical training sessions. ${ }^{8}$ The results showed a $63 \%$ reduction in pain intensity, which is considered as a clinically significant improvement for average pain intensity. ${ }^{34}$

In addition, MRT showed post-treatment improvements in most subcategories of physical functioning, such as muscle strength, muscle strength endurance, and motor performance which is determined by coordination, balance, and joint stability. These improvements in the physical parameters are hypothesized to be important for compensating the physical impacts of GJH. In contrast to the improvements in the level of functioning, the level of physical activity in daily life remains similar. A possible explanation for this finding could be that adolescents with G-HSD/hEDS perceive their daily life activities as improved, which is reflected by a decrease in the perceived difficulty of performing activities at school, at home, and in recreational or social interactions. However, the objective measurements presented as total counts did not confirm these beliefs. Finally, MRT showed improvements in pain-related fear, measured as perceived harmfulness. This reduction might be due to the fact that pain and fear share some common brain networks and therefore a decrease in pain intensity might also lead to a decrease of pain-related fear. ${ }^{35}$ The decrease in pain-related fear might also be explained due to clarification of the condition and exposing adolescents to fearprovoking daily life activities and movements.

It seems remarkable that despite the small sample size of this study, significant improvements were found in most of the outcome variables with medium-to-large effects. Earlier studies in children and adults with G-HSD/hEDS, who received physical training only, showed comparable results regarding improvements in pain intensity. ${ }^{36,37}$ In addition, a RCT study showed that a physiotherapist-prescribed exercise programme for children with G-HSD/hEDS and hypermobile knees led to improvements in knee muscle strength, pain reduction, and psychosocial factors. ${ }^{38}$ However, our population had considerable disability and fear of movement, and therefore MRT was indicated. Furthermore, the current findings regarding pain intensity and physical functioning were also in line with results of children aged 5-16 years with G-HSD/hEDS receiving a multidisciplinary intervention with both physiotherapy and occupational therapy. ${ }^{39}$ However, no specific attention towards psychological symptoms, such as anxiety, was conducted, the main outcome measure was child-reported pain and no specific parent guidance was part of the intervention. Another study with combined physical and cognitive-behavioural therapy showed comparable results regarding improvements in performance of daily life activities, increased muscle strength and endurance, and reduced kinesiophobia. ${ }^{40}$ However, the participants in the study of Bathen et al. ${ }^{40}$ were exclusively female adults, were hospitalized for 2.5 weeks as part of the treatment, and 
the cognitive-behavioural approach aimed at raising awareness of thought and patterns in life. To our knowledge, our study is the first interventional study focusing on outpatient rehabilitation treatment for adolescents with G-HSD/hEDS to investigate the outcome of MRT, containing a combination of physical training and EXP, on the level of disability, physical functioning, pain-related fear, and pain intensity.

This study has some limitations, which should be considered. The first limitation of this study is the pre-post-test design with the lack of a control group. Overall, this limits our ability to infer causation of any improvements observed to participation in MRT. Nonspecific factors, such as expectancy, also cannot be controlled in a single-group study design and may play an important role in the beneficial results found in this study. A second limitation is the small sample size in this study. Although we intended to perform a subgroup analysis, the treatment effect in a subgroup of adolescents with G-HSD/hEDS in the 2B-Active trial, the number of patients needed was not attained. In the current study, we are able to present information on the progress of adolescents with G-HSD/hEDS following MRT. A third limitation is the pre-post-treatment assessment by the treating physiotherapist during the usual care, which could be prone to bias. To limit the bias, a measurement protocol was used with prescribed steps to ensure the same procedure and order of measurements, which was used in the 2B-Active trial. The fourth limitation is the relatively large number of analyses performed. Therefore, there is an elevated risk of a type I error. However, 16 out of the 19 analyses were considered significant different, and even lowering $\alpha$ still leads to mainly significant outcomes.

The results of this study are promising and could have clinical implications. Many authors propose MRT for adolescents with G-HSD/hEDS. ${ }^{14,15,39,40}$ In consideration of the multidimensional and diversity of symptoms and conditions the current study suggests that a multidisciplinary approach is recommended. However, our findings are based on a small sample size without a randomized controlled group and, as a result, this interpretation should be treated with caution. Therefore, further studies are needed using a randomized design with larger sample sizes, including a long-term follow-up evaluation to investigate the effectiveness of MRT in adolescents with G-HSD/hEDS. Furthermore, in this study we did not include measures of multi-systemic dysfunction and fatigue, which are important constructs of functional disability. ${ }^{10}$ In particular, multisystemic dysfunction was related to a deteriorating level of functioning. Therefore, it might be advisable to include these constructs in further studies and clinical practice to identify adolescents with G-HSD/hEDS who are at risk of further decline in functioning. 
Chapter 6

\section{Conclusion}

In adolescents with G-HSD/hEDS, MRT leads to a significantly and clinically relevant improvement in functional disability. Furthermore, improvements in physical functioning, perceived harmfulness, and pain intensity occurred after MRT. Although the results of this MRT in rehabilitation care for adolescents with G-HSD/hEDS are promising, these findings should be replicated in a study with a randomized design. 


\section{References}

1. Juul-Kristensen B, Rogind H, Jensen DV, Remvig L. Inter-examiner reproducibility of tests and criteria for generalized joint hypermobility and benign joint hypermobility syndrome. Rheumatology (Oxford). 2007;46(12):1835-41.

2. Tobias JH, Deere K, Palmer S, Clark EM, Clinch J. Joint hypermobility is a risk factor for musculoskeletal pain during adolescence: findings of a prospective cohort study. Arthritis Rheum. 2013;65(4):1107-15.

3. Sohrbeck-Nohr O, Kristensen JH, Boyle E, Remvig L, Juul-Kristensen B. Generalized joint hypermobility in childhood is a possible risk for the development of joint pain in adolescence: a cohort study. BMC Pediatr. 2014;14:302.

4. Castori M, Tinkle B, Levy H, Grahame R, Malfait F, Hakim A. A framework for the classification of joint hypermobility and related conditions. Am J Med Genet C Semin Med Genet. 2017;175(1):148-57.

5. Malfait F, Francomano C, Byers P, Belmont J, Berglund B, Black J, et al. The 2017 international classification of the Ehlers-Danlos syndromes. Am J Med Genet C Semin Med Genet. 2017;175(1):8-26.

6. Adib N, Davies K, Grahame R, Woo P, Murray KJ. Joint hypermobility syndrome in childhood. A not so benign multisystem disorder? Rheumatology (Oxford). 2005;44(6):744-50.

7. Engelbert RH, van Bergen M, Henneken T, Helders PJ, Takken T. Exercise tolerance in children and adolescents with musculoskeletal pain in joint hypermobility and joint hypomobility syndrome. Pediatrics. 2006;118(3):e690-6.

8. Sahin N, Baskent A, Cakmak A, Salli A, Ugurlu H, Berker E. Evaluation of knee proprioception and effects of proprioception exercise in patients with benign joint hypermobility syndrome. Rheumatol Int. 2008;28(10):995-1000.

9. Schubert-Hjalmarsson E, Ohman A, Kyllerman M, Beckung E. Pain, balance, activity, and participation in children with hypermobility syndrome. Pediatr Phys Ther. 2012;24(4):339-44.

10. Scheper MC, Nicholson LL, Adams RD, Tofts L, Pacey V. The natural history of children with joint hypermobility syndrome and Ehlers-Danlos hypermobility type: a longitudinal cohort study. Rheumatology (Oxford). 2017;56(12):2073-83.

11. Scheper MC, Juul-Kristensen B, Rombaut L, Rameckers EA, Verbunt J, Engelbert RH. Disability in Adolescents and Adults Diagnosed With Hypermobility-Related Disorders: A Meta-Analysis. Arch Phys Med Rehabil. 2016;97(12):2174-87.

12. Celletti C, Castori M, La Torre G, Camerota F. Evaluation of kinesiophobia and its correlations with pain and fatigue in joint hypermobility syndrome/Ehlers-Danlos syndrome hypermobility type. Biomed Res Int. 2013;2013:580460.

13. Smith TO, Easton V, Bacon H, Jerman E, Armon K, Poland F, et al. The relationship between benign joint hypermobility syndrome and psychological distress: a systematic review and meta-analysis. Rheumatology (Oxford). 2014;53(1):114-22.

14. Scheper MC, Engelbert RH, Rameckers EA, Verbunt J, Remvig L, Juul-Kristensen B. Children with generalised joint hypermobility and musculoskeletal complaints: state of the art on diagnostics, clinical characteristics, and treatment. Biomed Res Int. 2013;2013:121054.

15. Castori M, Morlino S, Celletti C, Celli M, Morrone A, Colombi M, et al. Management of pain and fatigue in the joint hypermobility syndrome (a.k.a. Ehlers-Danlos syndrome, hypermobility type): principles and proposal for a multidisciplinary approach. Am J Med Genet A. 2012;158A(8):2055-70.

16. Dekker C, Goossens ME, Bastiaenen $\mathrm{CH}$, Verbunt JA. Study protocol for a multicentre randomized controlled trial on effectiveness of an outpatient multimodal rehabilitation program for adolescents with chronic musculoskeletal pain (2B Active). BMC Musculoskelet Disord. 2016;17:317.

17. van den Bogert - Dekker C. Adolescent Chronic Pain. Rehabilitation treatment targeting pain-related fear [dissertation]. Heerlen (NL): Maastricht University; 2018.

18. Juul-Kristensen B, Schmedling K, Rombaut L, Lund H, Engelbert RH. Measurement properties of clinical assessment methods for classifying generalized joint hypermobility-A systematic review. Am J Med Genet C Semin Med Genet. 2017;175(1):116-47.

19. Simons LE, Sieberg CB, Carpino E, Logan D, Berde C. The Fear of Pain Questionnaire (FOPQ): assessment of pain-related fear among children and adolescents with chronic pain. J Pain. 2011;12(6):677-86. 
20. Crombez G, Bijttebier P, Eccleston C, Mascagni T, Mertens G, Goubert L, et al. The child version of the pain catastrophizing scale (PCS-C): a preliminary validation. Pain. 2003;104(3):639-46.

21. Roelofs J, Braet C, Rood L, Timbremont B, van Vlierberghe L, Goossens L, et al. Norms and screening utility of the Dutch version of the Children's Depression Inventory in clinical and nonclinical youths. Psychol Assess. 2010;22(4):866-77.

22. Wiertz C, Goossens M, Spek EM, Verbunt JA. A cognitive-behavioral program for parents of children with chronic musculoskeletal pain; A feasibility study. Eur J Pain. 2017;21(9):1571-81.

23. den Hollander M, Heijnders N, de Jong JR, Vlaeyen JWS, Smeets R, Goossens M. Exposure in Vivo Versus Pain-Contingent Physical Therapy in Complex Regional Pain Syndrome Type I: A Cost-Effectiveness Analysis. Int J Technol Assess Health Care. 2018;34(4):400-9.

24. Kashikar-Zuck S, Flowers SR, Claar RL, Guite JW, Logan DE, Lynch-Jordan AM, et al. Clinical utility and validity of the Functional Disability Inventory among a multicenter sample of youth with chronic pain. Pain. 2011;152(7):1600-7.

25. Claar RL, Walker LS. Functional assessment of pediatric pain patients: psychometric properties of the functional disability inventory. Pain. 2006;121(1-2):77-84.

26. Ross MD, Langford B, Whelan PJ. Test-retest reliability of 4 single-leg horizontal hop tests. J Strength Cond Res. 2002;16(4):617-22.

27. Drouin JM, Valovich-mcLeod TC, Shultz SJ, Gansneder BM, Perrin DH. Reliability and validity of the Biodex system 3 pro isokinetic dynamometer velocity, torque and position measurements. Eur J Appl Physiol. 2004;91(1):22-9.

28. Trost SG, Mclver KL, Pate RR. Conducting accelerometer-based activity assessments in field-based research. Med Sci Sports Exerc. 2005;37(11 Suppl):S531-43.

29. Verbunt JA, Nijhuis A, Vikstrom M, Stevens A, Haga N, de Jong J, et al. The psychometric characteristics of an assessment instrument for perceived harmfulness in adolescents with musculoskeletal pain (PHODAyouth). Eur J Pain. 2015;19(5):695-705.

30. Stinson JN, Kavanagh T, Yamada J, Gill N, Stevens B. Systematic review of the psychometric properties, interpretability and feasibility of self-report pain intensity measures for use in clinical trials in children and adolescents. Pain. 2006;125(1-2):143-57.

31. Nahm FS. Nonparametric statistical tests for the continuous data: the basic concept and the practical use. Korean J Anesthesiol. 2016;69(1):8-14.

32. Pielech M, Ryan M, Logan D, Kaczynski K, White MT, Simons LE. Pain catastrophizing in children with chronic pain and their parents: proposed clinical reference points and reexamination of the Pain Catastrophizing Scale measure. Pain. 2014;155(11):2360-7.

33. Sil S, Arnold LM, Lynch-Jordan A, Ting TV, Peugh J, Cunningham N, et al. Identifying treatment responders and predictors of improvement after cognitive-behavioral therapy for juvenile fibromyalgia. Pain. 2014;155(7):1206-12.

34. Mease PJ, Spaeth M, Clauw DJ, Arnold LM, Bradley LA, Russell IJ, et al. Estimation of minimum clinically important difference for pain in fibromyalgia. Arthritis Care Res (Hoboken). 2011;63(6):821-6.

35. Vogt BA. Pain and emotion interactions in subregions of the cingulate gyrus. Nat Rev Neurosci. 2005;6(7):533-44.

36. Daman M, Shiravani F, Hemmati L, Taghizadeh S. The effect of combined exercise therapy on knee proprioception, pain intensity and quality of life in patients with hypermobility syndrome: A randomized clinical trial. J Bodyw Mov Ther. 2019;23(1):202-5.

37. Kemp S, Roberts I, Gamble C, Wilkinson S, Davidson JE, Baildam EM, et al. A randomized comparative trial of generalized vs targeted physiotherapy in the management of childhood hypermobility. Rheumatology (Oxford). 2010;49(2):315-25.

38. Pacey V, Tofts L, Adams RD, Munns CF, Nicholson LL. Exercise in children with joint hypermobility syndrome and knee pain: a randomised controlled trial comparing exercise into hypermobile versus neutral knee extension. Pediatr Rheumatol Online J. 2013;11(1):30.

39. Bale $P$, Easton V, Bacon $H$, Jerman E, Watts L, Barton $G$, et al. The effectiveness of a multidisciplinary intervention strategy for the treatment of symptomatic joint hypermobility in childhood: a randomised, single Centre parallel group trial (The Bendy Study). Pediatr Rheumatol Online J. 2019;17(1):2. 
Multidisciplinary treatment for hypermobile adolescents with chronic musculoskeletal pain

40. Bathen T, Hangmann AB, Hoff M, Andersen LO, Rand-Hendriksen S. Multidisciplinary treatment of disability in ehlers-danlos syndrome hypermobility type/hypermobility syndrome: A pilot study using a combination of physical and cognitive-behavioral therapy on 12 women. Am J Med Genet A. 2013;161A(12):3005-11. 



\section{Chapter

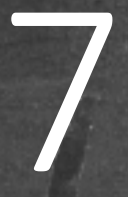

General discussion 



\section{General discussion}

Chronic musculoskeletal pain (CMP) in adolescents may have a negative impact on physical, psychological, and social functioning. ${ }^{1-3}$ Two important factors to understand the complexity of musculoskeletal pain and explain the disabling impact of CMP are the presence of pain-related fear ${ }^{4,5}$ and generalized joint hypermobility (GJH). ${ }^{6,7}$ In this dissertation, the influence of both pain-related fear and GJH on physical functioning in adolescents (12-21 years) with CMP was evaluated. Knowledge of these potential factors may enable to further disentangle the complexity of disability in adolescents with CMP. Better understanding influences the development of tailored and personalised treatments for hypermobile adolescents with CMP.

For many individuals, GJH can be an advantage, such as in the performance of sports and arts. ${ }^{8,9}$ However, GJH and CMP are also features of generalized hypermobility spectrum disorder (G-HSD) and hypermobile Ehlers-Danlos syndrome (hEDS) in addition to a wide range of other symptoms affecting musculoskeletal, gastrointestinal, cardiovascular, and autonomic nervous symptoms. ${ }^{10}$ The terms G-HSD/hEDS replaced the old nomenclature of joint hypermobility syndrome (JHS) and Ehlers-Danlos syndrome hypermobility type (EDS-HT) according to recently established unified criteria. ${ }^{11,12}$ Within this dissertation we referred to G-HSD/hEDS as a general denominator of GJH-related disorders with CMP to cover the new and historical diagnoses. Therefore, it should be noted that the consequences of these new unified criteria for patient demographics and disease characteristics are unknown yet.

In this general discussion, first the main results presented in this dissertation are summarized and discussed. Then, the implications for clinical practice, methodological considerations, and recommendations for future research are discussed. Finally, conclusions are drawn.

\section{Main findings}

In the topical review presented in chapter 2 we hypothesized that GJH and pain-related fear are both important factors in the development and maintenance of CMP eventually leading to disabling pain in adolescents with G-HSD/hEDS. The fear-avoidance model (FAM) explains the disabling role of pain-related fear in adolescents and adults with CMP. ${ }^{13,14}$ We proposed an applied FAM to explain the hypothesized interaction between GJH and pain-related fear (Figure 7.1). 


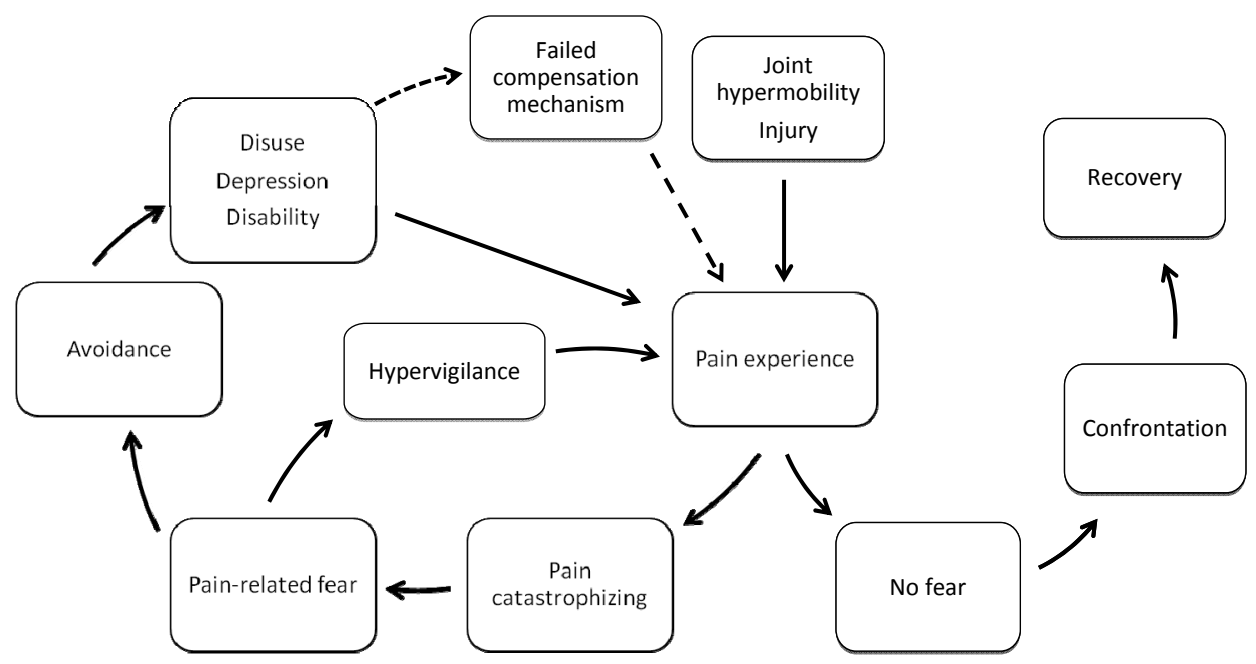

Figure 7.1 The applied fear-avoidance model for adolescents with G-HSD/hEDS.

GJH is hypothesized to make adolescents more vulnerable for developing CMP. This is due to an increased risk of (re)injury and experiencing more frequent musculoskeletal pain, eventually leading towards the diagnosis of G-HSD/hEDS. Pain-related fear is defined as fear that emerges when stimuli that are related to pain are perceived as a main threat, such as fear of movement, fear of injury or fear of pain ${ }^{15}$ and is assumed to play a core factor in the underlying mechanism explaining the level of disability in adolescents actually diagnosed with G-HSD/hEDS. Results of previous performed crosssectional studies showed that at least $75 \%$ of the individuals with G-HSD/hEDS had increased levels of fear of movement. ${ }^{16,17}$ Furthermore, fear of pain or injury were barriers to exercise and led to reduced or altered activities in individuals with G-HSD/hEDS. ${ }^{18,19}$ Also generalized anxiety and other fear-related psychiatric conditions, such as panic and anxiety disorders, have a high incidence in individuals with GHSD/hEDS (range 41.4\%-73.1\%). ${ }^{20-23}$ Thus, this higher incidence of pain-related fear and other anxiety-related problems are common in individuals with G-HSD/hEDS and might initiate the cascade of the cognitive/affective responses in the FAM leading to avoidance behaviour. As a consequence of hypermobility, adolescents are more vulnerable for developing CMP. And once being in pain, pain-related fear makes it even worse leading to a higher level of pain-related disability.

Avoidance behaviour aims at postponing or preventing a potential aversive situation. Adolescents with G-HSD/hEDS tend to avoid sports and outdoor games, probably due to an increased risk of injury and joint instability. Furthermore, adolescents with 
G-HSD/hEDS also report a higher need to rest. ${ }^{24}$ Even in adolescents with asymptomatic GJH results showed that these pain-free adolescents prefer more static activities, such as cycling and walking, and tend to avoid dynamic activities, such as sports. ${ }^{25}$ Although avoidance would suggest a lower level of activity, this assumption was not confirmed in a study in which the level of physical activity was measured based on accelerometry in adolescents with asymptomatic GJH compared to non-hypermobile controls. ${ }^{26}$ Furthermore, many individuals with GJH take advantage from their flexible joints and excel in sports and the performance arts. ${ }^{8,9}$ However, there is a difference in activityrelated behaviour in asymptomatic GJH compared to the behaviour in adolescents with G-HSD/hEDS. In adolescents with G-HSD/hEDS avoidance behaviour is maladaptive and seems to be a coping strategy to avoid (re)injury and prevent injury, and thus musculoskeletal pain. As proposed in the FAM, avoidance behaviour may lead to disability, depression, and disuse and eventually to physical deconditioning. Specifically, in adolescents with G-HSD/hEDS physical deconditioning has additional adverse consequences. Their compensation mechanism, essential for joint stability, might fail leading to proprioceptive impairment ${ }^{27-30}$ and decreased balance ${ }^{24,31}$, which in turn may lead to an increased risk of injury and affect gait patterns. ${ }^{31,32}$

Based on the applied FAM, it is proposed that higher levels of pain-related fear lead to a higher level of disability in adolescents with G-HSD/hEDS. Therefore, in the crosssectional study presented in chapter 3, it was evaluated whether the association between pain-related fear and disability in hypermobile adolescents with CMP differs from that in non-hypermobile adolescents with CMP. Findings of this study indicated that hypermobile adolescents with CMP had comparable levels of pain-related fear and perceived harmfulness of balance-related activities compared to non-hypermobile adolescents with CMP. Pain-related fear was measured with the Fear of Pain Questionnaire-Child report (FOPQ-C) and the perceived harmfulness of balance-related activities with an expert-developed subscale (PHODA-balance). In addition, although hypothesized, our study did not confirm that GJH and pain-related fear reinforced each other. No interaction effect could be identified between GJH and pain-related fear when testing their contribution to the perceived level of disability. Furthermore, the analysis did show that a higher level of pain-related fear was associated with a higher level of disability in adolescents with CMP, despite being hypermobile or not. This is in accordance with previous findings of the growing evidence on the negative role of painrelated fear in adolescents with $\mathrm{CMP} .33,34$

Results of earlier studies were inconclusive regarding the assumed lower physical functioning level in adolescents having asymptomatic GJH, which is a pain-free condition. Some studies indicated that asymptomatic GJH was associated with a decreased level of daily activity and muscle strength. ${ }^{35-37}$ However, other studies could 
not confirm a reduction in muscle strength and an altered physical activity level in individuals with asymptomatic GJH compared to non-hypermobile controls. ${ }^{26,38,39}$ Therefore, in chapter 4, it was evaluated whether adolescents with asymptomatic GJH have a lower level of physical functioning compared to non-hypermobile healthy adolescents. Furthermore, the potential role of pain-related fear on physical functioning was evaluated. The results showed that adolescents with asymptomatic GJH had comparable levels of physical functioning (measured as muscle strength, muscle strength endurance, motor performance, and physical activity level [PAL]) compared to non-hypermobile healthy adolescents. The level of perceived harmfulness, as measured with the Photograph Series of Daily Activities for youth (PHODA-youth), did not differ between adolescents with asymptomatic GJH and non-hypermobile controls. We need to consider that in the study presented in chapter 4, pain-related fear was measured using the PHODA-youth. In a pain-free population it is recommended to use a more generic tool, such as the Tampa Scale for Kinesiophobia (TSK), to measure pain-related fear. ${ }^{40}$ However, it should be noted that the psychometric properties of the TSK are questionable in identifying who is fearful. ${ }^{41}$ Therefore, the PHODA-youth might be more appropriate, since this instrument has very clear instructions how to specify activities/situations perceived as harmful for an adolescent and so the PHODA-youth provides information about which age-specific activities and social situations an adolescent fears or avoids. Further research is needed to evaluate the use of the PHODA-youth in a pain-free population.

In chapter 5 we evaluated whether adolescents with both CMP and GJH, adolescents with CMP and without GJH, adolescents without CMP but with $\mathrm{GJH}$, and adolescents without CMP or GJH differ in aspects of physical functioning, such as muscle strength, muscle strength endurance, motor performance, and PAL. Furthermore, we examined differences in pain-related fear between these groups and evaluated whether the potential contribution of pain-related fear on physical functioning varied between these groups. Results showed that adolescents with CMP, despite being hypermobile or not, had decreased knee flexion muscle strength, decreased knee extension and flexion muscle strength endurance, and lower motor performance related to balance, coordination, and joint stability compared to adolescents without CMP. This finding is independent of the finding whether an adolescent is also having GJH or not. This reduction in functioning of adolescents with CMP was reported before. Previous studies showed decreased knee, hip, back, and abdominal muscle strength and a reduction in motor performance in adolescents with CMP compared to pain-free controls. ${ }^{3,42,43}$ These studies included populations with age and diagnosis in accordance with our population. In addition, also the reported scores on self-reported measures of disability (Functional Disability Inventory [FDI], average score of $23.8 \pm 10.2$ ) in the study of Sil et al. ${ }^{43}$ were 
comparable to results in our study (average $23.8 \pm 9.4$ ). Both can be classified as moderately disabled (range 13-29). ${ }^{44}$ A discrepancy between the studies did however exist, two of the three studies had a higher percentage of boys in their population (40-54\%) compared to the population in this study in chapter $5(10 \%){ }^{3,42}$ Nevertheless, it is important to consider that the decreased muscle strength and lower motor performance as identified might also be due to pain experienced during testing and/or pain-related fear. As a result, adolescents with CMP might decide to stop prematurely or perform submaximal, resulting in a lower score. ${ }^{45} \mathrm{~A}$ remarkable finding is that results of chapter 5 also indicated that (hypermobile and non-hypermobile) adolescents with CMP and pain-free controls had comparable physical activity levels, despite the discrepancy in muscle strength and motor performance between these groups. Data from 1.6 million school-going adolescents (aged 11-17 years) worldwide showed that the majority $(77.6 \%$ boys and $84.7 \%$ girls) did not meet current physical activity guidelines according to the WHO recommendation (at least 60 min of daily physical activity of moderate-to-vigorous intensity). ${ }^{46}$ This might imply that adolescents nowadays are less active and that possible differences in daily life activities related to CMP are not that distinctive. The finding that the level of daily life activities assessed with accelerometry did not differ between adolescents with CMP and/or GJH compared to pain-free controls and/or GJH was in accordance with results reported in adults with pain. ${ }^{47}$ This could suggest that, if restoring a normal pattern of daily functioning would be the main outcome of multidisciplinary rehabilitation treatment; adolescents with CMP and/or GJH are not eligible for multidisciplinary rehabilitation treatment. However, the main aim of multidisciplinary rehabilitation treatment is far more than restoring a normal pattern of daily functioning and should focus on reducing disability in terms of activity limitations and participation restrictions, improving quality of life and societal functioning, as well as participation in sports and leisure activities. Furthermore, multidisciplinary rehabilitation treatment should be adapted based on a patient's needs and context. Therefore, adolescents with G-HSD/hEDS experiencing disability, a lower quality of life and/or restricted societal functioning are indeed eligible for multidisciplinary rehabilitation treatment. The finding of unchanged physical activity levels will even more emphasize that the decrease in physical functioning (such as muscle strength and endurance, and motor performance) in adolescents with CMP, despite being hypermobile or not, could be due to pain-related fear negatively influencing test scores of physical functioning.

Earlier findings in the literature did however show that psychological distress, such as anxiety and depression, indeed had a significant impact on disability in individuals with G-HSD/hEDS, indicating higher levels of disability with increasing levels of psychological distress. ${ }^{48}$ Furthermore, the results of chapter 3 already showed that pain-related fear was not more pronounced in hypermobile adolescents with CMP compared to non- 
hypermobile adolescents with CMP. Taken together these findings with results of chapter 3 and 5 seem to imply that in the chronicity of musculoskeletal pain the level of pain-related fear is associated with disability or decreased physical functioning, despite being hypermobile or not. These results do not confirm our hypothesis that pain-related fear would be more prominent in adolescents with G-HSD/hEDS compared to nonhypermobile adolescents with CMP as presented in the applied FAM. A possible explanation could be that the selected adolescents with CMP in the studies presented in chapter 3 and 5, who were referred to the university outpatient rehabilitation clinic specialised in pain rehabilitation, already experience long-term disabling pain complaints resulting in a decline of daily functioning and physical deconditioning in both the hypermobile and non-hypermobile group. As has been shown in earlier studies, being hypermobile will make an individual more vulnerable to develop CMP. ${ }^{6,7}$ The increased prevalence of GJH in the CMP population (28-35\%) compared to the pain-free population (18\%) seems in line with the fact that hypermobility can be considered as a risk factor for getting CMP. However, once an individual experiences CMP, especially psychosocial factors, such as pain-related fear and catastrophizing, seem to contribute to a decrease in physical functioning or an increase in disability, and not hypermobility. In chapter 6, in a pre-post-test design study, changes in the level of disability, physical functioning, perceived harmfulness, and pain intensity as a result of completing multidisciplinary rehabilitation treatment in adolescents with G-HSD/hEDS were found. According to the applied FAM (chapter 2), in which it is hypothesized that GJH and painrelated fear are important factors and can even strengthen each other in the development and maintenance of disabling CMP, the treatment consisted of physical training and a targeted cognitive-behavioural therapy intervention; exposure in vivo (EXP). Physical training focused on improving aerobic capacity, muscle strength, and propriocepsis to compensate for the physical negative consequences in adolescents with G-HSD/hEDS. EXP focused on reducing pain-related fear, by exposing the adolescents to activities of daily living and movements to test catastrophic cognitions. Results showed that this multidisciplinary rehabilitation treatment leads to a significantly and clinically relevant improvement (67\%) in the level of functional disability $(r=-0.61)$. Furthermore, muscle strength, motor performance, perceived harmfulness, and pain intensity improved significantly ( $r$ between -0.45 to -0.62 ). Despite the fact that the main goal of this multidisciplinary rehabilitation treatment was to reduce the level of disability, the $63 \%$ reduction in pain intensity is remarkable. In adolescents with CMP, a multidisciplinary rehabilitation treatment consisted of only EXP demonstrated positive effects on functional disability, pain-related fear, pain catastrophizing, and pain intensity. ${ }^{49}$ However, the reduction in pain intensity (33\%) after EXP was far more limited as compared to the $63 \%$ pain decrease as a result of the combined treatment of physical 
training and EXP. This increased reduction in pain intensity for the combined treatment could be an additional effect of the physical training in this hypermobile population. A potential explanation could be that physical training may result in improvements in propriocepsis, aerobic capacity, and muscle strength in adolescents with G-HSD/hEDS and might restore the compensation mechanism, essential for joint stability to compensate for joint laxity, as proposed in the applied FAM. These physical improvements can potentially lead to better motor control and balance and might play a protective role in micro (re)injuries, potentially leading to a lower level of pain. The results of chapter 6 , although based on a small sample size and a pre-post-test design, seem to indicate that multidisciplinary rehabilitation treatment is indeed a legitimate treatment for reducing disability by focussing on the different elements of the applied FAM (chapter 2). This leads to the remarkable finding, that although the studies in chapter 3 and 5 did not confirm our hypothesis underlying the applied FAM that painrelated fear would be more prominent in adolescents with G-HSD/hEDS, our intervention seem to improve both physical and psychosocial factors. How can these contradicting outcomes of the different chapters further unravel the complex puzzle of disabling CMP in adolescents with G-HSD/hEDS and lead to clinical implications in daily practice?

\section{Implications for clinical practice}

The result of this dissertation contribute to identify which adolescents might be at risk for persistent pain and pain-related disability. A previous study showed that adolescents with functional abdominal pain and higher levels of pain-related anxiety are at risk of persistent pain and disability throughout adulthood. ${ }^{50}$ The data presented in this dissertation showed that pain-related fear is associated with disability (chapter 3) and lower physical functioning levels (chapter 5) in adolescents with CMP, despite being hypermobile or not, compared to adolescents without CMP. In addition, results from pain-free adolescents with GJH (chapter 4) showed no altered physical functioning levels and no different level of pain-related fear as compared to non-hypermobile pain-free adolescents. Since especially pain-related fear seems to be disabling, early detection of pain-related fear in primary care, for instance by a GP or paediatrician or paediatric physical therapist, is warranted. Combining this with prognostic factors and risk factors for chronic pain may identify adolescents with CMP in an early stage who are at risk to further develop disabling CMP. One of the measurements that can be used to detect pain-related fear in adolescents with CMP is the Fear of Pain Questionnaire-Child version (FOPQ-C), which is also recently validated in the Dutch language. ${ }^{51}$ Early intervention of pain-related fear is likely to improve pain-related outcomes and may be potentially protective for long-term outcomes. 
Many Dutch patients with CMP perceived their treatment as inadequate. ${ }^{52} \mathrm{~A}$ reason could be that the complexity of the patient's pain problem does not match the treatment delivered by the health care professionals, resulting in an ineffective treatment and unnecessary costs. Therefore, it is recommended that health care professionals in primary care thoroughly assess the adolescent's pain problem, identify the adolescent's needs, be aware of the influencing psychosocial factors, such as painrelated fear, and if applicable make use of generic and specific measurements. After this assessment, a decision can be made matching the complexity of the pain problem and allowing the adolescent to get the right care at the right time. And this means, in case of minimal disability in daily life activities with only restricted influence of psychosocial factors, health care professionals in primary care should use a biopsychosocial approach and focus on supporting the adolescent to get active and functioning age appropriate despite pain. ${ }^{1}$ However, once adolescents with CMP, despite being hypermobile or not, have moderate to severe levels of disability in daily life activities and associated influencing psychosocial factors multidisciplinary rehabilitation treatment is recommended.

The pre-post-test study (chapter 6) showed that multidisciplinary rehabilitation treatment consisted out of physical training and EXP led to significant improvements in disability, physical functioning, perceived harmfulness, and pain intensity in adolescents with G-HSD/hEDS. Previous studies, solely focusing on exercise as a cornerstone of the treatment in adults with G-HSD/hEDS, also showed post-treatment improvements in pain intensity and indicated this as a result of improvement in propriocepsis. ${ }^{27,53}$ These studies were conducted in a predominantly adult population to which a 8-week exercise programme mainly consisting out of proprioceptive exercises was provided. In contrast, our intervention was designed for an adolescent population consisted of a 8-week physical training module also aimed to improve aerobic capacity and muscle strength, alongside propriocepsis. The cardiovascular and musculoskeletal training parameters in our study were based on criteria of the American College of Sports Medicine and the National Strength and Conditioning Association for adolescents. ${ }^{54,55}$ Other uncontrolled clinical studies used multidisciplinary treatments in adults with G-HSD/hEDS combining physical therapy and cognitive-behavioural therapy, but did not specifically focus on reducing pain-related fear. ${ }^{56,57}$ Improvements were found in several outcomes (such as perceived performance of daily activities, muscle strength, self-efficacy, and depression) covering both physical and psychological factors, suggesting that multidisciplinary treatment with a cognitive-behavioural approach might be feasible for adults with GHSD/hEDS. Unfortunately, these studies overall are limited in size and quality and need further evidence with more rigorous research designs. ${ }^{58,59}$ 
The results in this dissertation seemed to imply that in future multidisciplinary rehabilitation treatment for adolescents with G-HSD/hEDS, who have moderate to severe disability, it is important to focus also on psychological components, such as painrelated fear, catastrophizing thoughts, and generalized anxiety. We would like to propose a model in which the different elements during the analysis phase and the potential multidisciplinary rehabilitation treatments elements are presented (Figure 7.2).

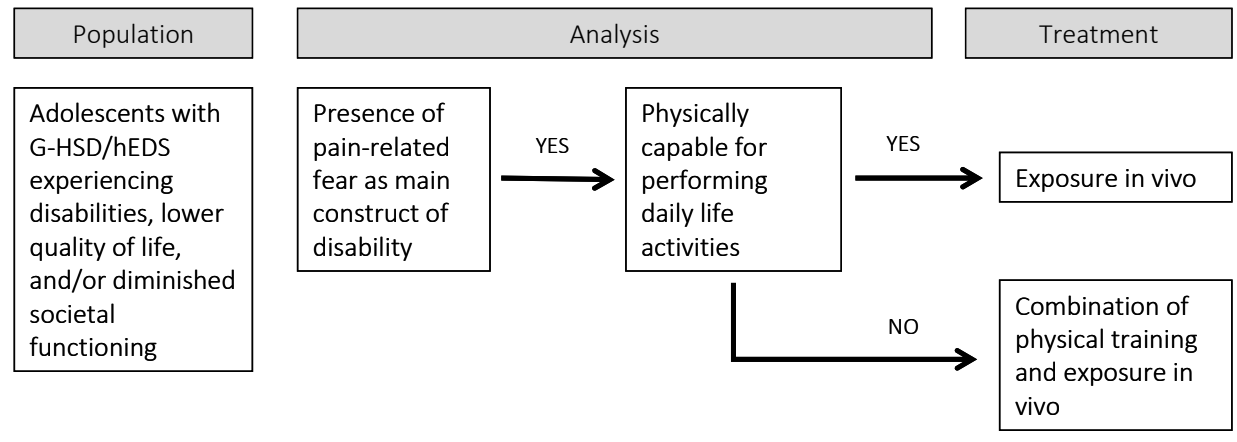

Figure 7.2 Multidisciplinary rehabilitation treatment for adolescents with G-HSD/hEDS and considerable disability.

During the analysis phase the different constructs related to disability should be explored. The presence of pain-related fear as the main construct of disability needs to be identified. Questionnaires such as the FOPQ-C and the PHODA-youth can be helpful for the multidisciplinary rehabilitation team. Furthermore, as mentioned earlier, physical deconditioning as a result of long-term avoidance behaviour and lower activity levels might lead to additional physical negative consequences, such as decreased muscle strength, cardiorespiratory fitness, and balance in adolescents with G-HSD/hEDS. These lower scores on objectively assessed physical functioning measures are hypothesized to deteriorate the compensation mechanism essential for joint stability. Therefore, these lower scores might increase the risks of (re)injury and falls causing the onset of a new episode of musculoskeletal pain and perpetuating the vicious circle of disabling pain and thus essential to address during the analysis phase. In addition, the physiotherapist should be aware of pain experience and pain-related fear during the assessment. Therefore, adolescents with G-HSD/hEDS might decide to stop or perform submaximally, resulting in a lower physical score, which is not representative for the physical capabilities.

Afterwards, a tailored multidisciplinary rehabilitation treatment can be chosen based on the analysis performed. If pain-related fear is present and the adolescents with G-HSD/hEDS are physically capable of performing daily life activities, EXP might be the 
preferable intervention to restore normal pattern of daily functioning by reducing painrelated fear and catastrophic thinking. However, if decreased physical functioning components (such as muscle strength, cardiorespiratory fitness, and balance) are present which interfere with the capability of performing daily life activities, multidisciplinary rehabilitation treatment should contain a specific condition-creating physical training focusing on improving the adverse physical components due to deconditioning followed by EXP.

From a clinical perspective, many adolescents with G-HSD/hEDS who received the combined treatment of physical training and EXP reported already an increase in selfesteem during the physical training weeks and translated this in increased performance of valuable daily life activities. These adolescents might have already experienced a decrease in pain-related fear and catastrophic thoughts before the start of EXP. In addition, the increase of self-esteem might also be due to a reduction of pain intensity that could potentially be started to decrease as a result of the physical training. Pain reduction could also be due to a decreased attention towards pain. A recent study showed that low self-esteem could be an important factor in the maintenance of chronic

pain in adolescents. ${ }^{60}$ Furthermore, during adolescence lower levels of self-esteem were associated with higher levels of anxiety and depressive symptoms ${ }^{61}$, which are both associated with CMP. ${ }^{62}$ Therefore, measuring self-esteem could be of interest in further studies and clinical practice. Unfortunately, we only measured before and after the multidisciplinary rehabilitation treatment and did not assess the situation in between: after completion of the physical training before EXP starts. Therefore we cannot determine the exact level of pain reduction during the physical training part alone. In future, it would be favourable to add measurements at the end of the physical training. Furthermore, follow-up measurements are warranted to determine changes over time.

\section{Methodological considerations}

The studies presented in this dissertation focused on the role of having GJH, having CMP, and the potential influence of pain-related fear on physical functioning and disability within adolescents with G-HSD/hEDS. Therefore, these studies contribute to further unravel the complex puzzle of disabling CMP in adolescents with G-HSD/hEDS. Better understanding leads to better tailored and personalised interventions. In the following paragraphs we will focus on a number of methodological issues that we encountered.

A limitation to this work is the result of a major change in the diagnostic criteria of G-HSD/hEDS in 2017, the former joint hypermobility syndrome (JHS)/Ehlers-Danlos syndrome hypermobility type (EDS-HT). These new diagnostic criteria led to a change in the classification and nomenclature of symptomatic GJH-related disorders according to updated criteria and cut-off points of the BS. The updated criteria recommended now 
for clinical use in adults a BS with a cut-off point of 5 out of 9 and in children and adolescents up to 18 years a BS with a cut-off point of 6 out of $9 .^{63}$ However, the crosssectional study described in chapter 3 was already conducted according to the former criteria with a cut-off point of 5 out of 9 of the BS for all adolescents (aged 11-21 years). ${ }^{64}$ In case updated criteria would be applied, the prevalence of hypermobility in adolescents with CMP changed from 35\% (40/116) to 26\% (30/116) in chapter 3. Additionally, new criteria would not result in a significant change in findings for the study as presented in chapter 3 . This percentage of hypermobility $(26 \%)$ identified based on new calculations on data in the study of chapter 3 might be comparable to the findings in chapter 5, in which 9 of the 30 adolescents with CMP had GJH (28\%). Furthermore, as mentioned before we used G-HSD/hEDS in this dissertation as an umbrella term to cover the new and old nomenclature of GJH-related disorders with CMP. Therefore, it should be noted that it is currently unknown what the consequences are of these new unified criteria for patient outcomes and disease characteristics.

Although the BS is the most widely used tool for identifying GJH in clinical practice and is considered to be a reliable measure ${ }^{63}$, the validity of the BS for identifying whole body hypermobility is still a topic for debate. The BS is dominated by tests of the upper extremity. As two out of nine scores, only (double sided) knee extension is included as a test for hypermobility related to the lower limb. ${ }^{65}$ In addition, the BS does not include tests for the shoulder or radioulnar joint, whereas the shoulder is most commonly identified as unstable in patients with G-HSD/hEDS ${ }^{66}$ and the radioulnar joint is a common affected body region. ${ }^{67}$ Recently, other instruments such as the Lower Limb Assessment Scale and the Upper Limb Hypermobility Assessment Tool specific addressing multiple joints are reliable and valid measurements in identifying lower limb and upper limb hypermobility, and GJH. ${ }^{68,69}$ The clinical use of these tools seems evident when individuals present predominantly lower or upper limb symptoms. Further research is needed to determine the ideal test or combination of tests to identify GJH.

Another limitation to this work is the cross-sectional design we used in studies presented in chapter 3, 4 and 5. And in addition, also the studies reported in chapter 2 that support the individual associations within the hypothesized applied FAM were mostly crosssectional in nature, with only a few evaluating more than one aspect of the FAM. Therefore, no causal relationship could be confirmed. However, at this moment there are only a limited number of studies with a longitudinal design conducted in adolescents with G-HSD/hEDS and evidence on the potential role of pain-related fear is scarce. Therefore, these studies adds to further unravel the role of pain-related fear in the mechanism of disabling G-HSD/hEDS. In addition, the pre-post-test design, used in chapter 6, lacks a control group. Again, this limits the ability to infer causality of any improvements observed as a single effect of participation in multidisciplinary 
rehabilitation treatment. However, this feasibility study could be the prelude to further studies with more rigorous research designs. Furthermore, the findings of the studies, described in chapter 4, 5 and 6, are based on rather small sample sizes within the asymptomatic GJH and G-HSD/hEDS population, that could have resulted into bias. However, to our opinion, this assumption seems not to be the case, since the percentage of hypermobility in the patient groups is comparable to that in other studies, despite the different cut-off points of the BS.

We need to consider that studies in this dissertation included adolescents with longterm disabling pain complaints, in an university outpatient rehabilitation clinic specialised in pain rehabilitation. Therefore, our results might be specific to this population in this setting and this is important to consider when generalizing the results to other populations or settings.

The focus of this dissertation is specifically on pain-related fear and its relation to disability in adolescents with G-HSD/hEDS. This focus is chosen, since we used the theoretical FAM as a framework for our hypotheses. As a result, other important constructs in explaining disability, such as multi-systemic dysfunction and fatigue, were not included. ${ }^{70}$ There is also growing evidence of autonomic nervous system dysfunction in G-HSD/hEDS contributing to disability and decreased quality of life. ${ }^{71,72}$ Furthermore, parental/family influences have a strong influence on adolescent pain experience, maintenance of pain behaviour, and disability. ${ }^{73,74}$ Therefore, health care professionals should be aware of the various constructs in explaining disability in adolescent with G-HSD/hEDS. Although recognized as important, these factors were beyond the scope of this dissertation.

\section{Recommendations for future research}

To further unravel the complexity of disabling CMP in adolescents with G-HSD/hEDS several suggestions for further research can be given.

The cross-sectional studies in this dissertation showed that pain-related fear is an important determinant of a decrease in physical functioning and disability in adolescents with G-HSD/hEDS. Furthermore, in adolescents with asymptomatic GJH compared to non-hypermobile controls no differences in physical functioning and pain-related fear were found. More insight in physical and psychological functioning of adolescents with $\mathrm{GJH}$, especially before the onset of CMP, will help to unravel the influence of GJH, physical and psychological functioning in adolescents who will or will not develop CMP. Therefore, longitudinal studies are warranted to examine associations over time.

Furthermore, the results of the pre-post-test design showed improvements in disability, pain-related fear, physical functioning, and pain intensity and are promising. Therefore, new studies are warranted to further investigate the effectiveness of this 
multidisciplinary rehabilitation treatment. An RCT is considered as the gold standard to evaluate treatment effectiveness. However, RCT's are time consuming, the execution requires funding and resources, and struggles with sample size are common difficulties. A favourable design could be single-case design which allows for tailoring and customizing the multidisciplinary rehabilitation treatment. Each patient functions as its own control and single-case designs are considered to be more compatible with clinical practice than a RCT. ${ }^{75}$ Furthermore, a lower sample size is required and this is preferable in a less prevalent condition such as G-HSD/hEDS. Thus, a single case design would be recommended to further investigate the effectiveness of the combined treatment of physical training and EXP. In addition, further studying the tailored multidisciplinary rehabilitation treatment based on the analysis performed as proposed in Figure 7.2 could be of interest.

Finally, more research is needed that incorporates other constructs related to disability in adolescents with G-HSD/hEDS. As described previously fatigue, multi-systemic dysfunction, autonomic dysfunction, and parental influence are constructs who might contribute to explaining disability. Better understanding of these factors and recognition in clinical practice allows to better identify adolescents who may be vulnerable for disabling pain and might lead to tailored interventions aimed at decreasing pain-related disabilities and increasing quality of life.

\section{General conclusion}

This dissertation showed that pain-related fear is an important determinant of a decrease in physical functioning and disability in adolescents with CMP, despite being hypermobile or not. Furthermore, adolescents with asymptomatic GJH had no lower level of physical functioning and experienced no pain-related fear compared to nonhypermobile controls. The intervention study, consisting out of a multidisciplinary rehabilitation treatment with physical training and EXP showed improvements in disability, physical functioning, perceived harmfulness, and pain-intensity in adolescents with G-HSD/hEDS. More tailored multidisciplinary rehabilitation treatments are needed to better enable these adolescents to optimally participate in society. 


\section{References}

1. Gatchel RJ, Peng YB, Peters ML, Fuchs PN, Turk DC. The biopsychosocial approach to chronic pain: scientific advances and future directions. Psychol Bull. 2007;133(4):581-624.

2. Kashikar-Zuck S. Treatment of children with unexplained chronic pain. Lancet. 2006;367(9508):380-2.

3. O'Sullivan P, Beales D, Jensen L, Murray K, Myers T. Characteristics of chronic non-specific musculoskeletal pain in children and adolescents attending a rheumatology outpatients clinic: a crosssectional study. Pediatr Rheumatol Online J. 2011;9(1):3.

4. Martin AL, McGrath PA, Brown SC, Katz J. Anxiety sensitivity, fear of pain and pain-related disability in children and adolescents with chronic pain. Pain Research and Management. 2007;12(4):267-72.

5. Simons LE, Sieberg CB, Carpino E, Logan D, Berde C. The Fear of Pain Questionnaire (FOPQ): assessment of pain-related fear among children and adolescents with chronic pain. J Pain. 2011;12(6):677-86.

6. Sohrbeck-Nohr O, Kristensen JH, Boyle E, Remvig L, Juul-Kristensen B. Generalized joint hypermobility in childhood is a possible risk for the development of joint pain in adolescence: a cohort study. BMC Pediatr. 2014;14:302.

7. Tobias JH, Deere K, Palmer S, Clark EM, Clinch J. Joint hypermobility is a risk factor for musculoskeletal pain during adolescence: findings of a prospective cohort study. Arthritis Rheum. 2013;65(4):1107-15.

8. Scheper MC, de Vries JE, de Vos R, Verbunt J, Nollet F, Engelbert RH. Generalized joint hypermobility in professional dancers: a sign of talent or vulnerability? Rheumatology (Oxford). 2013;52(4):651-8.

9. Larsson LG, Baum J, Mudholkar GS, Kollia GD. Benefits and disadvantages of joint hypermobility among musicians. N Engl J Med. 1993;329(15):1079-82.

10. Tinkle B, Castori M, Berglund B, Cohen H, Grahame R, Kazkaz H, et al. Hypermobile Ehlers-Danlos syndrome (a.k.a. Ehlers-Danlos syndrome Type III and Ehlers-Danlos syndrome hypermobility type): Clinical description and natural history. Am J Med Genet C Semin Med Genet. 2017;175(1):48-69.

11. Castori M, Tinkle B, Levy H, Grahame R, Malfait F, Hakim A. A framework for the classification of joint hypermobility and related conditions. Am J Med Genet C Semin Med Genet. 2017;175(1):148-57.

12. Malfait F, Francomano C, Byers P, Belmont J, Berglund B, Black J, et al. The 2017 international classification of the Ehlers-Danlos syndromes. Am J Med Genet C Semin Med Genet. 2017;175(1):8-26.

13. Simons LE, Kaczynski KJ. The Fear Avoidance model of chronic pain: examination for pediatric application. J Pain. 2012;13(9):827-35.

14. Vlaeyen JW, Linton SJ. Fear-avoidance and its consequences in chronic musculoskeletal pain: a state of the art. Pain. 2000;85(3):317-32.

15. Leeuw M, Goossens ME, Linton SJ, Crombez G, Boersma K, Vlaeyen JW. The fear-avoidance model of musculoskeletal pain: current state of scientific evidence. J Behav Med. 2007;30(1):77-94.

16. Baeza-Velasco C, Bourdon C, Montalescot L, de Cazotte C, Pailhez G, Bulbena A, et al. Low- and highanxious hypermobile Ehlers-Danlos syndrome patients: comparison of psychosocial and health variables. Rheumatol Int. 2018.

17. Celletti C, Castori M, La Torre G, Camerota F. Evaluation of kinesiophobia and its correlations with pain and fatigue in joint hypermobility syndrome/Ehlers-Danlos syndrome hypermobility type. Biomed Res Int. 2013;2013:580460.

18. Schmidt A, Corcoran K, Grahame R, de CWAC. How do people with chronically painful joint hypermobility syndrome make decisions about activity? Br J Pain. 2015;9(3):157-66.

19. Simmonds JV, Herbland A, Hakim A, Ninis N, Lever W, Aziz Q, et al. Exercise beliefs and behaviours of individuals with Joint Hypermobility syndrome/Ehlers-Danlos syndrome - hypermobility type. Disabil Rehabil. 2017:1-11.

20. Bulbena A, Gago J, Pailhez G, Sperry L, Fullana MA, Vilarroya O. Joint hypermobility syndrome is a risk factor trait for anxiety disorders: a 15-year follow-up cohort study. Gen Hosp Psychiatry. 2011;33(4): 363-70.

21. Bulbena-Cabre A, Duno L, Almeda S, Batlle S, Camprodon-Rosanas E, Martin-Lopez LM, et al. Joint hypermobility is a marker for anxiety in children. Rev Psiquiatr Salud Ment. 2019;12(2):68-76.

22. Garcia Campayo J, Asso E, Alda M, Andres EM, Sobradiel N. Association between joint hypermobility syndrome and panic disorder: a case-control study. Psychosomatics. 2010;51(1):55-61. 
23. Javadi Parvaneh V, Modaress S, Zahed G, Rahmani K, Shiari R. Prevalence of generalized joint hypermobility in children with anxiety disorders. BMC Musculoskelet Disord. 2020;21(1):337.

24. Schubert-Hjalmarsson E, Ohman A, Kyllerman M, Beckung E. Pain, balance, activity, and participation in children with hypermobility syndrome. Pediatr Phys Ther. 2012;24(4):339-44.

25. Scheper M, de Vries J, Beelen A, de Vos R, Nollet F, Engelbert R. Generalized joint hypermobility, muscle strength and physical function in healthy adolescents and young adults. Curr Rheumatol Rev. 2014;10(2):117-25.

26. Clinch J, Deere K, Sayers A, Palmer S, Riddoch C, Tobias JH, et al. Epidemiology of generalized joint laxity (hypermobility) in fourteen-year-old children from the UK: a population-based evaluation. Arthritis Rheum. 2011;63(9):2819-27.

27. Sahin N, Baskent A, Cakmak A, Salli A, Ugurlu H, Berker E. Evaluation of knee proprioception and effects of proprioception exercise in patients with benign joint hypermobility syndrome. Rheumatol Int. 2008;28(10):995-1000.

28. Fatoye F, Palmer S, Macmillan F, Rowe P, van der Linden M. Proprioception and muscle torque deficits in children with hypermobility syndrome. Rheumatology (Oxford). 2009;48(2):152-7.

29. Rombaut L, De Paepe A, Malfait F, Cools A, Calders P. Joint position sense and vibratory perception sense in patients with Ehlers-Danlos syndrome type III (hypermobility type). Clin Rheumatol. 2010;29(3): 289-95.

30. Smith TO, Jerman E, Easton V, Bacon H, Armon K, Poland F, et al. Do people with benign joint hypermobility syndrome (BJHS) have reduced joint proprioception? A systematic review and metaanalysis. Rheumatol Int. 2013;33(11):2709-16.

31. Rombaut L, Malfait F, De Wandele I, Thijs Y, Palmans T, De Paepe A, et al. Balance, gait, falls, and fear of falling in women with the hypermobility type of Ehlers-Danlos syndrome. Arthritis Care Res (Hoboken). 2011;63(10):1432-9.

32. Scheper MC, de Vries JE, Verbunt J, Engelbert RH. Chronic pain in hypermobility syndrome and EhlersDanlos syndrome (hypermobility type): it is a challenge. J Pain Res. 2015;8:591-601.

33. Caes L, Fisher E, Clinch J, Tobias JH, Eccleston C. The role of pain-related anxiety in adolescents' disability and social impairment: ALSPAC data. Eur J Pain. 2015;19(6):842-51.

34. Fisher E, Heathcote LC, Eccleston C, Simons LE, Palermo TM. Assessment of Pain Anxiety, Pain Catastrophizing, and Fear of Pain in Children and Adolescents With Chronic Pain: A Systematic Review and Meta-Analysis. J Pediatr Psychol. 2018;43(3):314-25.

35. Jindal P, Narayan A, Ganesan S, MacDermid JC. Muscle strength differences in healthy young adults with and without generalized joint hypermobility: a cross-sectional study. BMC Sports Sci Med Rehabil. 2016;8:12.

36. Scheper M, de Vries J, Beelen A, de Vos R, Nollet F, Engelbert R. Generalized joint hypermobility, muscle strength and physical function in healthy adolescents and young adults. Curr Rheumatol Rev. 2014;10(2):117-25.

37. Scheper MC, de Vries JE, Juul-Kristensen B, Nollet F, Engelbert RH. The functional consequences of generalized joint hypermobility: a cross-sectional study. BMC Musculoskeletal Disorders. 2014;15:243.

38. Jensen BR, Olesen AT, Pedersen MT, Kristensen JH, Remvig L, Simonsen EB, et al. Effect of generalized joint hypermobility on knee function and muscle activation in children and adults. Muscle Nerve. 2013;48(5):762-9.

39. Mebes C, Amstutz A, Luder G, Ziswiler HR, Stettler M, Villiger PM, et al. Isometric rate of force development, maximum voluntary contraction, and balance in women with and without joint hypermobility. Arthritis \& Rheumatism. 2008;59(11):1665-9.

40. Houben RM, Leeuw M, Vlaeyen JW, Goubert L, Picavet HS. Fear of movement/injury in the general population: factor structure and psychometric properties of an adapted version of the Tampa Scale for Kinesiophobia. J Behav Med. 2005;28(5):415-24.

41. Lundberg M, Grimby-Ekman A, Verbunt J, Simmonds MJ. Pain-related fear: a critical review of the related measures. Pain Res Treat. 2011;2011:494196.

42. Jones MA, Stratton $G$, Reilly T, Unnithan VB. Biological risk indicators for recurrent non-specific low back pain in adolescents. Br J Sports Med. 2005;39(3):137-40.

43. Sil S, Thomas S, DiCesare C, Strotman D, Ting TV, Myer G, et al. Preliminary evidence of altered biomechanics in adolescents with juvenile fibromyalgia. Arthritis Care Res (Hoboken). 2015;67(1):102-11. 
44. Kashikar-Zuck S, Flowers SR, Claar RL, Guite JW, Logan DE, Lynch-Jordan AM, et al. Clinical utility and validity of the Functional Disability Inventory among a multicenter sample of youth with chronic pain. Pain. 2011;152(7):1600-7.

45. Huijnen IPJ, Verbunt J, Wittink HM, Smeets RJEM. Physical performance measurement in chronic low back pain: measuring physical capacity of pain-related behaviour? European Journal of Physiotherapy. 2013;15(3):103-10.

46. Guthold R, Stevens GA, Riley LM, Bull FC. Global trends in insufficient physical activity among adolescents: a pooled analysis of 298 population-based surveys with 1.6 million participants. Lancet Child Adolesc Health. 2020;4(1):23-35.

47. Verbunt JA, Westerterp KR, van der Heijden GJ, Seelen HA, Vlaeyen JW, Knottnerus JA. Physical activity in daily life in patients with chronic low back pain. Arch Phys Med Rehabil. 2001;82(6):726-30.

48. Scheper MC, Juul-Kristensen B, Rombaut L, Rameckers EA, Verbunt J, Engelbert RH. Disability in Adolescents and Adults Diagnosed With Hypermobility-Related Disorders: A Meta-Analysis. Arch Phys Med Rehabil. 2016;97(12):2174-87.

49. van den Bogert - Dekker C. Adolescent Chronic Pain. Rehabilitation treatment targeting pain-related fear [dissertation]. Heerlen (NL): Maastricht University; 2018.

50. Walker LS, Sherman AL, Bruehl S, Garber J, Smith CA. Functional abdominal pain patient subtypes in childhood predict functional gastrointestinal disorders with chronic pain and psychiatric comorbidities in adolescence and adulthood. Pain. 2012;153(9):1798-806.

51. Dekker C, Bastiaenen CHG, de Vries JE, Simons LE, Goossens M, Verbunt J. Dutch version of the Fear of Pain Questionnaire for adolescents with chronic pain. Disabil Rehabil. 2018;40(11):1326-32.

52. Bekkering GE, Bala MM, Reid K, Kellen E, Harker J, Riemsma R, et al. Epidemiology of chronic pain and its treatment in The Netherlands. Neth J Med. 2011;69(3):141-53.

53. Ferrell WR, Tennant N, Sturrock RD, Ashton L, Creed G, Brydson G, et al. Amelioration of symptoms by enhancement of proprioception in patients with joint hypermobility syndrome. Arthritis Rheum. 2004; 50(10):3323-8.

54. Faigenbaum AD, Kraemer WJ, Blimkie CJ, Jeffreys I, Micheli LJ, Nitka M, et al. Youth resistance training: updated position statement paper from the national strength and conditioning association. J Strength Cond Res. 2009;23(5 Suppl):S60-79.

55. Faigenbaum AD, Myer GD. Pediatric resistance training: benefits, concerns, and program design considerations. Curr Sports Med Rep. 2010;9(3):161-8.

56. Bathen $T$, Hangmann AB, Hoff M, Andersen LO, Rand-Hendriksen S. Multidisciplinary treatment of disability in ehlers-danlos syndrome hypermobility type/hypermobility syndrome: A pilot study using a combination of physical and cognitive-behavioral therapy on 12 women. Am J Med Genet A. 2013; 161(12):3005-11.

57. Rahman A, Daniel C, Grahame R. Efficacy of an out-patient pain management programme for people with joint hypermobility syndrome. Clin Rheumatol. 2014;33(11):1665-9.

58. Palmer S, Davey I, Oliver L, Preece A, Sowerby L, House S. The effectiveness of conservative interventions for the management of syndromic hypermobility: a systematic literature review. Clin Rheumatol. 2020.

59. Engelbert RH, Juul-Kristensen B, Pacey V, de Wandele I, Smeenk S, Woinarosky N, et al. The evidencebased rationale for physical therapy treatment of children, adolescents, and adults diagnosed with joint hypermobility syndrome/hypermobile Ehlers Danlos syndrome. Am J Med Genet C Semin Med Genet. 2017;175(1):158-67.

60. Shaygan M, Karami Z. Chronic Pain in Adolescents: The Predictive Role of Emotional Intelligence, SelfEsteem and Parenting Style. Int J Community Based Nurs Midwifery. 2020;8(3):253-63.

61. Sowislo JF, Orth U. Does low self-esteem predict depression and anxiety? A meta-analysis of longitudinal studies. Psychol Bull. 2013;139(1):213-40.

62. Eccleston C, Crombez G, Scotford A, Clinch J, Connell H. Adolescent chronic pain: patterns and predictors of emotional distress in adolescents with chronic pain and their parents. Pain. 2004;108(3):221-9.

63. Juul-Kristensen B, Schmedling K, Rombaut L, Lund H, Engelbert RH. Measurement properties of clinical assessment methods for classifying generalized joint hypermobility-A systematic review. Am J Med Genet C Semin Med Genet. 2017;175(1):116-47.

64. Smits-Engelsman B, Klerks M, Kirby A. Beighton score: a valid measure for generalized hypermobility in children. J Pediatr. 2011;158(1):119-23. 
65. Ferrari J, Parslow C, Lim E, Hayward A. Joint hypermobility: the use of a new assessment tool to measure lower limb hypermobility. Clin Exp Rheumatol. 2005;23(3):413-20.

66. Hakim AJ, Malfait F, de Paepe A. The heritable disorders of connective tissue: epidemiology, nosology and clinical features. In: Hakim AJ, Keer R, Grahame R, editors. Hypermobility, Fibromyalgia and Chronic Pain: Churchill Livingstone/Elsevier; 2010. p. 3-17.

67. Quarrier NF. Is hypermobility syndrome (HMS) a contributing factor for chronic unspecific wrist pain in a musician? If so, how is it evaluated and managed? Work. 2011;40(3):325-33.

68. Meyer KJ, Chan C, Hopper L, Nicholson LL. Identifying lower limb specific and generalised joint hypermobility in adults: validation of the Lower Limb Assessment Score. BMC Musculoskelet Disord. 2017;18(1):514.

69. Nicholson LL, Chan C. The Upper Limb Hypermobility Assessment Tool: A novel validated measure of adult joint mobility. Musculoskelet Sci Pract. 2018;35:38-45.

70. Scheper MC, Nicholson LL, Adams RD, Tofts L, Pacey V. The natural history of children with joint hypermobility syndrome and Ehlers-Danlos hypermobility type: a longitudinal cohort study. Rheumatology (Oxford). 2017;56(12):2073-83.

71. Fikree A, Chelimsky G, Collins H, Kovacic K, Aziz Q. Gastrointestinal involvement in the Ehlers-Danlos syndromes. Am J Med Genet C Semin Med Genet. 2017;175(1):181-7.

72. Hakim A, O'Callaghan C, De Wandele I, Stiles L, Pocinki A, Rowe P. Cardiovascular autonomic dysfunction in Ehlers-Danlos syndrome-Hypermobile type. Am J Med Genet C Semin Med Genet. 2017;175(1): 168-74.

73. Claar RL, Simons LE, Logan DE. Parental response to children's pain: the moderating impact of children's emotional distress on symptoms and disability. Pain. 2008;138(1):172-9.

74. Palermo TM, Valrie CR, Karlson CW. Family and parent influences on pediatric chronic pain: a developmental perspective. Am Psychol. 2014;69(2):142-52.

75. Onghena P, Edgington ES. Customization of pain treatments: single-case design and analysis. Clin J Pain. 2005;21(1):56-68; discussion 9-72. 




\section{Summary}

Chronic musculoskeletal pain (CMP) is one of the most frequently reported pain complaints in adolescents and has a considerable disabling impact. In most cases, a specific somatic cause of CMP cannot be identified, and is defined as non-specific. Therefore, a biopsychosocial approach in the management of CMP is recommended and focussed on the complex interaction between physiological, psychological, and social factors. Two important factors which might contribute to understand the complexity and explain the disabling impact of CMP are pain-related fear and generalized joint hypermobility $(G J H)$. Therefore, the objective of this dissertation is to evaluate the specific influence of both pain-related fear and GJH on physical functioning in adolescents with CMP. GJH and CMP are also features of generalized hypermobility spectrum disorder (G-HSD) and hypermobile Ehlers-Danlos syndrome (hEDS), both symptomatic conditions without a specific genetic profile. Knowledge of these potential negative factors enables to further unravel the mechanism of disabling CMP in adolescents with G-HSD/hEDS and might lead to better tailored interventions.

Chapter 2, a topical review, describes the current state of evidence summarized and presented in an applied theoretical fear-avoidance model (FAM), which is a cognitivebehavioural framework, that proposes the role of GJH and pain-related fear as determinants for CMP-related disability in adolescents with G-HSD/hEDS. It is assumed that GJH seems to make adolescents more vulnerable for developing CMP due to an increased risk of (re)injury and a frequent experience of musculoskeletal pain. In addition, a vulnerability for pain-related fear and anxiety is proposed as core factor in the underlying mechanism explaining the level of disability in adolescents with G-HSD/hEDS. It is proposed that pain-related fear and anxiety cause avoidance behaviour. Long-term avoidance may lead to disuse, depression, and disability and eventually physical deconditioning, further fuelling the vicious circle of CMP. In adolescents with G-HSD/hEDS, specifically, physical deconditioning is hypothesized to have additional negative consequences. Their compensation mechanism, essential for joint stability to compensate for joint laxity, might fail resulting in impaired propriocepsis, balance, and body posture, which in turn might lead to an increased risk of injury and affect gait patterns. Therefore, in explaining disability, it is important to focus on both the negative physical components related to joint hypermobility, in tandem with the psychological components such as pain-related fear in adolescents with G-HSD/hEDS. Although scientific confirmation of this FAM applied to G-HSD/hEDS seems warranted, many studies are cross-sectional and therefore the suggested mechanism should be interpreted with caution. 
In chapter 3, we studied whether pain-related fear and joint hypermobility are related, and even reinforced each other, resulting in a higher level of disability in adolescents with CMP. In this cross-sectional study 116 adolescents with CMP participated, who were referred to the university outpatient rehabilitation clinic specialised in pain rehabilitation. The adolescents completed web-based questionnaires, which were part of regular health care procedure. The population was predominantly female with a mean age of 16.0 years and 40 (35\%) of them had joint hypermobility measured with the Beighton score. Based on a hierarchical regression analysis, with disability as the dependent variable, the hypothesis that heightened pain-related fear is a vulnerability leading to disability in hypermobile adolescents with CMP was tested. The results showed that only pain-related fear and perceived harmfulness, as a construct of painrelated fear, were associated with a higher level of disability in adolescents with CMP, despite being hypermobile or not. There were no differences in pain-related fear and perceived harmfulness between non-hypermobile and hypermobile adolescents with CMP. Having GJH probably makes an adolescent more vulnerable to developing chronic pain, but once an adolescent has chronic pain, especially pain-related fear is the factor associated with disability.

Earlier studies showed contradicting results of having asymptomatic GJH on physical functioning. Some studies reported lower levels of physical functioning, however other studies showed no differences in physical functioning. Therefore, in chapter 4 , the focus was on physical functioning (muscle strength, motor performance, and physical activity level) and the potential negative influence of pain-related fear on physical functioning in adolescents with asymptomatic GJH compared to non-hypermobile controls. Sixty-two healthy adolescents participated in this cross-sectional study. The mean age was 16.8 years, approximately $75 \%$ was female and $18 \%$ had asymptomatic GJH measured with the Beighton score. Muscle strength and endurance were measured with an isokinetic dynamometer (Biodex) and motor performance was assessed based on the single leg hop for distance. Physical activity measurement took place using a tri-axial accelerometer (AX3). The Photograph Series of Daily Activities for youth (PHODA-youth) measured perceived harmfulness, as a construct of pain-related fear. Hierarchical regression analyses were used to evaluate whether adolescents with asymptomatic GJH had decreased physical functioning levels compared to non-hypermobile adolescents. In addition, the potential negative role of pain-related fear was evaluated. The results indicated that adolescents with asymptomatic GJH had no lower physical functioning levels, regarding muscle strength and endurance, motor performance, and physical activity level compared to non-hypermobile controls. Results also showed that the level of perceived harmfulness was very low on all subscales related to the total score range 
in these pain-free adolescents and did not differ between the hypermobile and nonhypermobile adolescents.

Chapter 5 further focused on untangling the influence of having $\mathrm{GJH}$, having $\mathrm{CMP}$, and pain-related fear on physical functioning in adolescents. In this cross-sectional study four subgroups of adolescents were included: 21 adolescents with CMP and without GJH, 9 adolescents with both CMP and GJH, 51 adolescents without CMP and without GJH, and 11 adolescents without CMP and with GJH. The adolescents with CMP were recruited by a physician in rehabilitation medicine in the university outpatient rehabilitation clinic specialised in pain rehabilitation. The healthy adolescents without CMP were recruited in the same period as the adolescents with CMP. Therefore, the composition of the adolescent population without CMP was based on age and gender of the adolescents with CMP. As outcome variables: muscle strength and endurance (dynamometer), motor performance (single leg hop for distance), physical activity level (accelerometry), and pain-related fear (PHODA-youth) were assessed. Hierarchical regression analyses were used to study the level of physical functioning, as dependent variable, and the contribution of pain-related fear in adolescents with/without CMP as well as with/without GJH. Results showed that adolescents with CMP, despite having GJH or not, had decreased muscle strength and endurance and lower motor performance related to balance, coordination, and joint stability compared to pain-free adolescents (with/without GJH). There were no differences in PAL between adolescents with CMP (with/without GJH) and adolescents without CMP (with/without GJH). The results again showed that higher levels of pain-related fear, identified in adolescents with $\mathrm{CMP}$, were associated with decreased muscle strength and endurance and lower motor performance. It should be considered that an increased level of pain-related fear might also interfere with physical assessment itself. Adolescents with pain-related fear might decide to stop the assessment or perform submaximally, resulting in a lower score. In addition, the influence of pain-related fear on physical functioning did not differ between hypermobile and non-hypermobile adolescents with CMP. These results suggested that once adolescents have CMP, despite having GJH or not, the level of painrelated fear is the contributing factor to a lower level of physical functioning.

The results of a study using a pre-post-test design evaluating multidisciplinary rehabilitation treatment in 14 adolescents ( 1 male) with G-HSD/hEDS were presented in chapter 6 . The multidisciplinary rehabilitation treatment consisted out of a combination of physical training, which focused on improving aerobic capacity, muscle strength, and propiocepsis to compensate for the physical negative consequences, and a targeted cognitive-behavioural treatment; exposure in vivo, which focused on reducing pain- 
related fear. Results showed that adolescents with G-HSD/hEDS had significant and clinically relevant improvements (67\%) in the level of functional disability, which was the main outcome. Furthermore, significant post-treatment improvements were found in muscle strength and endurance, motor performance, perceived harmfulness, and pain intensity. The reduction in pain intensity (63\%) was remarkable, since pain reduction was not a goal of multidisciplinary rehabilitation treatment. Although the results of this multidisciplinary rehabilitation treatment in adolescents with G-HSD/hEDS are promising, our findings are based on a small sample size without a controlled group and therefore should be treated with caution.

In chapter 7, a general discussion on the main findings of this dissertation is provided. The studies in this dissertation focused on the role of having $\mathrm{GJH}$, having $\mathrm{CMP}$, and the potential influence of pain-related fear on physical functioning and disability within adolescents with G-HSD/hEDS. Therefore, knowledge of these elements contribute to further unravel the mechanism of disabling pain in adolescents with G-HSD/hEDS and might lead to better tailored interventions. Based on the results of this dissertation, it became clear that pain-related fear is an important determinant of diminished physical functioning and disability in adolescents with CMP, despite having GJH or not. Since, especially pain-related fear seems to be disabling, early detection of pain-related fear in primary care is warranted to identify adolescents who are at risk to further develop disabling CMP. In future tailored multidisciplinary rehabilitation treatment for adolescents with G-HSD/hEDS, who have considerable disability, it is important to focus especially on psychological components, such as pain-related fear, catastrophizing thoughts, and generalized anxiety to better enable these adolescents to optimally participate in society. Exposure in vivo might be the preferable intervention to restore the normal pattern of daily functioning by reducing pain-related fear. However, if decreased physical functioning components (such as muscle strength, cardiorespiratory fitness, and balance) are present which interferes with the capability of performing daily life activities, multidisciplinary rehabilitation treatment should contain a specific physical training focusing on improving the adverse physical components. Furthermore, the lower levels of physical functioning were associated with higher levels of pain-related fear in the adolescents with CMP. Pain-related fear might have influenced the objective measurements, since adolescents with CMP, despite having GJH or not, probably decided to stop or perform submaximally due to pain experience and/or pain-related fear. Therefore, in future research or clinical practice, when measuring physical functioning in adolescents with CMP, researchers or therapists should be aware of the psychological factors influencing the test outcome. 
In conclusion, this dissertation showed that pain-related fear is an important determinant of a decrease in physical functioning and disability in adolescents with CMP, despite being hypermobile or not. A multidisciplinary rehabilitation treatment of physical training and exposure in vivo seems promising in reducing pain-related disability in adolescents with G-HSD/hEDS. It is recommended to further develop the tailored multidisciplinary rehabilitation treatments incorporating psychological and physical elements when needed, to further enable these adolescents to optimally participate in society. 




\section{Samenvatting}

Het belangrijkste onderwerp van dit proefschrift is het ontrafelen van de rol van pijngerelateerde vrees en gegeneraliseerde gewrichtshypermobiliteit op het fysiek functioneren bij adolescenten met chronische musculoskeletale pijnklachten.

Hoofdstuk 1 begint met een algemene inleiding over chronische pijn, hypermobiliteit en pijn-gerelateerde vrees. Chronische pijn wordt gedefinieerd als pijn die langer dan 3 maanden aanhoudt. Wereldwijd lijdt 1 op de 5 volwassenen aan chronische pijn en wordt nog eens 1 op de 10 volwassenen jaarlijks met chronische pijn gediagnosticeerd. In de afgelopen jaren komt steeds meer aandacht voor chronische pijn bij kinderen en adolescenten. Naar schatting 25\% van de Nederlandse schoolkinderen ervaart een periode van chronische pijn. Gebaseerd op de locatie kan chronische pijn onderverdeeld worden in subgroepen. Naast buikpijn en hoofpijnklachten, is chronische musculoskeletale pijn (CMP) de meest voorkomende pijnklacht bij adolescenten. De incidentie van CMP neemt toe met de leeftijd en CMP komt vaker voor bij meisjes. In ongeveer $40 \%$ van de kinderen en adolescenten met CMP leidt pijn tot beperkingen in het dagelijks functioneren. CMP kan een negatieve invloed hebben op fysiek en psychologisch functioneren, kan leiden tot meer afwezigheid op school, kan interfereren met sociale activiteiten en familieaangelegenheden en kan resulteren in een afname van de kwaliteit van leven. Voor CMP kan vaak geen of geen afdoende medische verklaring worden gevonden en wordt dan geduid als aspecifiek. Psychosociale factoren spelen een belangrijke rol in het ervaren beperkingenniveau. Een biopsychosociale aanpak in de behandeling van CMP wordt dan ook aangeraden. Bij deze aanpak worden fysieke, psychologische en sociale factoren gebruikt om de complexe relatie tussen het ervaren van pijn en de impact op het dagelijks functioneren en participeren in de maatschappij te ontrafelen en hun impact te verkleinen. Twee belangrijke factoren die mogelijk bijdragen aan het begrijpen van de complexiteit en verklaren van de beperkingen gerelateerd aan CMP zijn pijn-gerelateerde vrees en gegeneraliseerde gewrichtshypermobiliteit (GJH). In chronische pijn bij kinderen en adolescenten is pijngerelateerde vrees een voorspeller van pijn-gerelateerde beperkingen in het dagelijks functioneren. Onderzoek toont aan dat gedurende adolescentie, GJH een risicofactor is voor het krijgen van CMP. GJH wordt gedefinieerd als een vergrote bewegingsuitslag in meerdere gewrichten. Voor veel individuen kan deze vergrote bewegingsuitslag voordelig werken bij het uitvoeren van diverse sporten zoals dansen en turnen. Afhankelijk van het type te bespelen instrumenten hebben musici eveneens profijt van GJH. Echter, GJH wordt ook gerelateerd aan CMP. GJH gerelateerd aan symptomen zoals CMP, gewrichtsinstabiliteit, verstoorde bewegingszin en weke delen letsels wordt betiteld als gegeneraliseerde hypermobiliteits spectrum aandoening (G-HSD). G-HSD 
heeft veel overlap met het hypermobiele subtype van Ehlers-Danlos syndroom (hEDS). G-HSD en hEDS zijn beide specifieke GJH-gerelateerde aandoeningen zonder een genetisch profiel, derhalve worden zij in dit proefschrift beschreven als G-HSD/hEDS. Adolescenten met G-HSD/hEDS hebben veel voorkomende fysieke symptomen zoals verminderde kracht, conditie, balans en verstoorde bewegingszin. Tevens is de mate van participatie in het dagelijks leven verlaagd. Daarnaast komen gegeneraliseerde angst en ook pijn-gerelateerde vrees vaker voor bij adolescenten met G-HSD/hEDS. Het huidige bewijs voor wetenschappelijke bewezen behandelingen voor adolescenten met G-HSD/hEDS is beperkt. Er worden veel verschillende behandelingen aangeboden met diverse elementen vaak zonder een duidelijk specifiek theoretisch concept.

Een hogere mate van pijn-gerelateerde vrees is een risico voor het krijgen van meer pijngerelateerde beperkingen op de lange termijn. Dit is eveneens veel voorkomend bij adolescenten met G-HSD/hEDS. Echter, de rol van pijn-gerelateerde vrees is onvoldoende belicht binnen de huidige behandelingen van deze patiëntengroep. Het vreesvermijdingsmodel is een theoretisch model waarin de beperkende rol van pijngerelateerde vrees wordt verklaard. Dit model is bewezen in zowel de volwassen, als de kind en adolescent populatie. Exposure in vivo is een cognitief gedragsmatige behandelvorm dat zich specifiek richt op deze pijn-gerelateerde vrees. Bij adolescenten en volwassenen is exposure in vivo effectief in het verminderen van beperkingen. Mogelijk zou exposure in vivo ook toegepast kunnen worden bij adolescenten met G-HSD/hEDS om hun functioneren te optimaliseren.

De nadruk in dit proefschrift ligt dan ook op het verder ontrafelen van de individuele rol van pijn-gerelateerde vrees en GJH op het fysiek functioneren bij adolescenten met CMP. Het beter begrijpen van deze onderlinge relaties zou mogelijk kunnen leiden tot betere gepersonaliseerde behandelingen.

In hoofdstuk 2 wordt de huidige wetenschappelijke literatuur met betrekking tot het fysiek en psychologisch functioneren van adolescenten met GJH en G-HSD/hEDS samengevat en gepresenteerd in een toegepast vreesvermijdingsmodel, waarin voorgesteld wordt dat GJH en pijn-gerelateerde vrees belangrijke factoren zijn voor CMP-gerelateerde beperkingen. Een onderliggende aanname in het model is, dat GJH adolescenten kwetsbaarder maakt voor het ontwikkelen van CMP, door een verhoogd risico op het krijgen van blessures en het vaker ervaren van pijnklachten. Daarnaast worden pijn-gerelateerde vrees en meer gegeneraliseerde angst verondersteld als belangrijkste onderliggend factoren in het verklaren van beperkingen bij adolescenten met G-HSD/hEDS. Volgens het model leidt pijn-gerelateerde vrees tot vermijdingsgedrag en uiteindelijk tot beperkingen in het dagelijks functioneren, depressie en op de lange termijn tot fysieke deconditionering. Deze factoren zijn weer van invloed op het in stand 
houden van de vicieuze cirkel van CMP. In adolescenten met G-HSD/hEDS wordt in het aangepaste vreesvermijdingsmodel verondersteld dat met name de fysieke deconditionering leidt tot additionele negatieve consequenties. Hun compensatiemechanisme gebaseerd op spierkracht, dat essentieel is voor gewrichtsstabiliteit om te compenseren voor de GJH, verslechtert door fysieke deconditionering en leidt tot verminderde bewegingsgevoel, balans en lichaamshouding. Dit beïnvloedt mogelijk het gangpatroon en zou kunnen leiden tot een verhoogde kans op blessures. Derhalve, in het verklaren van beperkingen, is het belangrijk om zowel op de negatieve fysieke componenten gerelateerd aan GJH, als op de psychologische factoren zoals pijngerelateerde vrees de nadruk te leggen bij adolescenten met G-HSD/hEDS. Hoewel het vreesvermijdingsmodel toegepast op adolescenten met G-HSD/hEDS door wetenschappelijke literatuur onderbouwd wordt, zijn veel onderzoeken transversaal en daarom moet het voorgestelde mechanisme met de nodige voorzichtigheid geïnterpreteerd worden.

Hoofdstuk 3 onderzoekt of pijn-gerelateerde vrees en GJH samenhangen en elkaar zelfs versterken met als gevolg meer beperkingen in het dagelijks functioneren bij adolescenten met CMP. In deze cross-sectionele studie namen 116 adolescenten met CMP deel, die verwezen waren naar de gespecialiseerde revalidatiepolikliniek op het gebied van pijn in het universitaire ziekenhuis van Maastricht/Adelante Zorggroep. De adolescenten vulden online vragenlijsten in, die onderdeel waren van de reguliere zorg. De populatie bestond voornamelijk uit meisjes met een gemiddelde leeftijd van 16.0 jaar en 40 (35\%) voldeden aan criteria voor GJH gemeten met de Beighton score. Middels hiërarchische regressiemodellen, met beperkingen in het dagelijks functioneren als afhankelijke variabele, is de hypothese getoetst of pijn-gerelateerde vrees en ervaren schadelijkheid als construct van pijn-gerelateerde vrees, samenhangt met meer beperkingen bij hypermobiele adolescenten met CMP. De resultaten lieten zien dat pijngerelateerde vrees en ervaren schadelijkheid gerelateerd waren aan meer beperkingen, onafhankelijk van wel of niet hypermobiel zijn. Er waren geen verschillen tussen de mate van pijn-gerelateerde vrees en ervaren schadelijkheid tussen adolescenten met CMP die hypermobiel waren en adolescenten met CMP die niet hypermobiel waren. Het hebben van GJH maakt de adolescent mogelijk kwetsbaarder voor het krijgen van CMP. Echter, op het moment dat sprake is van CMP, is pijn-gerelateerde vrees gerelateerd aan beperkingen in het dagelijks functioneren en niet het wel of niet hebben van hypermobiliteit.

Eerdere studies lieten tegenstrijdige resultaten zien met betrekking tot het hebben van asymptomatische GJH, ofwel GJH zonder pijnklachten, op het fysiek functioneren. 
Sommige studies rapporteerden een lagere niveau van fysiek functioneren, terwijl andere studies geen verschil vonden in het niveau van fysiek functioneren van pijnvrije adolescenten met GJH en pijnvrije adolescenten zonder GJH. Derhalve was de focus in hoofdstuk 4 op het onderzoeken van het niveau van fysiek functioneren (spierkracht, bewegingscoördinatie en fysiek activiteitenniveau) van asymptomatische adolescenten met GJH in vergelijking met dat van niet-hypermobiele adolescenten. Verder werd geëvalueerd of een hogere mate van pijn-gerelateerde vrees samenhing met een mogelijk lager niveau van fysiek functioneren. In deze cross-sectionele studie namen 62 gezonde adolescenten deel. De gemiddelde leeftijd was 16.8 jaar, ongeveer $75 \%$ was vrouwelijk en $18 \%$ had asymptomatische GJH gemeten met de Beighton score. Spierkracht en krachtuithoudingsvermogen werden gemeten met een isokinetische dynamometer (Biodex) en bewegingscoördinatie werd bepaald middels de single leg hop for distance. Het fysiek activiteitenniveau werd gemeten middels tri-axiale accelerometrie (AX3). Voor het in kaart brengen van de ervaren schadelijkheid, als construct van pijn-gerelateerde vrees, werd de the Photograph Series of Daily Activities for youth (PHODA-youth) afgenomen. In de analyse werden hiërarchische regressiemodellen gebruikt om te evalueren of adolescenten met asymptomatische GJH een lagere niveau van fysiek functioneren hebben vergeleken met niet-hypermobiele adolescenten. Daarnaast, werd de mogelijke negatieve rol van pijn-gerelateerde vrees geëvalueerd. De resultaten lieten zien dat adolescenten met asymptomatische GJH geen lager niveau van fysiek functioneren, met betrekking tot spierkracht, bewegingscoördinatie en fysiek activiteitenniveau, hadden in vergelijking met niet-hypermobiele adolescenten. Tevens was de gerapporteerde ervaren schadelijkheid zeer laag op alle subschalen bij deze pijnvrije adolescenten en werden ook geen onderlinge verschillen gemeten tussen adolescenten met GJH en niet-hypermobiele adolescenten.

De focus in hoofdstuk 5 ligt op het verder ontrafelen van de invloed van het hebben van alleen CMP, het hebben van alleen GJH, het hebben van GJH en CMP, en de rol van pijngerelateerde vrees op het fysiek functioneren bij adolescenten. In deze cross-sectionele studie werden 4 subgroepen van adolescenten geïncludeerd: 30 adolescenten met CMP (waarvan 21 zonder GJH en 9 met GJH) en 62 adolescenten zonder CMP (waarvan 51 zonder GJH en 11 met GJH). De adolescenten met CMP werden gerekruteerd door een revalidatiearts op de polikliniek van Adelante Zorggroep, locatie Maastricht Universitair Medisch Centrum+. De gezonde adolescenten werden geïncludeerd in dezelfde tijdsperiode. De groepssamenstelling van de adolescenten zonder CMP is afgestemd op leeftijd en geslacht van de adolescenten met CMP. De uitkomstvariabelen om fysiek functioneren te meten waren spierkracht en krachtuithoudingsvermogen (dynamometer), bewegingscoördinatie (single leg hop for distance) en fysiek 
activiteitenniveau (accelerometrie). Pijn-gerelateerde vrees werd gemeten met de PHODA-youth. In de analyse, werden hiërarchische regressiemodellen gebruikt om zowel het niveau van fysiek functioneren, als afhankelijke variabele, als ook de bijdrage van pijn-gerelateerde vrees in adolescenten met/zonder CMP en met/zonder GJH te bestuderen. De resultaten lieten zien dat adolescenten met CMP, onafhankelijk van wel of niet hebben van hypermobiliteit, verminderde spierkracht, krachtuithoudingsvermogen en bewegingscoördinatie hadden in vergelijking met pijnvrije adolescenten (met/zonder GJH). Het fysiek activiteitenniveau verschilden niet tussen adolescenten met CMP (met/zonder GJH) vergeleken met adolescenten zonder CMP (met/zonder GJH). De resultaten toonden opnieuw aan dat een hogere mate van pijn-gerelateerde vrees, geïdentificeerd in adolescenten met CMP, gerelateerd was aan verminderde spierkracht, krachtuithoudingsvermogen en bewegingscoördinatie. Mogelijk dat een verhoogde mate van pijn-gerelateerde vrees eveneens van invloed was op de afname van de fysieke testen. Adolescenten met pijn-gerelateerde vrees besloten mogelijk om te stoppen of niet een maximale prestatie te leveren, wat resulteerde in een lagere score. De invloed van pijn-gerelateerde vrees op het fysiek functioneren verschilde niet tussen hypermobiele en niet-hypermobiele adolescenten met CMP. Deze resultaten suggereerden dat: als adolescenten eenmaal CMP hebben, onafhankelijk van wel of niet hypermobiel zijn, de mate van pijn-gerelateerde vrees de meest bijdragende factor is aan het verminderd fysiek functioneren.

Hoofdstuk 6 presenteert de resultaten van een studie in een pre-post-test design gericht op het evalueren van de verandering in beperkingen, fysiek functioneren, ervaren schadelijkheid en pijn intensiteit na deelname aan een multidisciplinaire revalidatiebehandeling van adolescenten met G-HSD/hEDS. De multidisciplinaire revalidatiebehandeling bestond uit een combinatie van fysieke training en een gerichte cognitieve gedragsmatige therapie. De fysieke training was gericht op het verbeteren van de aerobe capaciteit, spierkracht en bewegingsgevoel met als doel te compenseren voor de fysieke negatieve consequenties. De cognitieve gedragsmatige therapie was exposure in vivo en had als doel het reduceren van pijn-gerelateerde vrees. Veertien adolescenten (1 man) werden geïncludeerd. Uit de resultaten bleek dat de adolescenten met G-HSD/hEDS een significante en klinische relevante vooruitgang (67\%) boekten op de ervaren mate van beperkingen in het dagelijks functioneren, welke de belangrijkste uitkomstmaat was. Tevens werden na de multidisciplinaire revalidatiebehandeling significante verbeteringen gevonden voor spierkracht, krachtuithoudingsvermogen, bewegingscoördinatie, ervaren schadelijkheid en pijn intensiteit. Met name de afname van pijn intensiteit (63\%) was opvallend, aangezien pijnreductie niet een doel was van de multidisciplinaire revalidatiebehandeling. De resultaten van deze multidisciplinaire 
revalidatiebehandeling voor adolescenten met G-HSD/hEDS zijn veelbelovend. Echter deze bevindingen dienen met de nodige voorzichtigheid geïnterpreteerd te worden, aangezien de resultaten gebaseerd zijn op een vergelijking van het functioneren voor en na de behandeling van een kleine groep zonder controlegroep.

In hoofdstuk 7 wordt de algehele discussie gepresenteerd met een bespreking van de belangrijkste bevindingen van dit proefschrift. In de studies uitgevoerd en beschreven in dit proefschrift ligt de nadruk op de rol van GJH, CMP en de mogelijke invloed van pijngerelateerde vrees op het fysiek functioneren en beperkingen in het dagelijks functioneren bij adolescenten met G-HSD/hEDS. Daardoor draagt kennis van deze elementen bij aan het verder ontrafelen van het mechanisme van invaliderende pijn bij adolescenten met G-HSD/hEDS en leidt dit mogelijk ook tot betere op maat gemaakte behandelingen. Gebaseerd op de resultaten van dit proefschrift werd duidelijk dat pijngerelateerde vrees een belangrijke determinant is voor het verminderd fysiek functioneren en de ervaren beperkingen in het dagelijks functioneren bij adolescenten met CMP, onafhankelijk van het wel of niet hypermobiel zijn. Omdat met name pijngerelateerde vrees leidt tot beperkingen, is het vroegtijdig herkennen van pijngerelateerde vrees in de eerstelijnszorg belangrijk om adolescenten te identificeren die het risico lopen om nog meer beperkt te raken ten gevolge van CMP. In toekomstige op maat gemaakte multidisciplinaire revalidatiebehandelingen voor adolescenten met G-HSD/hEDS, die aanzienlijke beperkingen ervaren, is het belangrijk om naast fysieke componenten specifiek te focussen op psychologische factoren, zoals pijn-gerelateerde vrees, catastroferende gedachtes en gegeneraliseerde angst om deze adolescenten te faciliteren om optimaal te functioneren in de maatschappij. Exposure in vivo zou mogelijk de wenselijke behandeling zijn gericht op het herstellen van een normaal dagdagelijks functioneren middels het reduceren van pijn-gerelateerde vrees. Echter, mocht er eveneens sprake zijn van verminderd fysiek functioneren (zoals spierkracht, aerobe capaciteit en balans) wat interfereert met de mogelijkheid om dagdagelijkse activiteiten uit te voeren, dan dient de multidisciplinaire revalidatiebehandeling eveneens een specifieke fysieke training gericht op het verbeteren van deze negatieve fysieke componenten te bevatten. Bovendien, de gevonden lagere niveaus van fysiek functioneren waren gerelateerd aan een hogere mate van pijn-gerelateerde vrees in adolescenten met CMP. Pijn-gerelateerde vrees heeft mogelijk de objectieve fysieke metingen beïnvloed bij adolescenten met CMP, onafhankelijk van wel of niet hypermobiel zijn. Het ervaren van pijn of pijn-gerelateerde vrees tijdens de meting heeft mogelijk geleid tot eerder stoppen van de test of niet maximaal inspannen tijdens de test. Daarom is het belangrijk, in toekomstig onderzoek en in de klinische praktijk, dat 
onderzoekers en therapeuten alert zijn op de invloed van psychologische factoren op de fysieke uitkomstmaat bij adolescenten met CMP.

Concluderend, dit proefschrift toont aan dat pijn-gerelateerde vrees een belangrijke determinant is van verminderd fysiek functioneren en beperkingen in het dagelijks functioneren bij adolescenten met CMP, onafhankelijk van wel of niet hypermobiel zijn. Een multidisciplinaire revalidatiebehandeling van fysieke training en exposure in vivo lijkt veelbelovend in het verminderen van pijn-gerelateerde beperkingen bij adolescenten met G-HSD/hEDS. Het is aanbevolen om op maat gemaakte multidisciplinaire revalidatiebehandelingen met psychologische elementen en indien nodig fysieke elementen verder te ontwikkelen, zodat deze adolescenten beter in staat zijn om optimaal te kunnen functioneren in de maatschappij. 




\section{Impact paragraph}

Besides a contribution to scientific knowledge, the results of this dissertation have a broader societal impact. This impact paragraph aims to describe the societal impact of the scientific results to application and implementation in the healthcare, to whom our findings are relevant, and activities for knowledge transfer.

\section{Relevance}

Each year, health care costs for patients with chronic musculoskeletal pain (CMP) are rising and many patients with CMP perceived their treatment as inadequate. In many patients with CMP, pain has a disabling impact. In adolescents, CMP may have a negative impact on physical and psychological functioning, interferes with school attendance, performance in social activities, and family engagement, leading to a reduced quality of life. The presence of pain-related fear and generalized joint hypermobility might contribute to understand the complexity and explain the disabling impact of CMP in adolescents. Better understanding of the mechanism may stimulate the development of personalised treatments for hypermobile adolescents with CMP.

Studies in this dissertation showed that pain-related fear is associated with higher levels of disability and lower physical functioning in adolescents with CMP, despite being hypermobile or not. Therefore, early detection of pain-related fear in primary care is important to identify adolescents with musculoskeletal pain who are more at risk for developing disabling CMP. Health care professionals in primary care should thoroughly assess the adolescent's pain problem and needs, make use of biopsychosocial measurements, and be aware of generalized joint hypermobility in order to refer the patient to the right treatment at the right moment. In case of minimal disability in daily life activities with only little influence of psychosocial factors, health care professionals in primary care should use a biopsychosocial approach to get adolescents with CMP active and restore age appropriate functioning despite pain. However, for the adolescents with CMP, despite being hypermobile or not, with a moderate to high level of disability in daily life activities and associated influence of psychosocial factors, multidisciplinary rehabilitation treatment in primary or secondary care are recommended.

The intervention presented in this dissertation, consisted out of a multidisciplinary rehabilitation treatment with physical training and a targeted cognitive-behavioural therapy intervention; exposure in vivo. Results of the study showed a decrease in the level of disability, improvements in physical functioning, and reduced level of painrelated fear and pain intensity in hypermobile adolescents with CMP. It is recommended to further develop tailored multidisciplinary rehabilitation treatments for hypermobile adolescents with CMP incorporating psychological and physical elements when needed. 
Physical elements should be added to the multidisciplinary rehabilitation treatment when objective physical measurements show a decrease in physical functioning, such as muscle strength, balance, and aerobic capacity, which interferes with the capability of performing daily life activities. It should be noted that during the assessment of physical functioning, pain experience and psychological factors such as pain-related fear may influence the assessment, and thus the outcome. Adolescents with CMP might decide to stop or perform submaximally, resulting in a lower score. Health care professionals or researchers should be aware of that. Combining different measurements with the same physical elements outcome, for instance measuring muscle strength isokinetic and functional, provides a broader view of the adolescent's physical functioning and differentiates discrepancies in the outcome measures.

\section{Target group}

Hypermobile adolescents with CMP might benefit from the multidisciplinary rehabilitation treatment presented in this dissertation. The combination of physical training and exposure in vivo led to a significantly and clinically relevant reduction in the level of disability. In addition, physical functioning, pain-related fear, and pain intensity improved significantly.

Furthermore, the parents and other family members of adolescents with CMP could benefit from the findings of this dissertation. Previous studies showed that parents and families might have a negative influence on the adolescent's pain, pain behaviour, and disability. Parents and families of adolescents with CMP have more social restrictions, less family functioning (such as more conflict or less cohesion), and higher levels of parental stress, anger, depression, and anxiety. Parents also experience the financial burden of CMP. Improvements of the adolescent's functioning benefits the parents and families as well. The negative impact on parental stress, anger, depression, social restriction, and family functioning might decrease.

The studies presented in this dissertation might be of interest for researchers who focus on unravelling the concept of disability in hypermobile adolescents with CMP. Especially, the focus of this dissertation on the impact of pain-related fear on disability in hypermobile adolescents with CMP is a novel topic and is relevant for researchers aiming to improve personalised and tailored treatments for this group of patients. The results of this dissertation contribute to the existing literature of a multidisciplinary and patient-centred approach in hypermobile adolescents with CMP. There is limited evidence for the effect of the often applied more passive approach, such as pain medication, use of mobility aids, and splinting for hands or wrists in restoring age appropriate functioning in hypermobility-related pain. Therefore, an active 
biopsychosocial approach in hypermobile adolescents with CMP is recommended to restore age appropriate functioning in society despite having pain.

Furthermore, health care professionals who treat adolescents with CMP in their clinical practice could benefit from this dissertation. Recognizing and treating pain-related fear or recognizing pain-related fear and referral to specialised care adds to delivering the right care at the right moment and reducing the burden of CMP in adolescents and their parents and family. In addition, health care professionals in primary care, for instance a school physician, should be aware of generalized joint hypermobility in the general painfree population. Generalized joint hypermobility might be a risk factor for developing $\mathrm{CMP}$, especially if these adolescents also experiencing pain-related fear.

Finally, the results presented are of interest for health care insurance companies. Effective and tailored treatments and referral of hypermobile adolescents with CMP to the right place might reduce the financial burden of unnecessary costs, such as medical shopping or overtreatment of inadequate therapies. Therefore, more insight in the costeffectiveness of the multidisciplinary rehabilitation treatment for hypermobile adolescents with CMP compared to the usual rehabilitation treatment is needed.

\section{Knowledge transfer}

This dissertation shows the promising results of multidisciplinary rehabilitation treatment in hypermobile adolescents with CMP consisting of physical training and exposure in vivo. New studies with more rigorous research designs, such as a single-case design or randomized controlled trial, are warranted to further investigate and confirm clinical effectiveness of this multidisciplinary rehabilitation treatment. In case of proven effectiveness, this treatment can be included in Dutch and international guidelines. In addition, in order to raise awareness of the promising results of this new multidisciplinary rehabilitation treatment for hypermobile adolescents with CMP, national and international patient organisations could share this information on their media channels. To disseminate the scientific results of this dissertation, workshops have been organized and presentations on national and international conferences were given for researchers and health care professionals. A course has already been developed (provided at the Adelante Academy) aimed at the training of multidisciplinary rehabilitation teams to use the exposure in vivo protocol in adolescents with CMP. Furthermore, there is an interdisciplinary course for health care professionals in primary care (provided at the Adelante Academy) for using a common biopsychosocial approach in $\mathrm{CMP}$, recognizing pain-related fear, and decide adequately when to refer to specialised care. Health care professionals could also read the book about exposure, which is written by our researchers and clinicians and published in the Dutch language. This book 'Graded Exposure, een cognitief gedragsmatige aanpak van chronische pijn' 
describes the application of exposure in both adolescents and adults with different chronic pain conditions. The results from our studies are published in international scientific journals. 



\section{Dankwoord}

Eindelijk mag ik starten met het schrijven van het laatste hoofdstuk van mijn proefschrift; het dankwoord. Sommigen vinden dit het belangrijkste hoofdstuk van een proefschrift en meestal wordt dit hoofdstuk ook het meest gelezen. Ik persoonlijk denk dat de eerdere hoofdstukken ook zeker de moeite zijn om te lezen en dat deze dan ook een mooie afspiegeling zijn van mijn harde werk en met mij nog vele anderen. Daarom wil ik in dit laatste hoofdstuk, naast mijn collega's van de vakgroep revalidatiegeneeskunde UM, collega's van het Adelante kenniscentrum en collega's van de afdeling revalidatiegeneeskunde Adelante, locatie MUMC+ een aantal personen nadrukkelijk bedanken die mij de laatste 6 jaar geholpen hebben om naast mijn werk als fysiotherapeut dit promotietraject af te kunnen ronden. Ik realiseer mij, dat ik ook mogelijk mensen zal vergeten die toch een rol gespeeld hebben. Mocht dit zo zijn, alsnog hartelijk bedankt.

Allereerst wil ik mijn promotieteam prof. dr. Jeanine Verbunt, prof. dr. Raoul Engelbert en dr. Ivan Huijnen bedanken die het meest bijgedragen hebben aan dit proefschrift.

Beste Jeanine, als eerste promotor was jij vanaf het begin af aan betrokken mijn traject. Ik leerde jou kennen als revalidatiearts op onze afdeling en samen ontwikkelden wij de combinatiemodule van fysieke training en exposure voor hypermobiele adolescenten met chronische musculoskeletale pijn. Jij was ook degene die mij als eerste benaderde voor een junior onderzoeker functie op de afdeling revalidatiegeneeskunde aan de UM. Door de jaren heen heb ik jou leren kennen als een betrouwbare en gedreven begeleider. Ondanks dat jouw agenda steeds voller raakte en jij steeds meer nevenfuncties kreeg, bleef jij altijd kritische feedback leveren aan mijn stukken. Ik wil jou heel hartelijk danken voor alle energie en tijd die jij in mij gestoken hebt en hopelijk kunnen wij in de toekomst blijven samenwerken.

Beste Raoul, als tweede promotor sloot jij later aan bij het promotieteam als specialist op het onderwerp gegeneraliseerde gewrichtshypermobiliteit. Ik zal één van onze eerste ontmoetingen nooit vergeten, jij wist mij die dag op de wetenschapsdag WCF van het KNGF meteen te prikkelen met de vraag of ik echt wilde parttime promoveren naast mijn reguliere fysiotherapiewerkzaamheden. In de afgelopen jaren was jouw feedback altijd razendsnel, opbouwend en eerlijk. Jouw positieve manier van benadering maakte alleen maar dat ik nog harder ging werken om mijn proefschrift af te ronden. Raoul, enorm bedankt, en hopelijk kunnen wij in de toekomst voortborduren op onze prettige samenwerking.

Beste Ivan, als copromotor en directe begeleider was jij altijd mijn eerste aanspreekpunt. Ik moet jou misschien nog wel het meeste van iedereen bedanken. Jij was het 
uiteindelijk die mij alsnog liet uitnodigen voor de vacature van fysiotherapeut op de afdeling revalidatiegeneeskunde Adelante, locatie MUMC+. Aan het begin van mijn carrière als fysiotherapeut was jij mijn directe collega. Daarna werd jij mijn begeleider als junior onderzoeker en uiteindelijk was jij mijn copromotor en directe begeleider binnen het promotietraject. Gedurende al die jaren kon ik altijd bij jou terecht, zeker in het begin van mijn traject waarin het leren loslaten en reële doelen stellen nog een aandachtspunt was. Ook jij had een steeds drukker wordende agenda, maar desalniettemin maakte jij altijd tijd, zelfs als de voetbalanalyses ten sprake kwamen. Jouw feedback was eveneens altijd opbouwend en kritisch en maakten mijn stukken door de jaren heen steeds beter. Ivan, enorm bedankt voor alles, ik heb veel van jou geleerd.

De leden van de beoordelingscommissie, Prof. dr. Rob Smeets (voorzitter), Prof. dr. Rob de Bie, Prof. dr. Annemieke Buizer, dr. Lies Rombaut en Prof. dr. Johan Vlaeyen, wil ik graag bedanken voor het lezen en beoordelen van dit proefschrift.

In de verschillende studies van dit proefschrift hebben meerdere adolescenten met en zonder chronische musculoskeletale pijn deelgenomen. Zonder hun bereidheid om een bijdrage te leveren aan medisch wetenschappelijk onderzoek was dit proefschrift er niet geweest. Dank voor jullie deelname.

Arnoud Conijn, ik weet nog goed dat ik jou benaderde als studentonderzoeker. Jij had je via Jeanine Verbunt aangemeld en een eerdere mail van een collega van de vakgroep was jou ontschoten. Wat was ik daar blij om, gedurende de jaren heb jij mij enorm geholpen. Eerst als studentonderzoeker en later ook als WESP-student. Uiteindelijk heeft dit tot twee mooie publicaties geleid en is jouw interesse in de revalidatiegeneeskunde dusdanig gegroeid dat jij misschien nog wel gaat specialiseren tot revalidatiearts, ook al is dat in Rotterdam. Arnoud, hartstikke bedankt voor jouw bijdrage aan dit proefschrift.

Verder wil ik alle andere coauteurs bedanken voor hun bijdrage aan mijn publicaties: Laura Simons, Carlijn Wiertz, en Nicole Stappers.

Margareth van Krevel, als secretaresse van de afdeling revalidatiegeneeskunde van de UM heb jij vaak organisatorische zaken voor mij geregeld. Onze koffiemomentjes op dinsdagochtend zal ik gaan missen. Bedankt voor al jouw hulp. 
Inge Timmers, onder andere jouw Excel en Endnote kennis hebben mij vele uren ploeteren bespaard. Tevens heb ik jouw hulp tijdens het ISPP in Basel als zeer prettig ervaren. Bedankt hiervoor.

Albére Köke, "de wandelende encyclopedie", gedurende mijn traject heb jij mij meermaals geholpen met verschillende zaken zoals extra informatie over psychometrische eigenschappen van vragenlijsten of de rol van het autonome zenuwstelsel op de pijnervaring. Tijdens onze gezamenlijke trip naar India leerde ik jou goed kennen en jouw open kritische denkwijze fascineert mij. Ik hoop in de toekomst nog vaker met jou samen te werken.

Marlies den Hollander, jij weet als geen ander hoe het is om de combinatierol van promovendus en therapeut te hebben. Gedurende mijn traject was jij altijd oprecht geïnteresseerd in mijn vorderingen en heb jij mij ook meermaals geholpen bij het goed verwoorden van de psychologische elementen in dit proefschrift. Daarnaast hebben wij samen een lezing op een nationaal congres gegeven en al meermaals cursussen Exposure aangeboden. Jouw theoretische kennis en toewijding voor exposure zijn inspirerend. Dank voor al jouw ondersteuning.

Marion de Mooij, als onderzoeksassistent van 2B Active heb ik meermaals gebruik gemaakt van jouw hulp, bedankt hiervoor.

Richard Geers, als medisch technoloog was jij degene die de algoritmes voor mij gemaakt heeft voor het analyseren van de ruwe data van de accelerometers. Dank hiervoor.

Reni van Erp, gedurende jouw eigen promotie heb jij meermaals de fysieke metingen uitgevoerd bij de adolescenten met chronische musculoskeletale pijn. Dankjewel.

Hans Veders, hoe combineer je wetenschappelijk onderzoek, onderwijs en reguliere zorg? Een planner zoals jij maakt dit allemaal mogelijk. Door de jaren heen bouwde jij mijn planning flexibel om al mijn afspraken heen en werden de fysieke metingen bij hypermobiele adolescenten met chronische musculoskeletale pijn onderdeel van de reguliere zorg. Daarnaast leerde ik gaandeweg ook beter te organiseren en plannen, mede op basis van onze gesprekken. Dank voor al jouw hulp, zeker ook voor alle gezelligheid en samen zullen wij dit gaan vieren, na zdrowie. 
Ben Ummels, als fysiotherapie assistent was jij direct betrokken bij de trainingsmodule van hypermobiele adolescenten met chronische musculoskeletale pijn. Jouw enthousiaste en gedreven uitstraling binnen de behandeling hebben zeker bijgedragen aan de positieve behandelresultaten. Daarnaast, heb jij mij veelvuldig ondersteund bij de fysieke metingen van de adolescenten zonder pijnklachten en heb jij ook meermaals patiënten van mij overgenomen zodat ik tijd vrij had voor onderzoekstaken. Benny, "opa", bedankt voor al jouw tijd en energie en geniet de komende jaren van jullie prachtige project in Thailand.

Christoph Loo, jij was als manager eveneens betrokken bij mijn sollicitatieprocedure en gelukkig mocht ik ook nog blijven. Door de jaren heen heb jij mij altijd de ruimte gegeven om naast mijn zorgtaken te kunnen promoveren. Dank hiervoor.

Dennis Linden, als directe collega van de fysiotherapie was jij ook meermaals betrokken bij de fysieke metingen van de adolescenten met chronische musculoskeletale pijn. Daarnaast was jij vaak als fysiotherapeut betrokken bij de hypermobiliteitsmodule. Dankjewel.

Debby van Menxel en Emmelien Spek, ik mocht van jullie aansluiten bij het jongerenteam en samen hebben wij de afgelopen jaren de exposure behandeling van jongeren met pijn verder vormgegeven. Tevens hebben wij de cursus exposure bij jongeren ontwikkeld. Debby met jou heb ik ook nog meerdere symposia door het land gegeven en getracht de exposure boodschap door het land te verspreiden. Onze maandagochtend jongerenscreening was toch altijd de perfecte start van de werkweek. Bedankt, ook voor alle leuke momenten.

Angelo en Irene, als schoonouders waren jullie ook nauw betrokken bij mijn promotietraject. Gedurende de afgelopen jaren toonden jullie veel interesse in mijn werk en worden de hard copies van mijn publicaties goed bewaard door jullie. Tevens hebben jullie meermaals op Lize opgepast zodat ik mijn werkzaamheden op dinsdagmiddag voor de UM kon blijven continueren. Ik wil jullie dan ook bedanken voor jullie interesse en tijd.

Pap en mam, jullie hebben er mede voor gezorgd dat ik dit proefschrift heb kunnen afronden. Jullie maakte het mogelijk om na 4 jaar studeren aan de UM alsnog de opleiding fysiotherapie te gaan volgen. Hierdoor kon ik mij verder ontwikkelen en kijk waar wij nu staan. Jullie hebben mij altijd gestimuleerd om mijn dromen na te leven. Bedankt voor alles, zonder jullie was dit alles niet mogelijk geweest. 
Mijn paranifmen, Luuk van Meulenbroek en Kayl Pijpers.

Luuk, als tweelingbroer zijn wij vanaf dag 1 van ons leven samen opgegroeid. Qua uiterlijk verschillend, qua karakter zoveel hetzelfde. Door de jaren heen ben jij meer dan een broer voor mij geworden en ik ben dan ook trots dat jij straks aan mijn zijde zult staan.

Kayl, ik leerde jou kennen op de opleiding fysiotherapie. Beide hadden wij al een studie gedaan en vanaf het eerste moment konden wij het samen goed vinden. Door de jaren heen werden wij goede vrienden en hebben wij menig gezellige avond doorgebracht. Ik heb jou mogen helpen met jouw masterscriptie fysiotherapie en jij toonde altijd veel interesse in mijn promotietraject. Fantastisch dat wij dit straks samen kunnen meemaken en vieren.

En als allerlaatste mijn eigen gezin. Iverna, jij hebt mij de afgelopen jaren alle vrijheid gegeven om te kunnen promoveren. Als ik in de avonduren of weekenden besloot om nog extra tijd aan mijn promotie te besteden was dat nooit een probleem. In de beginfase van mijn promotie heb jij mij ook meermaals geholpen hoe om te gaan met eigen gecreëerde werkdruk. Lize, jouw geboorte 3 jaar geleden was het mooiste wat jouw mama en papa overkomen is. Voor mij was jij ook de ideale afschakeling na een drukke dag werken. Door met jou te spelen en te knuffelen kwam ik altijd tot rust en jij was mijn natuurlijke rem op mijn werkzaamheden. Dames, zonder jullie was dit niet gelukt. Ik ben trots op jullie en hou van jullie. 




\section{About the author}

Thijs van Meulenbroek was born on the $11^{\text {th }}$ of February 1986 in Heerlen, the Netherlands. He finished secondary school education at Groenewald in Stein. In 2004, he started the Bachelor of Health Sciences at Maastricht University. After the bachelor, Thijs continued his education at Maastricht University with the Master Physical Activity and Health, which was a specialisation of Human Movement Sciences. He obtained his Master's degree in 2008. In September 2008, he started to work as a tutor and trainer in the different courses of the Bachelor of Health Sciences at Maastricht University. His interest in the different pathologies and specifically the opportunity to treat these people made him choose to continue studying and started the study Physiotherapy at Zuyd University of Applied Sciences in Heerlen in 2009. Thijs obtained his Bachelor degree of Physiotherapy with distinction in 2012. After graduation, he started to work as a physiotherapist in the outpatient rehabilitation team of Adelante Zorggroep/ Maastricht University Medical Center+. In his clinical work as physiotherapist he focuses on pain rehabilitation in adolescents and adults, as well as rehabilitation in neurologic and orthopaedic patients. In October 2014, he was first appointed as a part time junior researcher (0.2 FTE) at the department of Rehabilitation Medicine at Maastricht University and from August 2016 as part time PhD-student (0.2 FTE) on the project "The impact of pain-related fear and hypermobility on physical functioning in adolescents with chronic musculoskeletal pain". He was supervised by Prof. dr. Jeanine Verbunt, Prof. dr. Raoul Engelbert, and dr. Ivan Huijnen. Throughout his project, he participated as a tutor, trainer, and member of the course planning group in the Bachelor Health Sciences and Master Human Movement Sciences. Thijs followed additional courses to improve his researcher skills. He presented his work at several national and international conferences. Furthermore, he is a lecturer at the Adelante Academy, he trains interdisciplinary rehabilitation teams skills in practicing exposure in vivo treatment. Thijs is married to Iverna and they live together with their daughter Lize. 


\section{Peer reviewed publications}

van Meulenbroek, T., Huijnen, I.P.J., Simons, L.E., Conijn, A.E.A., Engelbert, R.H.H. \& Verbunt, J.A. (2020). Exploring the underlying mechanism of pain-related disability in hypermobile adolescents with chronic musculoskeletal pain. Scandinavian Journal of Pain. doi: 10.1515/sjpain-2020-0023

Baadjou, V.A., den Hollander, M., van Meulenbroek, T., Verbunt, J.A. \& Timmers, I. (2020). Clinicians' initial experiences of transition to online interdisciplinary pain rehabilitation during the Covid-19 pandemic. Journal of Rehabilitation Medicine - Clinical Communications. doi: 10.2340/20030711-1000036

van Meulenbroek, T., Conijn, A.E.A., Huijnen, I.P.J., Engelbert, R.H.H. \& Verbunt, J.A. (2020). Multidisciplinary treatment for hypermobile adolescents with chronic musculoskeletal pain. Journal of Rehabilitation Medicine - Clinical Communications. doi: 10.2340/20030711-1000033

Dekker, C., van Haastregt, J.C.M., Verbunt, J.A., de Jong, J.R., van Meulenbroek, T., Pernot, H.F.M., van Velzen, A.D., Bastiaenen, C.H.G. \& Goossens, M.E.J.B. (2020). Painrelated fear in adolescents with chronic musculoskeletal pain: process evaluation of an interdisciplinary graded exposure program. BMC Health Services Research 2020. doi: 10.1186/s12913-020-505306

van Meulenbroek, T., Huijnen, I.P.J., Stappers, N.E.H., Engelbert, R.H.H. \& Verbunt, J.A. (2020). Generalized joint hypermobility and perceived harmfulness in healthy adolescents; impact on muscle strength, motor performance and physical activity level. Physiotherapy Theory and Practice. doi 10.1080/09593985.2019.1709231

van Laake-Geelen, C.C.M., Smeets, R.J.E.M., van Meulenbroek, T., den Hollander, M., Goossens, M.E.J.B. \& Verbunt, J.A. (2019). Exposure in vivo in patients with Painful Diabetic Neuropathy: Development of a treatment protocol. Journal of Rehabilitation Medicine - Clinical Communications. doi: 10.2340/20030711-1000015

van Meulenbroek, T., Huijnen, I.P.J, Wiertz, C.M.H. \& Verbunt, J.A. (2017). Pain-related fear and its disabling effect in hypermobile adolescents with chronic musculoskeletal pain. Journal of Orthopaedic \& Sports Physical Therapy. doi: 10.2519/jospt.2017.7282

van Meulenbroek, T., Huijnen, I.P.J., Meys, G.H.H. \& Rommers, G.M. (2017). Observatie van lopen in de huisartsenspreekkamer. Nederlands Tijdschrift Geneeskunde. 


\section{Submitted manuscripts}

van Meulenbroek, T., Huijnen, I.P.J., Engelbert, R.H.H. \& Verbunt, J.A. (submitted). Are chronic musculoskeletal pain and generalized joint hypermobility: disabling contributors to physical functioning?

den Hollander, M., Smeets, R.J.E.M., van Meulenbroek, T., van Laake-Geelen, C.C.M., Baadjou, V.A. \& Timmers, I. (submitted). Exposure in vivo as a treatment approach to target pain-related fear: theory and new insight from research and clinical practice.

\section{Other publications}

van Meulenbroek, T. (2019). PRISM: een kort screeningsinstrument om risico-ouders van jongeren met chronische pijn te identificeren. Nederlandstalig Tijdschrift Pijnbestrijding.

\section{Book chapters}

Spek, E.M., van Menxel, D.A.J., van Meulenbroek, T. \& Verbunt, J.A. (2017). Intake revalidatiearts en screening. In: Graded Exposure: Een cognitief gedragsmatige aanpak van chronische pijn. Uitgeverij Bohn Stafleu van Loghum, red: Verbunt, J.A. \& Smeets, R.J.E.M., pp. 97-105.

Spek, E.M., van Menxel, D.A.J., Ummels, B.J.A.G, van Meulenbroek, T. \& Verbunt, J.A. (2017). Graded Exposure in de praktijk: de behandeling bij jongeren. In: Graded Exposure: Een cognitief gedragsmatige aanpak van chronische pijn. Uitgeverij Bohn Stafleu van Loghum. red: Verbunt, J.A. \& Smeets, R.J.E.M., pp. 123-133. 


\section{Presentations at (inter)national scientific meetings}

June 2019: International Symposium on Pediatric Pain, Basel, Switzerland. Oral presentation "2B-Active: treating pain-related fear in adolescents and parents with chronic pain" in workshop "It takes two to tango: Evaluating the role of parents in psychological interventions for pediatric chronic pain", i.c.w. dr. I. Timmers, dr. A. Stone, and dr. R. Wicksell.

Oktober 2018: Symposium "Exposure bij chronische pijn: do or dare?", Maastricht, Nederland. Workshop "Het gebruik van de PHODA-youth".

Mei 2018: Landelijke scholingsbijeenkomst Onderwijs(zorg)consulenten, Utrecht, Nederland. Presentatie "Chronische pijn/vermoeidheid bij jongeren".

April 2018: Symposium "Chronische pijn bij kinderen, hoe nu verder?" Kinderpijncentrum Radboudumc, Nijmegen, Nederland. Presentatie "Exposure in vivo voor pijn aan het bewegingsapparaat".

November 2017: Symposium "Chronische pijn en vermoeidheid binnen de kinderrevalidatie" Heliomare, Wijk aan Zee, Nederland. Workshop "Het gebruik van de PHODA-youth in de behandeling van adolescenten met chronische pijn". 

